\title{
NIC-X鿉
}

NUCLEI IN T

schoo

ol

Debrecen

iviryaly

30
7-11 July 2014

www.nic2014.org

Nuclear reactions in astrophysics

Pierre Descouvemont

Université Libre de Bruxelles

Brussels, Belgium 


\section{Content of the lectures}

1. Introduction

1. Reaction networks

2. Needs for astrophysics

3. Specificities of nuclear astrophysics

2. Low-energy cross sections

1. Definitions

2. General properties

3. S-factor

3. Reaction rates

1. Definitions

2. Gamow peak

3. Resonant and non-resonant rates

4. General scattering theory (simple case: spins 0 , no charge, single channel)

1. Different models

2. Optical model

3. Scattering amplitude and cross sections

4. Phase-shift method

5. Resonances

6. Generalizations: Coulomb interaction, absorption, non-zero spins 
5. Models used in nuclear astrophysics

1. Brief overview

2. The potential model : Radiative-capture reactions

3. The R-matrix method

4. Microscopic models 
1. Introduction 


\section{Introduction}

Goal of nuclear astrophysics: understand the abundances of the elements

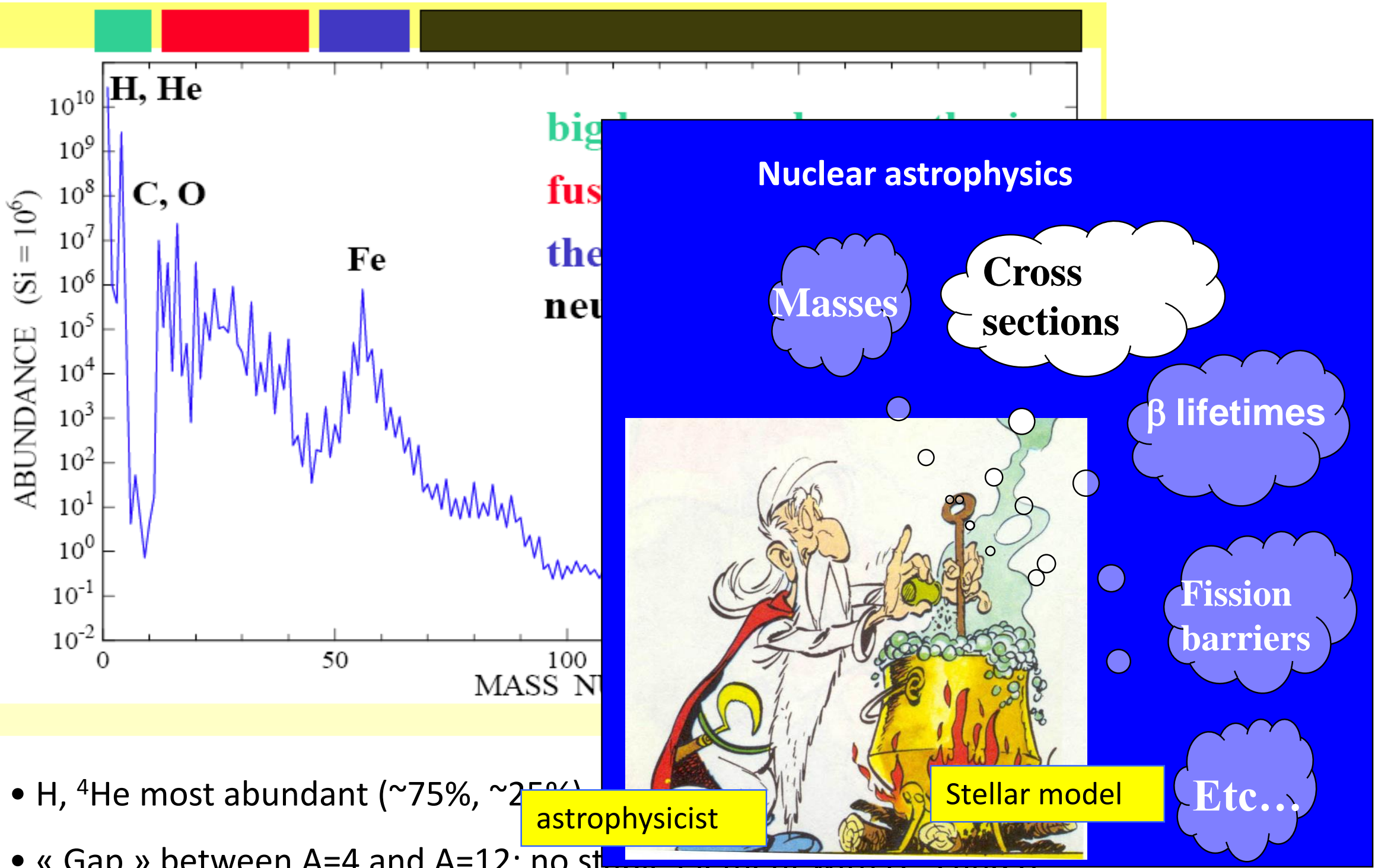

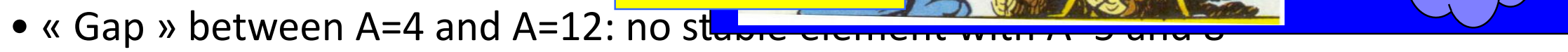

- Iron peak (very stable) 


\section{Introduction}

- Years 1940-50: Hoyle, Gamow

Role of nuclear reactions in stars

- Energy production

- Nucleosynthesis (Hoyle state in ${ }^{12} \mathrm{C}$ )

- 1957: B 2 FH: Burbidge, Burbidge, Fowler, Hoyle (Rev. Mod. Phys. 29 (1957) 547) Wikipedia site: http://en.wikipedia.org/wiki/B\%C2\%B2FH

Cycles: pp chain: converts $4 p \rightarrow{ }^{4} \mathrm{He}$

CNO cycle: converts $4 p \rightarrow{ }^{4} \mathrm{He}\left(\right.$ via ${ }^{12} \mathrm{C}$ )

s (slow) process: $(n, \gamma)$ capture followed by $\beta$ decay

$r$ (rapid) process: several $(n, \gamma)$ captures

$p$ (proton) process: $(p, \gamma)$ capture

- Nucleosynthesis:

Primordial (Bigbang): 3 first minutes of the Universe Stellar: star evolution, energy production

- Essentially two (experimental) problems in nuclear astrophysics

Low energies $\rightarrow$ very low cross sections (Coulomb barrier)

Need for radioactive beams

$\rightarrow$ in most cases a theoretical support is necessary (data extrapolation) 


\section{Introduction}

Reaction networks: set of equations with abundances of nucleus $\mathrm{m}: Y_{m}$

$$
\begin{aligned}
\frac{d Y_{m}}{d t}= & -\lambda_{m} Y_{m} & \rightarrow \text { Destruction of } \mathrm{m} \text { by } \beta \text { decay: } \lambda_{\mathrm{m}}=1 / \tau_{\mathrm{m}} \\
& +\sum_{k} \lambda_{k}^{(m)} Y_{k} & \rightarrow \text { Production of } \mathrm{m} \text { by } \beta \text { decay from elements } \mathrm{k} \\
& -\sum_{k} Y_{m} Y_{k}[m k]^{(m+k)} & \rightarrow \text { Destruction of } \mathrm{m} \text { by reaction with } \mathrm{k} \\
& +\sum_{k, l} Y_{k} Y_{l}[k l]^{(m)} & \rightarrow \text { Production of } \mathrm{m} \text { by reaction } \mathrm{k}+\mathrm{l} \rightarrow \mathrm{m}
\end{aligned}
$$

with $[\mathrm{kl}]^{(\mathrm{m}) \sim}\langle\sigma \mathrm{V}\rangle,\langle\sigma \mathrm{V}\rangle=$ reaction rate (strongly depends on temperature)

In practice:

- Many reactions are involved (no systematics)

- $\sigma$ must be known at very low energies $\rightarrow$ very low cross sections

- Reactions with radioactive elements are needed

- At high temperatures: high level densities $\rightarrow$ properties of many resonances needed 


\section{Introduction}

\section{Specificities of nuclear astrophysics}

- low energies (far below the Coulomb barrier)

$\rightarrow$ small cross sections

(in general not accessible in laboratories at stellar energies)

$\rightarrow$ low angular momenta (selection of resonances)

- radioactive nuclei

$\rightarrow$ need for radioactive beams $\left({ }^{7} \mathrm{Be},{ }^{13} \mathrm{~N},{ }^{18} \mathrm{~F}, \ldots\right)$

- different types of reactions:

- transfer $(\alpha, n),(\alpha, p),(p, \alpha)$, etc...

- radiative-capture: $(p, \gamma),(\alpha, \gamma),(n, \gamma)$, etc...

- weak processes: $p+p \rightarrow d+e^{+}+v$

- fusion: ${ }^{12} \mathrm{C}+{ }^{12} \mathrm{C},{ }^{16} \mathrm{O}+{ }^{16} \mathrm{O}$, etc.

- different situations

- capture, transfer

- resonant, non resonant

- low level density (light nuclei), high level density (heavy nuclei)

- peripheral, internal processes

$\rightarrow$ different approaches, for theory and for experiment 


\section{Introduction}

Some key reactions

- $\mathrm{d}(\alpha, \gamma)^{6} \mathrm{Li},{ }^{3} \mathrm{He}(\alpha, \gamma)^{7} \mathrm{Be}$ : Big-Bang

- Triple $\alpha,{ }^{12} \mathrm{C}(\alpha, \gamma){ }^{16} \mathrm{O}$ : He burning

- ${ }^{7} \mathrm{Be}(p, \gamma)^{8} \mathrm{~B}$ : solar neutrino problems

- ${ }^{18} F(p, \alpha){ }^{15} O$ : nova nucleosynthesis

- Etc... 


\section{Low-energy cross sections}

- Definitions

- General properties

- S-factor 


\section{Low-energy cross sections}

Types of reactions: general definitions valid for all models

\begin{tabular}{|l|l|l|}
\hline Type & Example & Origin \\
\hline Transfer & ${ }^{3} \mathrm{He}\left({ }^{3} \mathrm{He}, 2 \mathrm{p}\right) \alpha$ & Strong \\
\hline Radiative capture & ${ }^{2} \mathrm{H}(\mathrm{p}, \gamma)^{3} \mathrm{He}$ & Electromagnetic \\
\hline Weak capture & $\mathrm{p}+\mathrm{p} \rightarrow \mathrm{d}+\mathrm{e}^{+}+\mathrm{v}$ & Weak \\
\hline
\end{tabular}

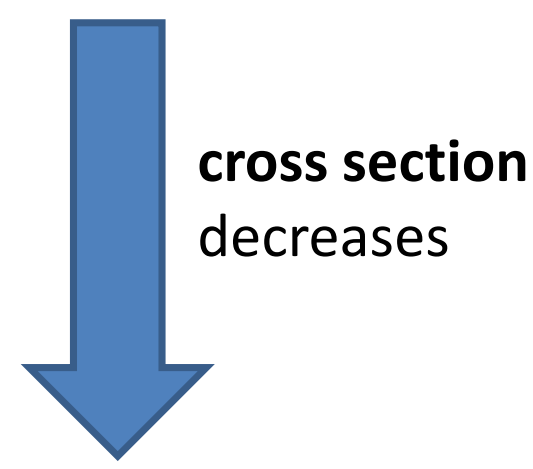




\section{Low-energy cross sections}

- Transfer: $\mathrm{A}+\mathrm{B} \rightarrow \mathrm{C}+\mathrm{D}\left(\sigma_{\mathrm{t}}\right.$, strong interaction, example: $\left.{ }^{3} \mathrm{He}(\mathrm{d}, \mathrm{p})^{4} \mathrm{He}\right)$

$$
\sigma_{t, c \rightarrow c^{\prime}}(E)=\frac{\pi}{k^{2}} \sum_{J \pi} \frac{2 J+1}{\left(2 I_{1}+1\right)\left(2 I_{2}+1\right)}\left|U_{c c^{\prime}}^{J \pi}(E)\right|^{2}
$$

$U_{c c^{\prime}}^{J \pi}(E)=$ collision (scattering) matrix (obtained from scattering theory $\rightarrow$ various models) $c, c^{\prime}=$ entrance and exit channels

Transfer reaction:

Nucleons are transfered
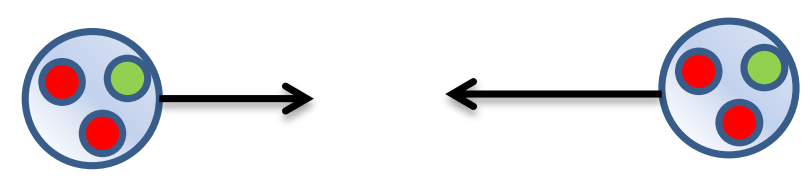
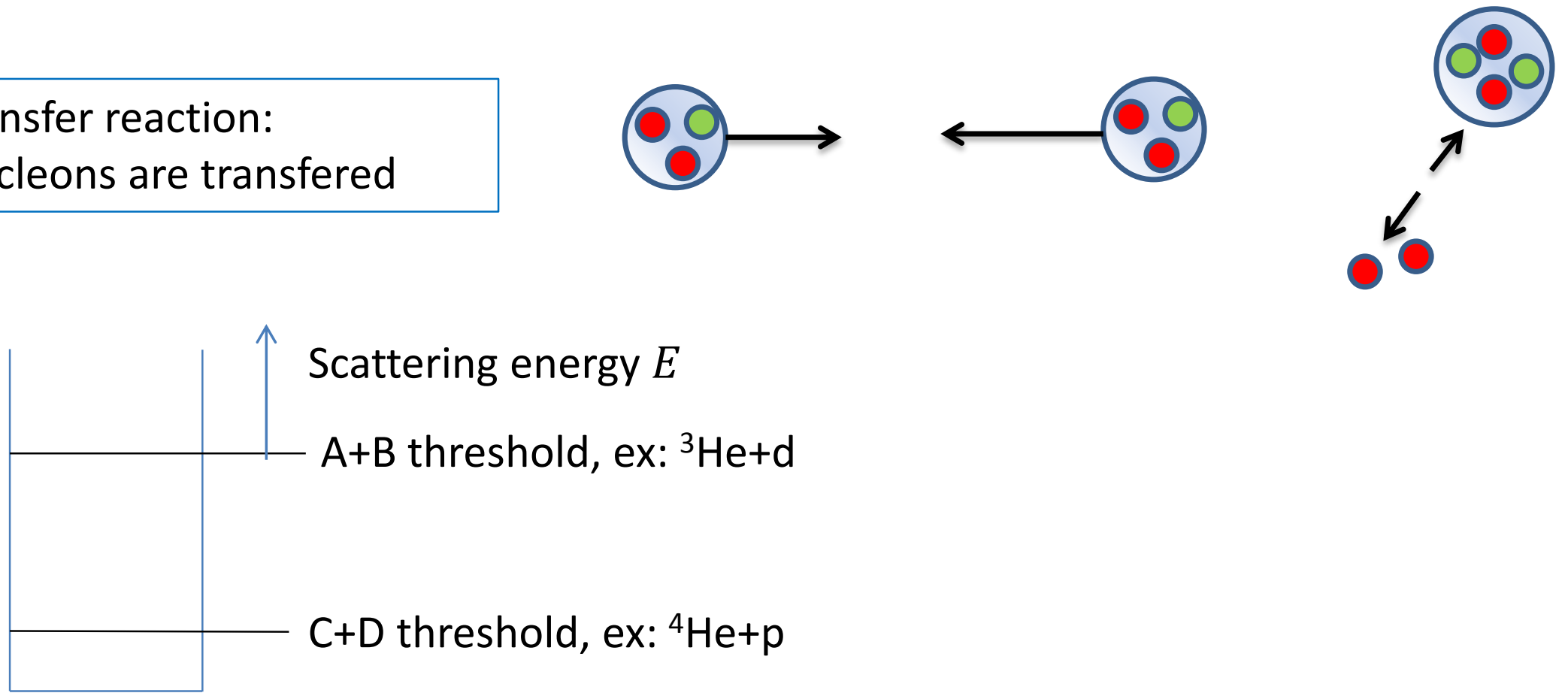

Compound nucleus, ex: ${ }^{5} \mathrm{Li}$ 


\section{Low-energy cross sections}

- Radiative capture : $\mathrm{A}+\mathrm{B} \rightarrow \mathrm{C}+\gamma\left(\sigma_{\mathrm{C}}\right.$, electromagnetic interaction, example: $\left.{ }^{12} \mathrm{C}(\mathrm{p}, \gamma)^{13} \mathrm{~N}\right)$

$$
\sigma_{C}^{J_{f} \pi_{f}}(E) \sim \sum_{\lambda} \sum_{J_{i} \pi_{i}} k_{\gamma}^{2 \lambda+1}\left|<\Psi^{J_{f} \pi_{f}}\left\|\mathcal{M}_{\lambda}\right\| \Psi^{J_{i} \pi_{i}}(E)>\right|^{2}
$$

$J_{f} \pi_{f}=$ final state of the compound nucleus $\mathrm{C}$ $\Psi J_{i} \pi_{i}(E)=$ initial scattering state of the system (A+B)

$\mathcal{M}_{\lambda \mu}=$ electromagnetic operator (electric or magnetic): $\mathcal{M}_{\lambda \mu} \sim \operatorname{er} r^{\lambda} Y_{\lambda}^{\mu}\left(\Omega_{r}\right)$

Capture reaction:

A photon is emitted
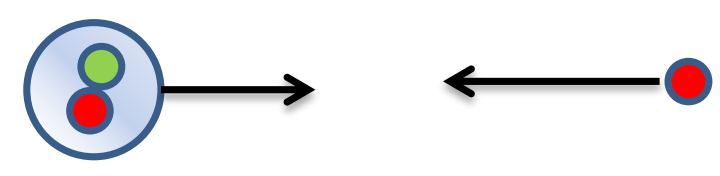

Long wavelength approximation:

Wave number $k_{\gamma}=E_{\gamma} / \hbar c$, wavelength: $\lambda_{\gamma}=2 \pi / k_{\gamma}$

Typical value: $E_{\gamma}=1 \mathrm{MeV}, \lambda_{\gamma} \approx 1200 \mathrm{fm}>>$ typical dimensions of the system $(R)$

$\rightarrow k_{\gamma} R \ll 1=$ Long wavelength approximation 


\section{Low-energy cross sections}

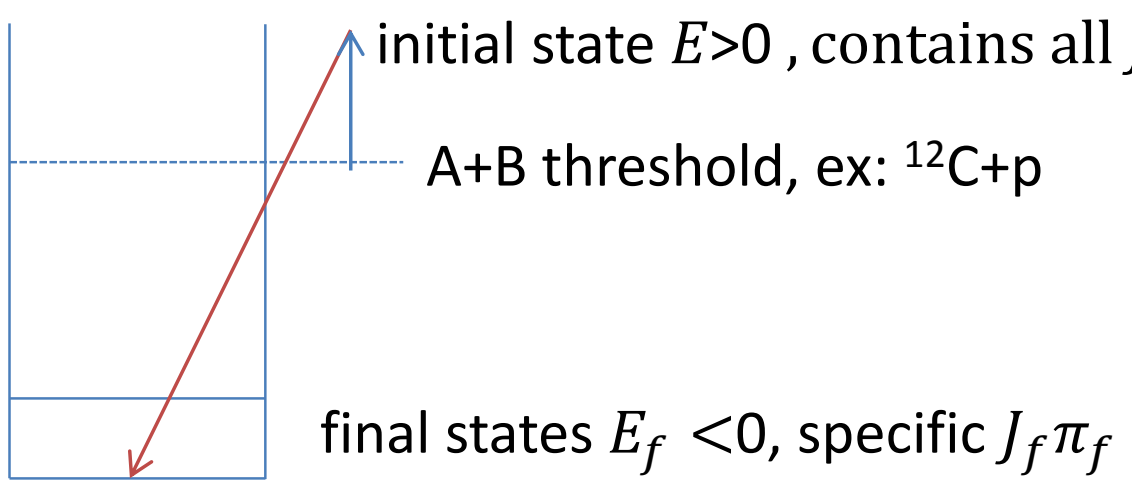

$$
\sigma_{C}^{J_{f} \pi_{f}}(E) \sim \sum_{J_{i} \pi_{i}} \sum_{\lambda} k_{\gamma}^{2 \lambda+1}\left|<\Psi^{J_{f} \pi_{f}}\left\|\mathcal{M}_{\lambda}\right\| \Psi J_{i} \pi_{i}(E)>\right|^{2}
$$

- $k_{\gamma}=\left(E-E_{f}\right) / \hbar c=$ photon wave number

- In practice

- Summation over $\lambda$ limited to 1 term (often E1, or E2/M1 if E1 is forbidden)

$\frac{E 2}{E 1} \sim\left(k_{\gamma} R\right)^{2} \ll 1$ (from the long wavelength approximation)

- Summation over $J_{i} \pi_{i}$ limited by selection rules

$$
\begin{aligned}
& \left|J_{i}-J_{f}\right| \leq \lambda \leq J_{i}+J_{f} \\
& \pi_{i} \pi_{f}=(-1)^{\lambda} \text { for electric, } \pi_{i} \pi_{f}=(-1)^{\lambda+1} \text { for magnetic }
\end{aligned}
$$




\section{Low-energy cross sections}

Example 1: ${ }^{8} \mathrm{Be}(\alpha, \gamma)^{12} \mathrm{C}$

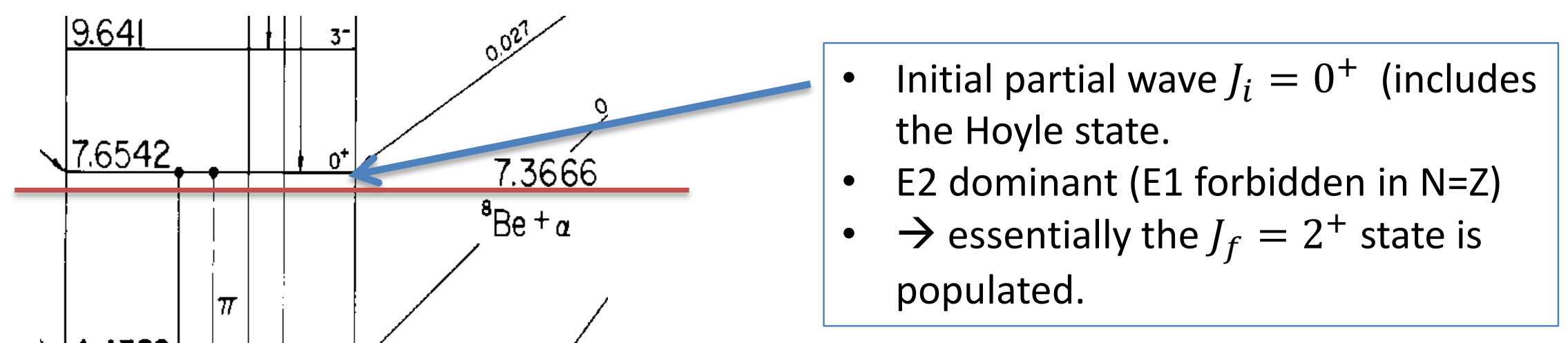




\section{Low-energy cross sections}

\section{Example $2:{ }^{14} \mathrm{~N}(\mathrm{p}, \gamma)^{15} \mathrm{O}$}

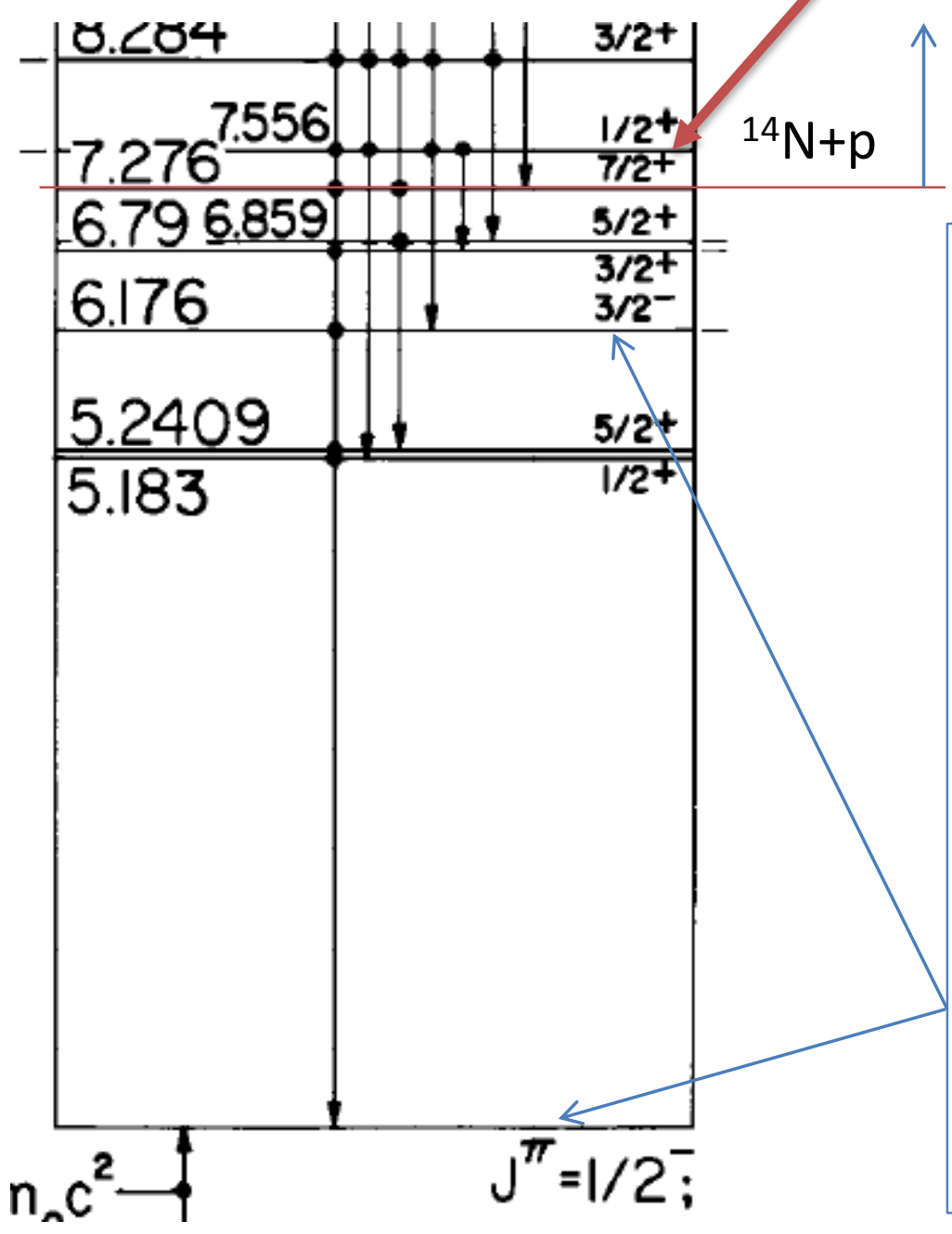

$\mathrm{E}$ (all $J_{i}$ values)

- Spin of ${ }^{14} \mathrm{~N}: I_{1}=1^{+}$, proton $I_{2}=1 / 2^{+}$

- Channel spin $I$ :

$$
\rightarrow I=1 / 2,3 / 2
$$

- Orbital momentum $\ell$

$$
|I-\ell| \leq J_{i} \leq I+\ell
$$

- At low energies, $\ell=0$ is dominant $\rightarrow J_{i}=$ $1 / 2^{+}, 3 / 2^{+}$

- multipolarity E1 $\rightarrow$ transitions to $J_{f}=$ $1 / 2^{-}, 3 / 2^{-}, 5 / 2^{-}$

- Resonance $1 / 2^{+}$determines the cross section 


\section{Low-energy cross sections}

Example $3:{ }^{12} \mathrm{C}(\alpha, \gamma){ }^{16} \mathrm{O},{ }^{15} \mathrm{~N}(\mathrm{p}, \gamma){ }^{16} \mathrm{O},{ }^{15} \mathrm{~N}(\mathrm{p}, \alpha){ }^{12} \mathrm{C}$

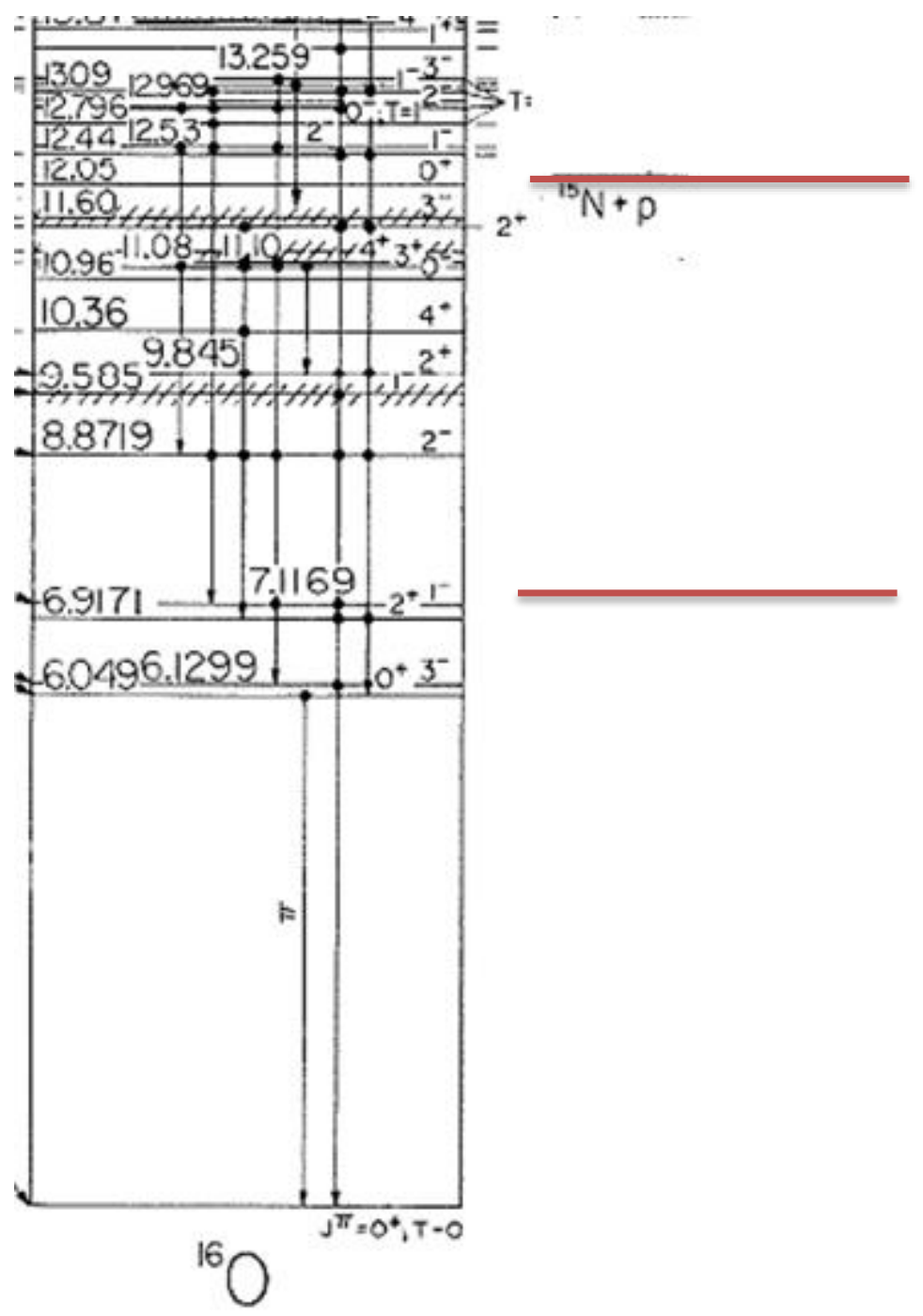

${ }^{15} \mathrm{~N}+\mathrm{p}$ threshold

${ }^{15} \mathrm{~N}(p, \gamma){ }^{16} \mathrm{O}$ and ${ }^{15} \mathrm{~N}(\mathrm{p}, \alpha){ }^{12} \mathrm{C}$ are open

$\rightarrow{ }^{15} \mathrm{~N}(p, \gamma)^{16} \mathrm{O}$ negligible

${ }^{12} \mathrm{C}+\alpha$ threshold

only possibility: ${ }^{12} \mathrm{C}(\alpha, \gamma){ }^{16} \mathrm{O}$

$\rightarrow{ }^{12} \mathrm{C}(\alpha, \gamma)^{16} \mathrm{O}$ (very) important 


\section{Low-energy cross sections}

- Weak capture $\left(p+p \rightarrow d+v+e^{-}\right)$: tiny cross section

$\rightarrow$ no measurement (only calculations)

$$
\sigma_{W}^{J_{f} \pi_{f}}(E) \sim \sum_{J_{i} \pi_{i}}\left|<\Psi^{J_{f} \pi_{f}}\left\|O_{\beta}\right\| \Psi^{J_{i} \pi_{i}}(E)>\right|^{2}
$$

- Calculations similar to radiative capture

○ $O_{\beta}=$ Fermi $\left(\sum_{i} t_{i \pm}\right)$ and Gamow-Teller $\left(\sum_{i} t_{i \pm} \sigma_{i}\right)$ operators

$\circ{ }^{3} \mathrm{He}+\mathrm{p} \rightarrow{ }^{4} \mathrm{He}+v+\mathrm{e}^{-}:$produces high-energy neutrinos (more than tiny!) 


\section{Low-energy cross sections}

- Fusion: similar to transfer, but with many output channels $\rightarrow$ statistical treatment

$\rightarrow$ optical potentials
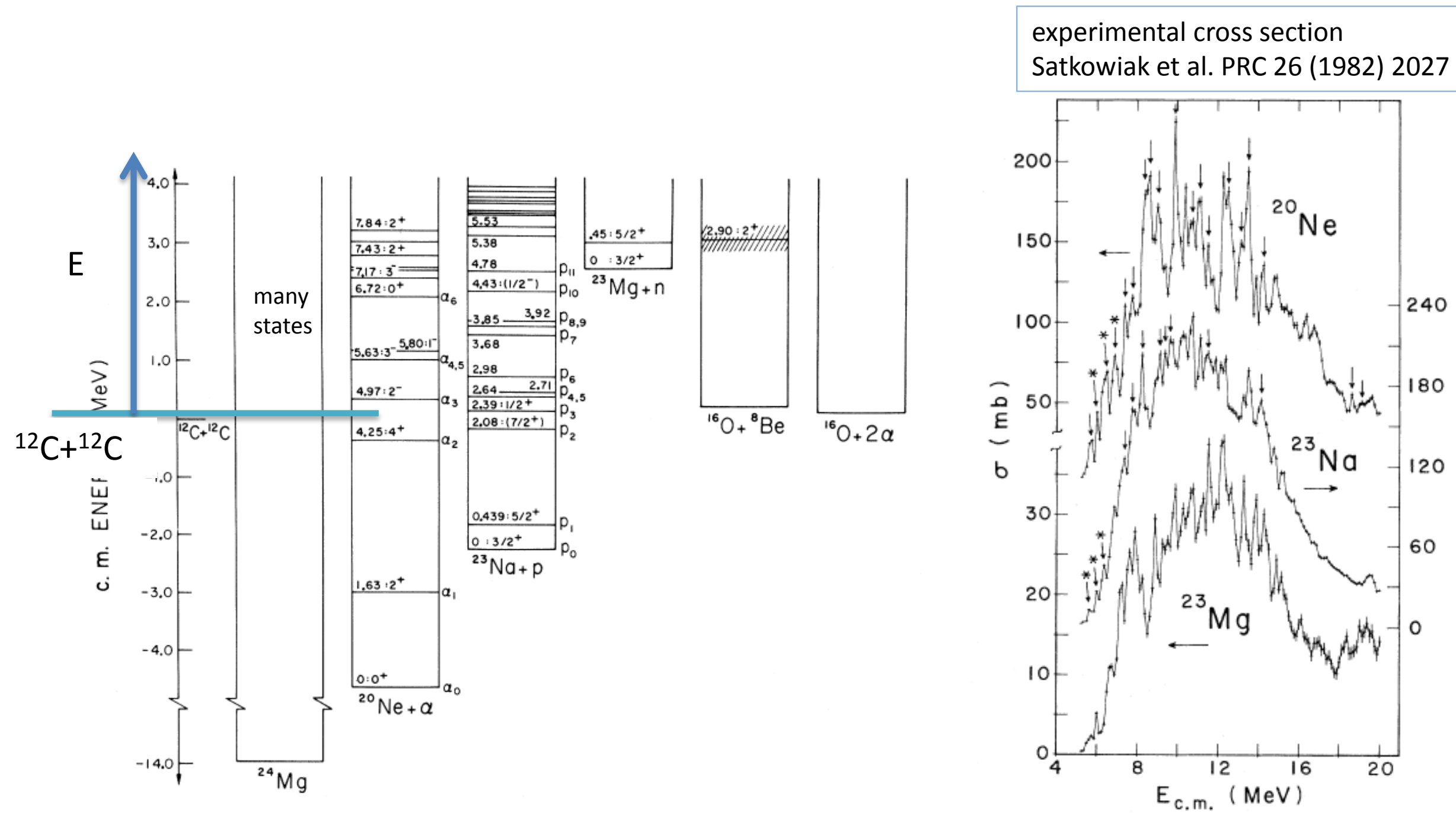


\section{Low-energy cross sections}

General properties (common to all reactions)

Scattering energy $\mathrm{E}: \quad$ wave function $\Psi_{i}(E)$ common to all processes

Reaction threshold

$\mathrm{V}(\mathrm{r})$

$\ell>0$

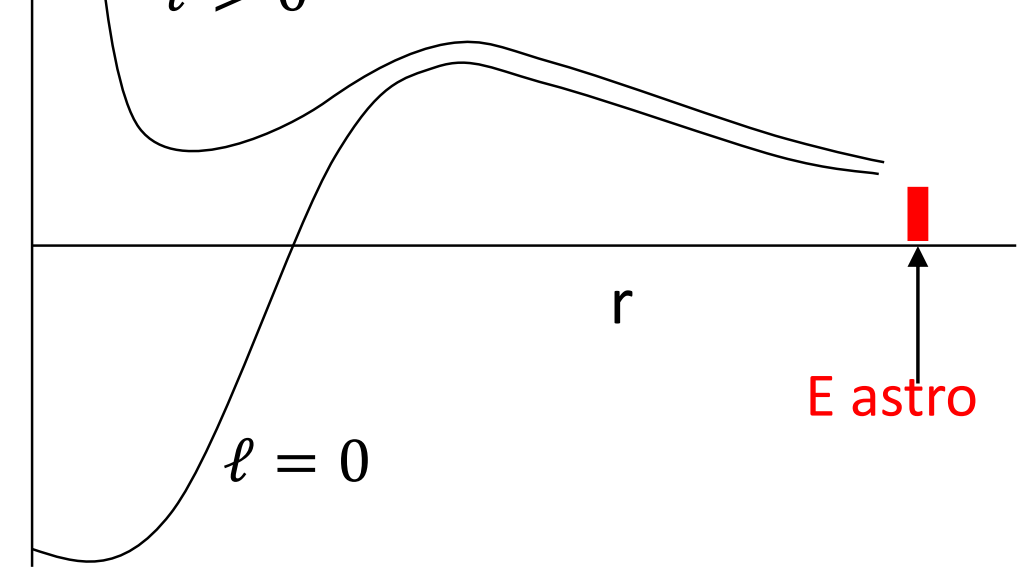

- Cross sections dominated by Coulomb effects Sommerfeld parameter $\eta=Z_{1} Z_{2} e^{2} / \hbar v$

- Coulomb functions at low energies

$$
\begin{aligned}
& F_{\ell}(\eta, x) \rightarrow \exp (-\pi \eta) \mathcal{F}_{\ell}(x), \\
& G_{\ell}(\eta, x) \rightarrow \exp (\pi \eta) \mathcal{G}_{\ell}(x),
\end{aligned}
$$

- Coulomb effect: strong $E$ dependence : $\exp (2 \pi \eta)$ neutrons: $\sigma(E) \sim 1 / v$

- Strong $\ell$ dependence

Centrifugal term: $\sim \frac{\hbar^{2}}{2 \mu} \frac{\ell(\ell+1)}{r^{2}}$

$\rightarrow$ stronger for nucleons $(\mu \approx 1)$ than for $\alpha(\mu \approx 4)$ 


\section{Low-energy cross sections}

General properties: specificities of the entrance channel $\rightarrow$ common to all reactions

- All cross sections (capture, transfer) involve a summation over $\ell: \sigma(E)=\sum_{\ell} \sigma_{\ell}(E)$

- The partial cross sections $\sigma_{\ell}(E)$ are proportional to the penetration factor

$$
P_{\ell}(E)=\frac{k a}{F_{\ell}(k a)^{2}+G_{\ell}(k a)^{2}}(a=\text { typical radius })
$$

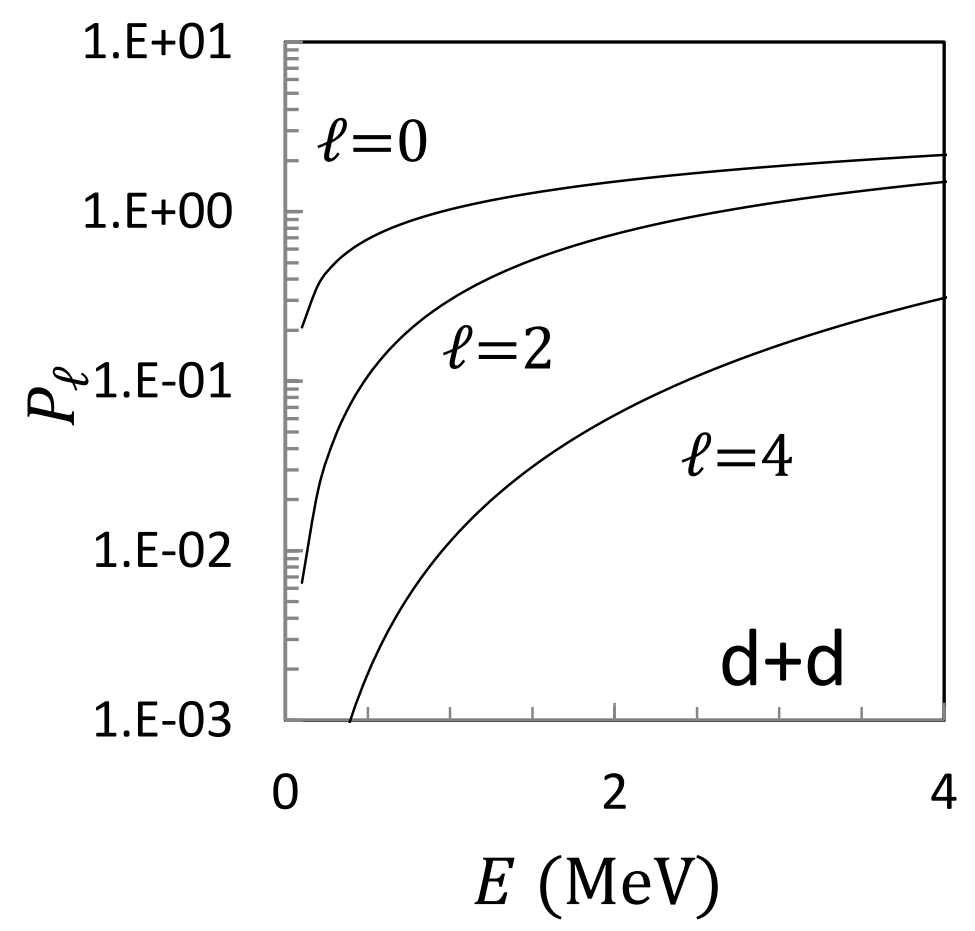

\section{Consequences}

- $\ell>0$ are often negligible at low energies

- $\ell=\ell_{\text {min }}$ is dominant (often $\ell_{\text {min }}=0$ )

- For $\ell=0, P_{0}(E) \sim \exp (-2 \pi \eta)$

Astrophysical S factor: $S(E)=\sigma(E) E \exp (2 \pi \eta)$ (Units: $\mathrm{E}^{*} \mathrm{~L}^{2}: \mathrm{MeV}$-barn)

- removes the coulomb dependence $\rightarrow$ only nuclear effects

- weakly depends on energy $\rightarrow \sigma(E) \approx S_{0} \exp (-2 \pi \eta) / E$ (any reaction at low $\mathrm{E}$ ) 


\section{Low-energy cross sections}

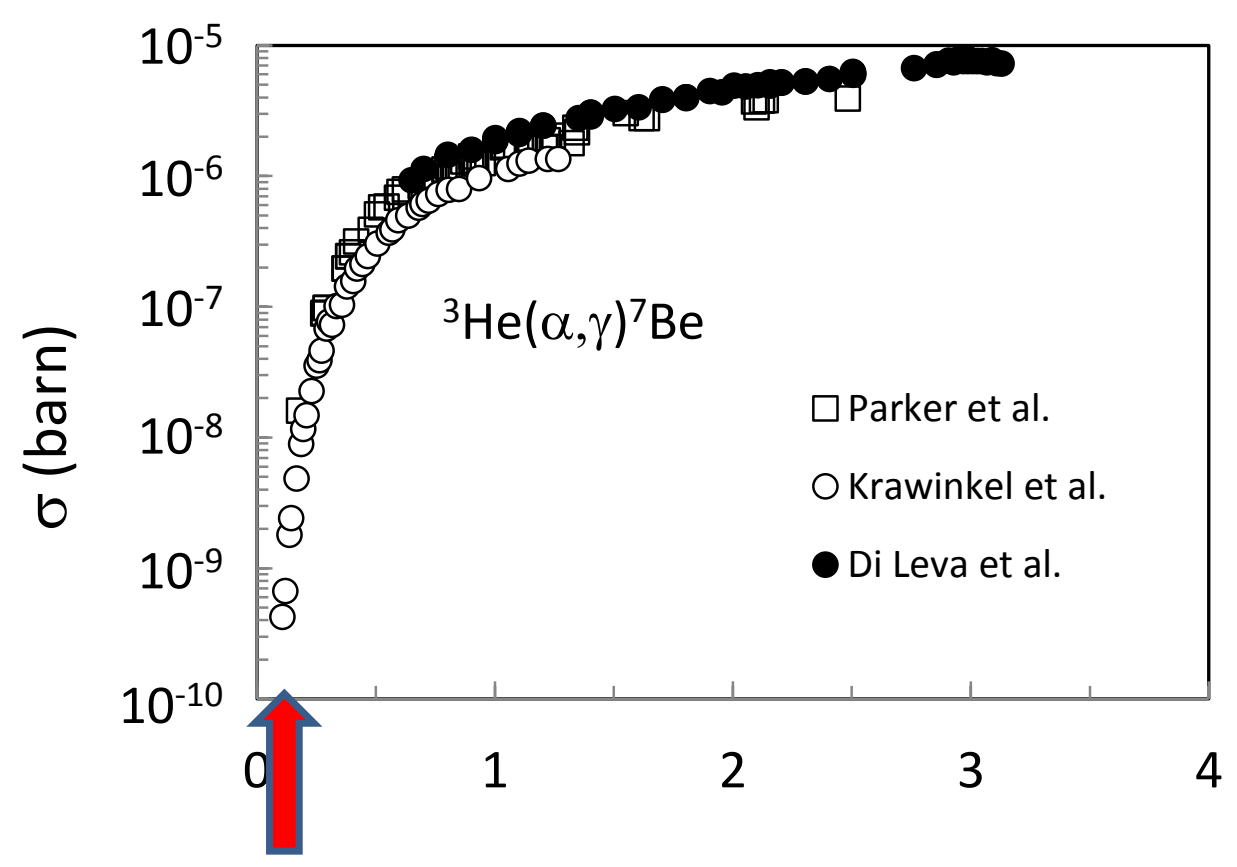

$$
\text { non resonant:S(E) }=\sigma(E) E \exp (2 \pi \eta)
$$

Example: ${ }^{3} \mathrm{He}(\alpha, \gamma)^{7} \mathrm{Be}$ reaction

- Cross section $\sigma(E)$ Strongly depends on energy

- Logarithmic scale

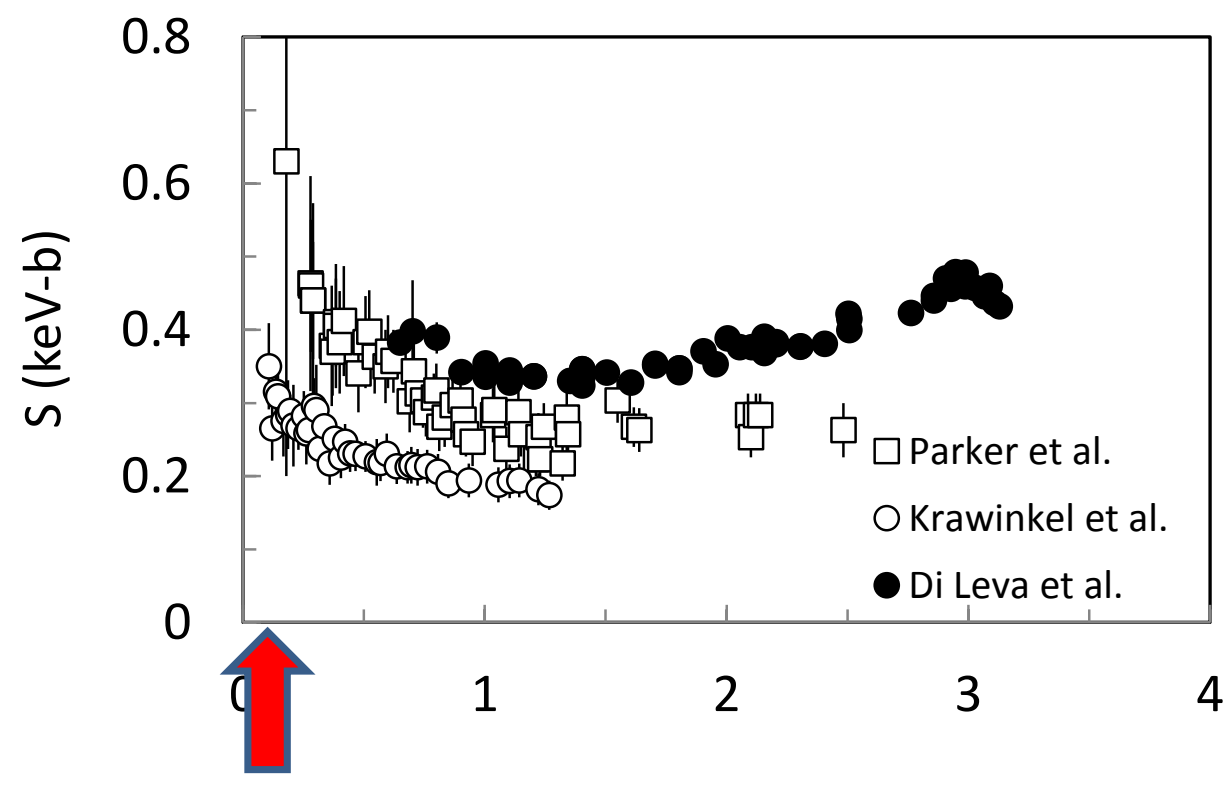

S factor

- Coulomb effects removed

- Weak energy dependence

- Linear scale 


\section{Low-energy cross sections}

Resonant cross sections: Breit-Wigner form

$$
\sigma_{R}(E) \approx \frac{\pi}{k^{2}} \frac{\left(2 J_{R}+1\right)}{\left(2 I_{1}+1\right)\left(2 I_{2}+1\right)} \frac{\Gamma_{1}(E) \Gamma_{2}(E)}{\left(E_{R}-E\right)^{2}+\Gamma^{2} / 4}
$$

- $J_{R}, E_{R}=$ spin, energy of the resonance

- Valid for any process (capture, transfer)

- Valid for a single resonance $\rightarrow$ several resonances need to be added (if necessary)

- $\Gamma_{1}=$ Partial width in the entrance channel (strongly depends on $E, \ell$ )

$\Gamma_{1}(E)=2 \gamma_{1}^{2} P_{\ell}(E)$ with $\gamma_{1}^{2}=$ reduced width (does not depend on $E$ )

$$
P_{\ell}(E) \sim \exp (-2 \pi \eta)
$$

A resonance at low energies is always narrow (role of $P_{\ell}(E)$ )

- $\Gamma_{2}=$ Partial width in the exit channel (weakly depends on $E, \ell$ )

○ Transfer: $\Gamma_{2}(E)=2 \gamma_{2}^{2} P_{\ell_{f}}(E+Q) \quad$ (in general $Q \gg E \rightarrow P_{\ell_{f}}(E+Q)$ almost constant)

○ Capture: $\Gamma_{2}(E) \sim\left(E-E_{f}\right)^{2 \lambda+1} B(E \lambda) \rightarrow$ weak energy dependence

- S factor near a resonance $S(E)=\sigma(E) E \exp (2 \pi \eta)$

$$
S_{R}(E) \sim \frac{\gamma_{1}^{2} \Gamma_{2}}{\left(E_{R}-E\right)^{2}+\Gamma^{2} / 4} P_{\ell}(E) \exp (2 \pi \eta) \text { Almost constant }
$$

$\rightarrow$ Simple estimate at low $\mathrm{E}$ (at the Breit-Wigner approximation) 


\section{Low-energy cross sections}

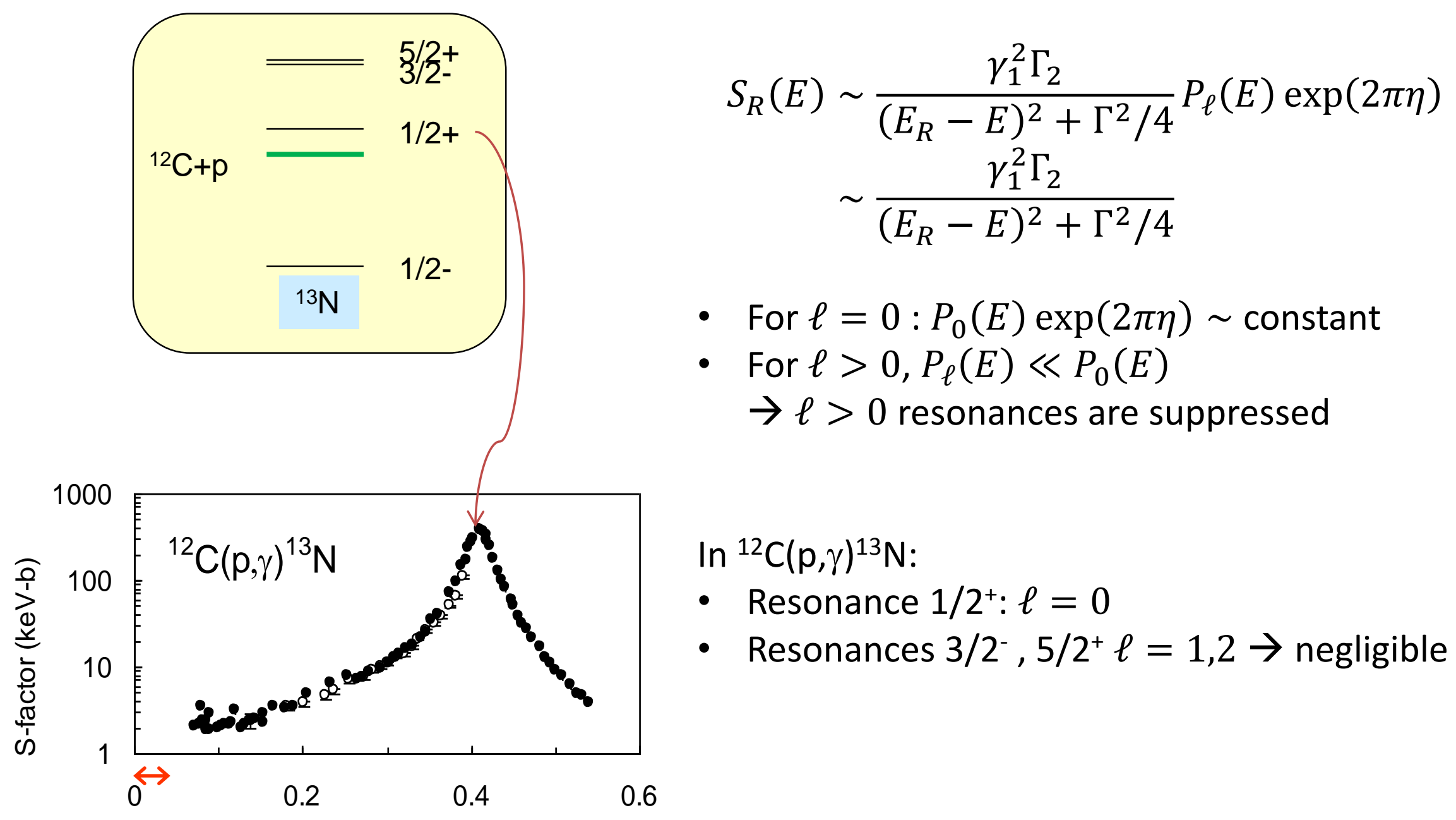

Note: BW is an approximation

- Neglects background, external capture

- Assumes an isolated resonance

- Is more accurate near the resonance energy 


\section{Low-energy cross sections}

${ }^{3} \mathrm{He}(\mathrm{d}, \mathrm{p})^{4} \mathrm{He}$ : isolated resonance in a transfer reaction

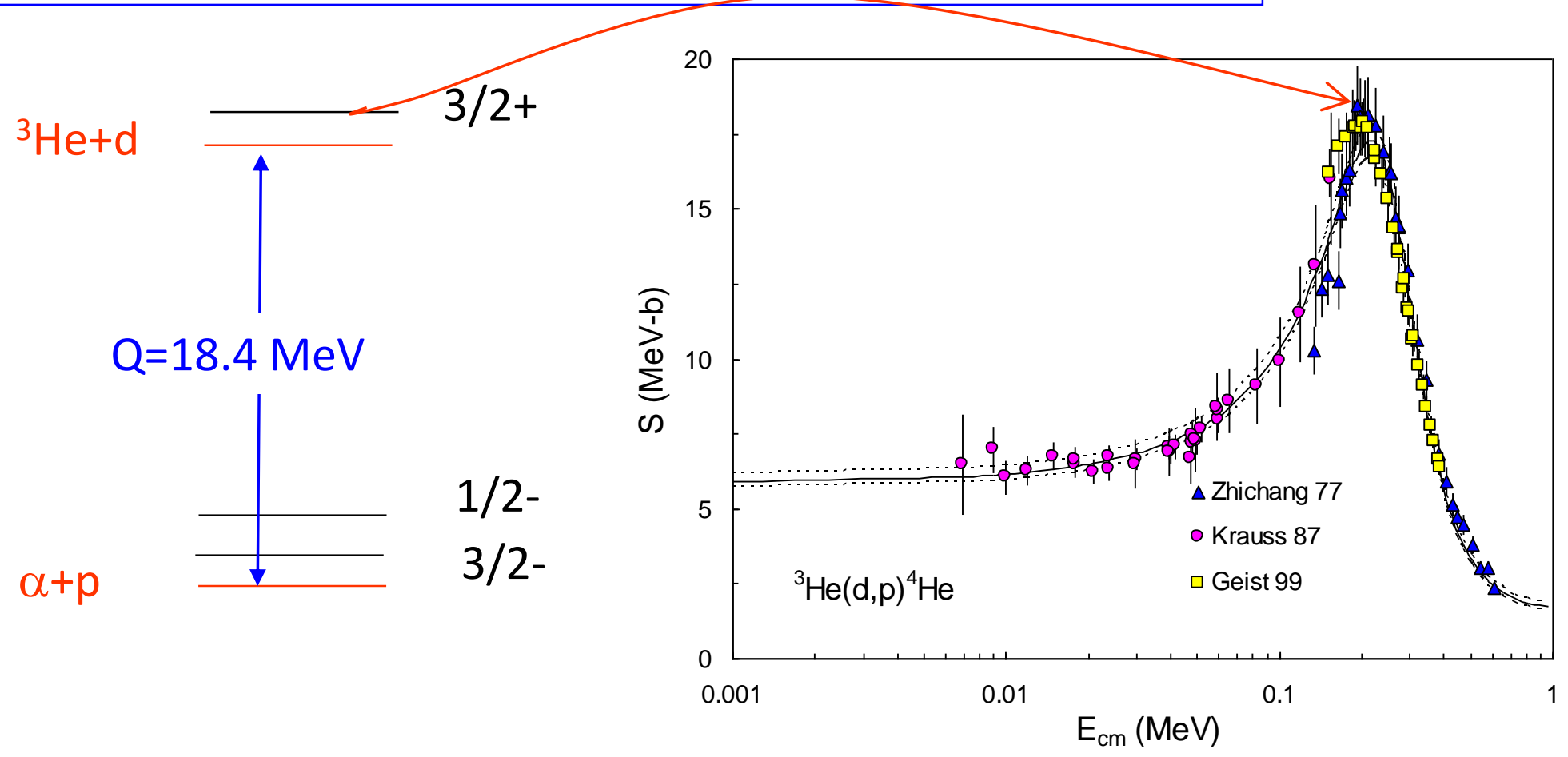

$3 / 2+$ resonance:

- Entrance channel: spin $\mathrm{S}=1 / 2,3 / 2$, parity $+\rightarrow \ell=0,2$

- Exit channel: $\operatorname{spin} S=1 / 2$, parity $+\rightarrow \ell=1$ 


\section{Low-energy cross sections}

Breit Wigner approximation

$$
\sigma_{d p}(E) \approx \frac{\pi}{k^{2}} \frac{\left(2 J_{R}+1\right)}{\left(2 I_{1}+1\right)\left(2 I_{2}+1\right)} \frac{\Gamma_{d}(E) \Gamma_{p}(E)}{\left(E_{R}-E\right)^{2}+\Gamma^{2} / 4}
$$

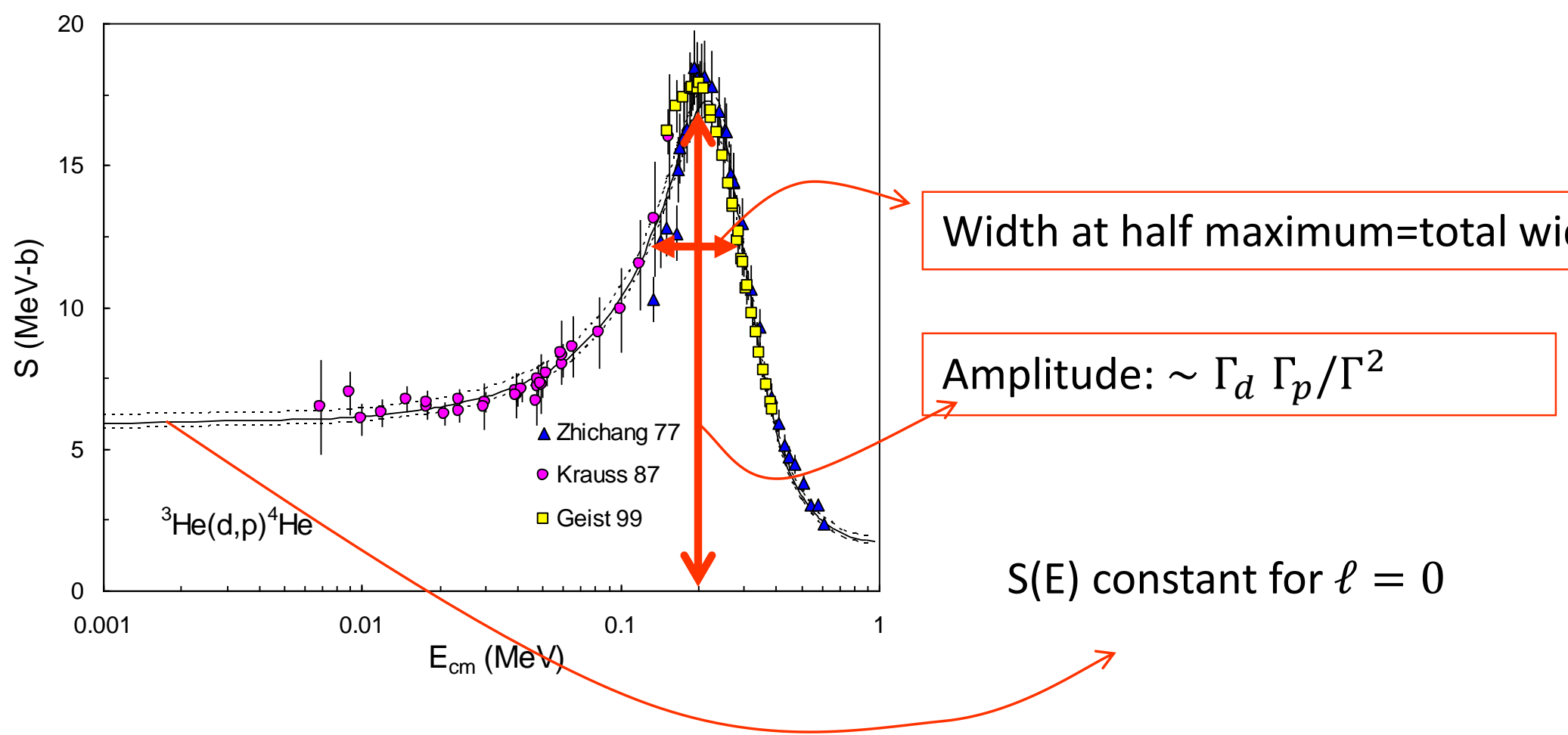




\section{Low-energy cross sections}

Two comments: 1 . Selection of the main resonances

2. Going beyond the Breit-Wigner approximation

1. Selection of the main resonances

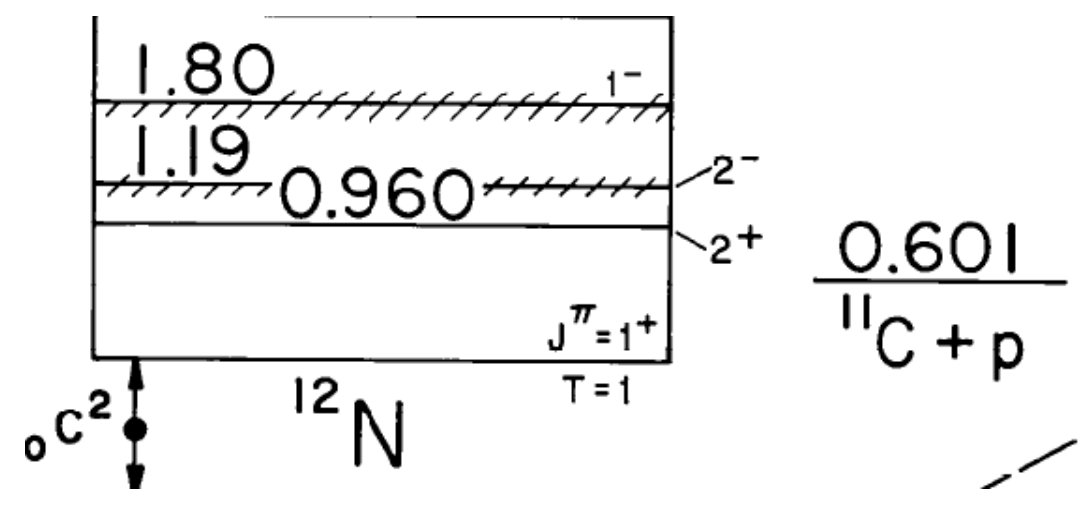

$\left.{ }^{11} \mathrm{C}(p, \gamma)\right)^{12} \mathrm{~N}\left(\operatorname{spin}{ }^{11} \mathrm{C}=3 / 2^{-}\right)$

- Resonance $2: \ell=0, \mathrm{E} 1$

- Resonance $2^{+}: \ell=1, \mathrm{E} 2 / \mathrm{M} 1$ $\rightarrow$ negligible

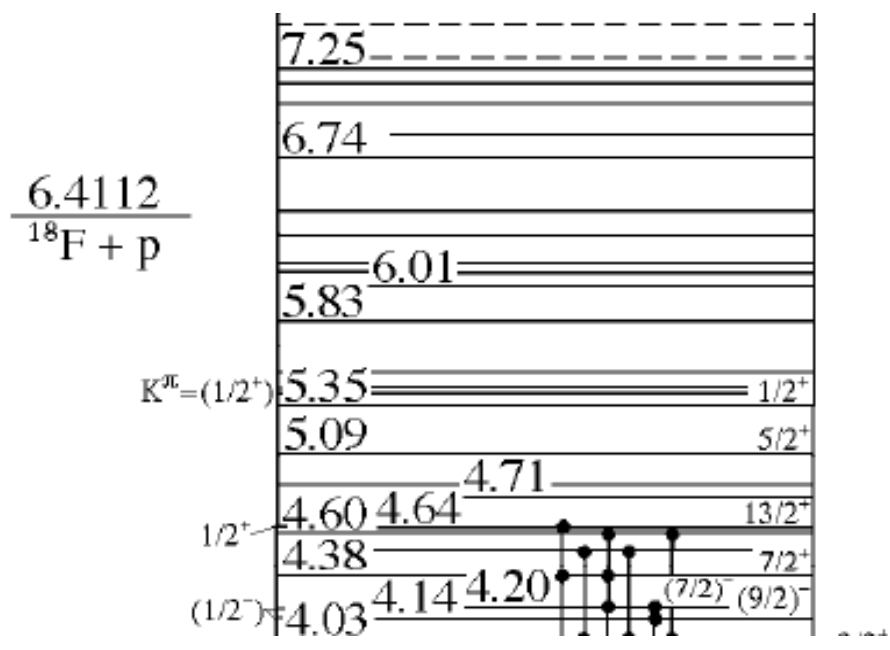

${ }^{18} \mathrm{~F}(\mathrm{p}, \alpha){ }^{15} \mathrm{O} \quad\left(\operatorname{spin}{ }^{18} \mathrm{~F}=1^{+}\right)$

- Many resonances

- Only $\ell=0$ resonances are important $\rightarrow J=1 / 2^{+}, 3 / 2^{+}$only

$\rightarrow$ In general a small number of resonances play a role 


\section{Low-energy cross sections}

2. Going beyond the Breit-Wigner approximation

- How to go beyond the BW approximation?

- Problem of vocabulary

- Direct capture

- External capture

- Non-resonant capture = « direct » capture

$\rightarrow$ confusion!

- External capture $\sigma(E)=\left|M_{\text {int }}+M_{\text {ext }}\right|^{2}$

With $\quad \sigma_{B W}(E)=\left|M_{\text {int }}\right|^{2}$

$M_{\text {ext }} \sim C$, with C=Asymptotic Normalization Constant (ANC) is needed

- $\quad$ Non resonant capture : $\sigma(E)=\sum_{\ell} \sigma_{\ell}(E)=\sigma_{R}(E)+\sum_{\ell \neq \ell_{R}} \sigma_{\ell}(E)$

$\rightarrow$ scanning the resonance is necessary 


\section{Low-energy cross sections}

Many different situations

- Transfer cross sections (strong interaction)

- Non resonant:

- Resonant, with $\ell_{\mathrm{R}}=\ell_{\min }$ :

- Resonant, with $\ell_{R}>\ell_{\min }$ :

- Multiresonance:
${ }^{6} \mathrm{Li}(p, \alpha)^{3} \mathrm{He}$

${ }^{3} \mathrm{He}(\mathrm{d}, \mathrm{p}) \alpha$

${ }^{11} \mathrm{~B}(p, \alpha)^{8} \mathrm{Be}$

${ }^{22} \mathrm{Ne}(\alpha, \mathrm{n})^{25} \mathrm{Mg}$

- Capture cross sections (electromagnetic interaction)

- Non resonant:

- Resonant, with $\ell_{\mathrm{R}}=\ell_{\min }$ :

- Resonant, with $\ell_{R}>\ell_{\min }$ :

- Multiresonance:

- Subthreshold state:

- Weak capture cross sections (weak interaction.

- Non resonant
${ }^{6} \mathrm{Li}(p, \gamma)^{7} \mathrm{Be}$

${ }^{12} \mathrm{C}(\mathrm{p}, \gamma)^{13} \mathrm{~N}$

${ }^{7} \mathrm{Be}(\mathrm{n} v)^{8} \mathrm{~B}$

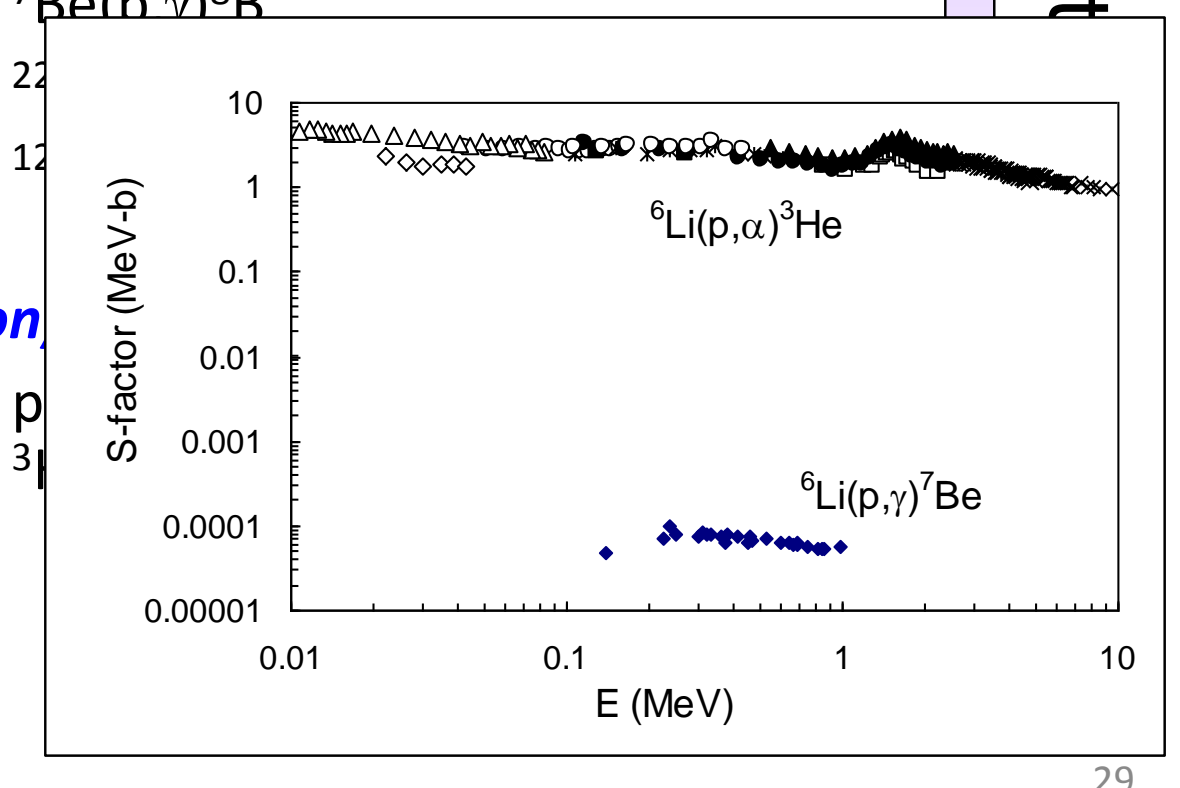




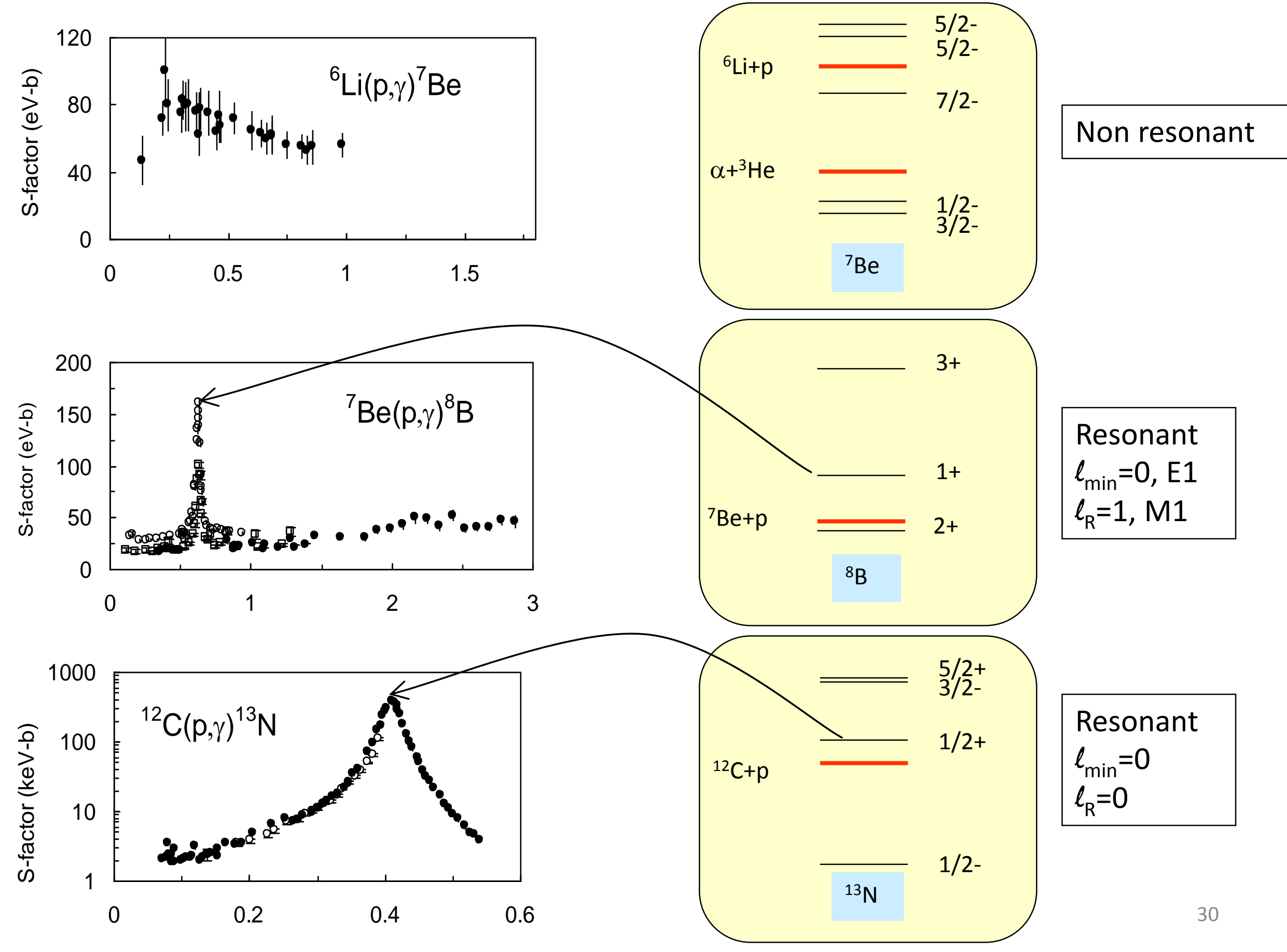


3. Reaction rates

1. Definitions

2. Gamow peak

3. Non-resonant rates

4. Resonant rates 


\section{Reaction rates}

\section{Definition}

Quantity used in astrophysics: reaction rate (integarl over the energy E)

$$
N_{A}<\sigma v>=N_{A} \int \sigma(E) v N(E, T) d E
$$

- Definition valid for resonant and non-resonant reactions

- $N_{A}=$ Avogadro number

- $T$ =temperature, $v=$ velocity, $k_{B}=$ Boltzmann constant $\left(k_{B} \sim \frac{1}{11.6} \mathrm{MeV} / 10^{9} \mathrm{~K}\right)$

- $N(E, T)=\left(\frac{8 E}{\pi \mu m_{N}\left(k_{B} T\right)^{3}}\right)^{1 / 2} \exp \left(-\frac{E}{k_{B} T}\right)=$ Maxwell-Boltzmann distribution

- $\frac{1}{N_{A}<\sigma v>}=$ typical reaction time

- 2 approaches

- numerical

- analytical: non-resonant and resonant reactions treated separately

$\rightarrow$ essentially two energy dependences: $\quad \exp \left(-\frac{E}{k_{B} T}\right):$ decreases with $\mathrm{E}$ $\exp (-2 \pi \eta)$ : increases with $\mathrm{E}$ 


\section{Reaction rates}

\section{The Gamow peak}

Defines the energy range relevant for the reaction rate (non-resonant reactions)

\section{Linear scale}

\section{Logarithmic scale}

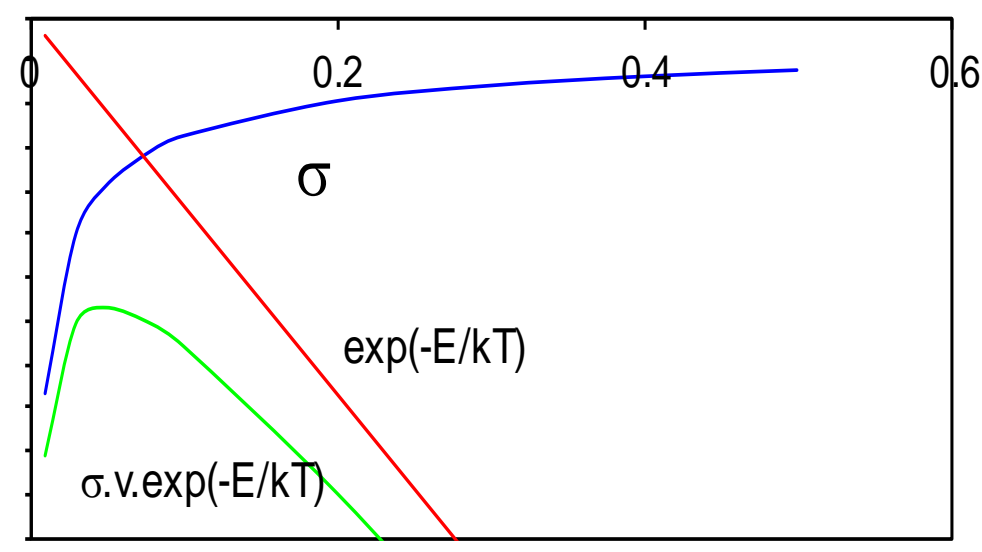

$\mathrm{Ecm}$
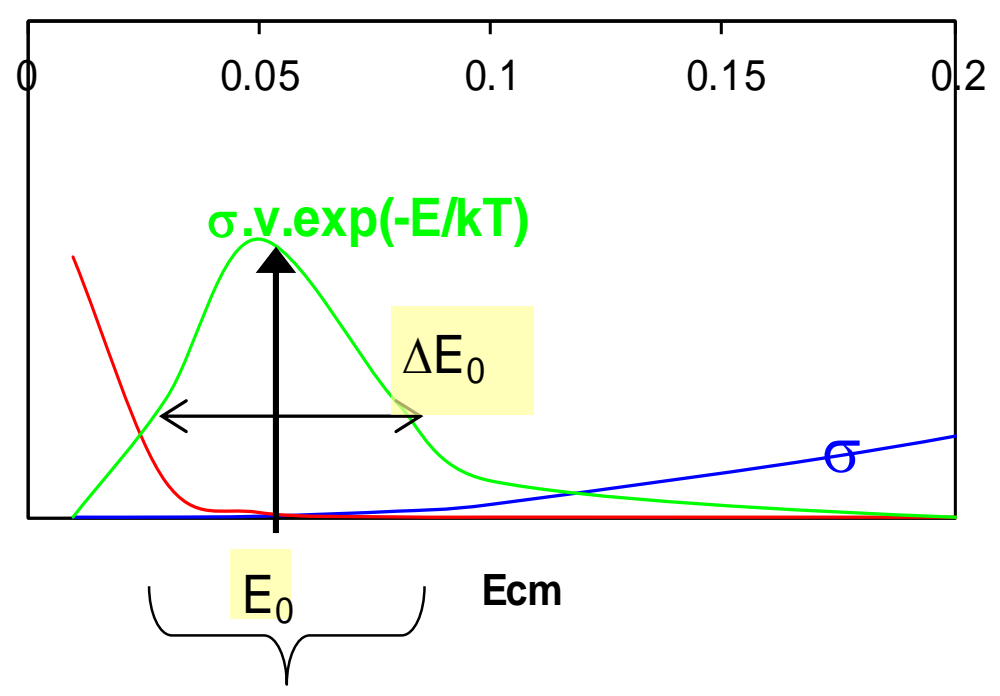

Gamow peak ( Gaussian, depends on T)

Gamow peak : $E_{0}=0.122 \mu^{1 / 3}\left(Z_{1} Z_{2} T_{9}\right)^{2 / 3} \mathrm{MeV}$ : lower than the Coulomb barrier increases with T

$$
\Delta E_{0}=0.237 \mu^{1 / 6}\left(Z_{1} Z_{2}\right)^{1 / 3} T_{9}^{5 / 6} \mathrm{MeV}
$$

$=$ Energy range where $\sigma(E)$ must be known $\left(T_{9}=T\right.$ in $\left.10^{9} \mathrm{~K}\right)$ 


\section{Reaction rates}

\section{Examples}

\begin{tabular}{|c|c|c|c|c|c|}
\hline Reaction & $\mathrm{T}\left(10^{9} \mathrm{~K}\right)$ & $\mathrm{E}_{0}(\mathrm{MeV})$ & $\Delta \mathrm{E}_{0}(\mathrm{MeV})$ & $\mathrm{E}_{\text {coul }}(\mathrm{MeV})$ & $\sigma\left(\mathrm{E}_{0}\right) / \sigma\left(\mathrm{E}_{\text {coul }}\right)$ \\
\hline$d+p$ & 0.015 & 0.006 & 0.007 & 0.3 & $10^{-4}$ \\
\hline${ }^{3} \mathrm{He}+{ }^{3} \mathrm{He}$ & 0.015 & 0.021 & 0.012 & 1.2 & $10^{-13}$ \\
\hline$\alpha+{ }^{12} \mathrm{C}$ & 0.2 & 0.3 & 0.17 & 3 & $10^{-11}$ \\
\hline${ }^{12} \mathrm{C}+{ }^{12} \mathrm{C}$ & 1 & 2.4 & 1.05 & 7 & $10^{-10}$ \\
\hline
\end{tabular}

$\square E_{0} / E_{\text {coul }} \approx 0.3 T_{9}^{2 / 3}(\mathrm{p}$ and $\alpha)$

$\square$ At low $T_{9}, E_{0} \ll E_{\text {coul }}$ (coulomb barrier)

V Very low cross sections at stellar temperatures (different for neutrons: no barrier)

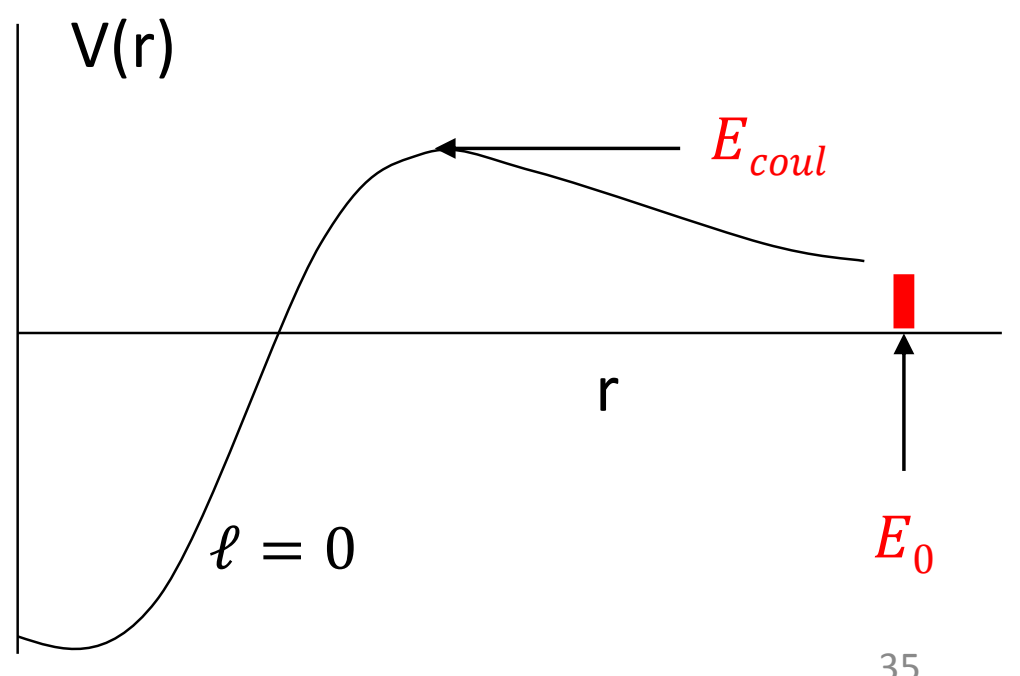




\section{Reaction rates}

\section{Non-resonant reaction rates}

- Approximation: Taylor expansion about the minimum $E=E_{0}: 2 \pi \eta+E / k_{B} T \approx c_{0}+\left(\frac{E-E_{0}}{2 \Delta E_{0}}\right)^{2}$

$$
\text { Then }\left\langle\sigma v>\approx\left(\frac{8}{\pi \mu m_{N}\left(k_{B} T\right)^{3}}\right)^{1 / 2} \exp \left(-3 \frac{E_{0}}{k_{B} T}\right) \int S(E) \exp \left(-\left(\frac{E-E_{0}}{2 \Delta E_{0}}\right)^{2}\right) d E\right.
$$

- $S(E)$ is assumed constant $\left(=S\left(E_{0}\right)\right)$ in the Gamow peak $\rightarrow\left\langle\sigma v>\sim S\left(E_{0}\right) \exp \left(-3 \frac{E_{0}}{k_{B} T}\right) / T^{2 / 3}\right.$, with $\mathrm{E}_{0}=0.122 \mu^{1 / 3}\left(Z_{1} Z_{2} T_{9}\right)^{2 / 3} \mathrm{MeV}$

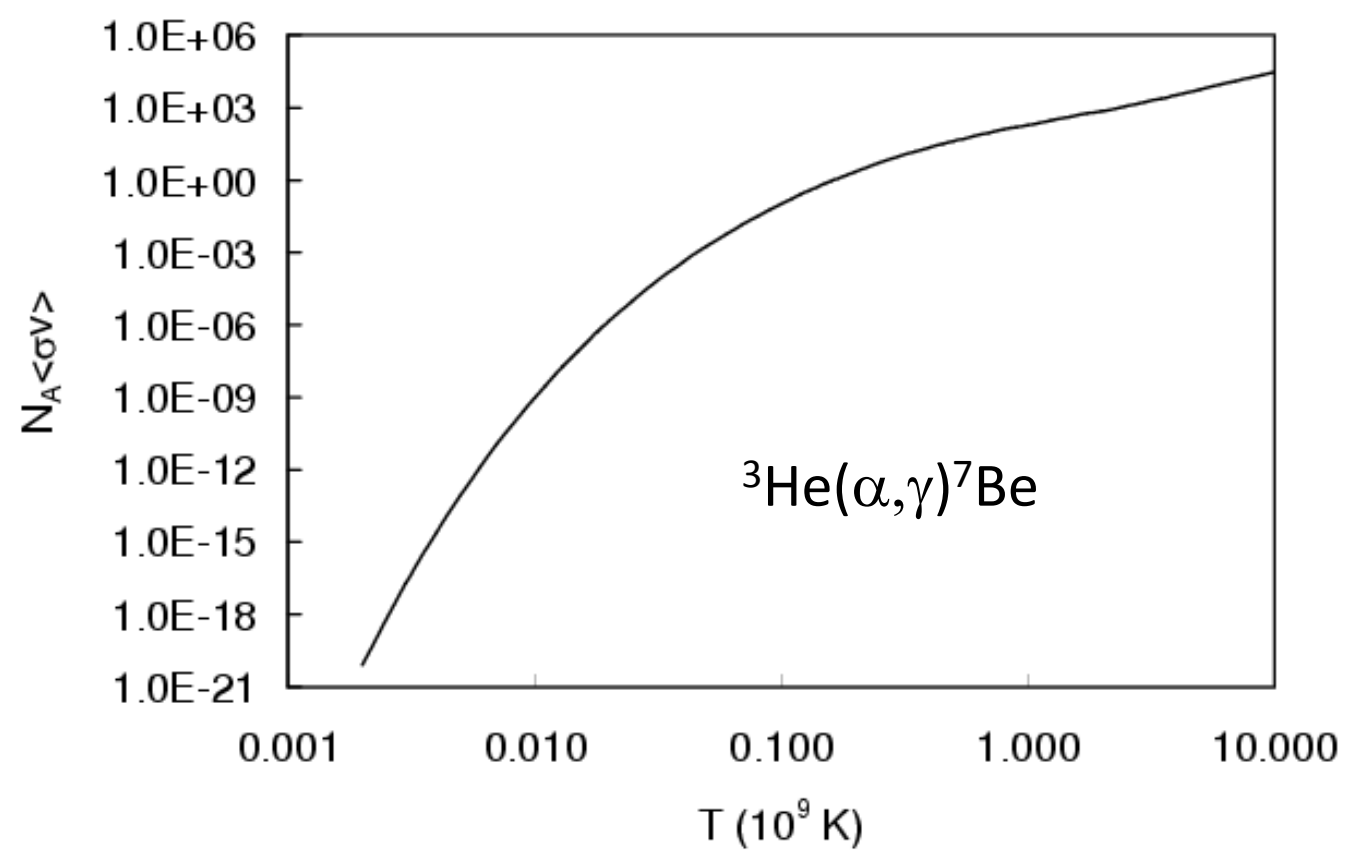

Very fast variation with the temperature 


\section{Reaction rates}

\section{Resonant reaction rates}

- General definition: $N_{A}\langle\sigma v\rangle=N_{A} \int \sigma(E) v N(E, T) d E$

here $\sigma(E)$ is given by the Breit-Wigner approximation

$$
\sigma(E) \approx \frac{\pi}{k^{2}} \frac{\left(2 J_{R}+1\right)}{\left(2 I_{1}+1\right)\left(2 I_{2}+1\right)} \frac{\Gamma_{1}(E) \Gamma_{2}(E)}{\left(E_{R}-E\right)^{2}+\Gamma^{2} / 4}
$$

- This provides

$$
\begin{aligned}
& <\sigma v>_{R}=\left(\frac{2 \pi}{\mu m_{N} k_{B} T}\right)^{3 / 2} \hbar^{2} \omega \gamma \exp \left(-\frac{E_{R}}{k_{B} T}\right) \\
& \omega \gamma=\frac{2 J_{R}+1}{\left(2 I_{1}+1\right)\left(2 I_{2}+1\right)} \frac{\Gamma_{1} \Gamma_{2}}{\Gamma_{1}+\Gamma_{2}}
\end{aligned}
$$

- $\omega \gamma=$ resonance " strength »

- $\Gamma_{1}, \Gamma_{2}=$ partial widths in the entrance and exit channels

- For a reaction $(p, \gamma): \Gamma_{\gamma}<<\Gamma_{p} \rightarrow \omega \gamma \sim \Gamma_{\gamma}$

- Valid for capture and transfer

- Rate strongly depends on the resonance enrgy

$\rightarrow$ In general: competition between resonant and non-resonant contributions 


\section{Reaction rates}

Tail contribution: for a given resonance

For a resonance: $\langle\sigma v\rangle \sim \int S(E) \exp \left(-2 \pi \eta-E / k_{B} T\right) d E$

- Non resonant: $S(E) \approx S_{0}: 1$ maximum at $E=E_{0}$

- Resonant: $S(E)=\mathrm{BW}: 2$ maxima at $E=E_{R}$ does not depend on $\mathrm{T}$

$E=E_{0}$ : depends on $\mathrm{T}$

$\rightarrow 2$ contributions to the rate : $N_{A}\langle\sigma v\rangle \approx N_{A}\langle\sigma v\rangle_{R}+N_{A}\langle\sigma v\rangle_{T}$

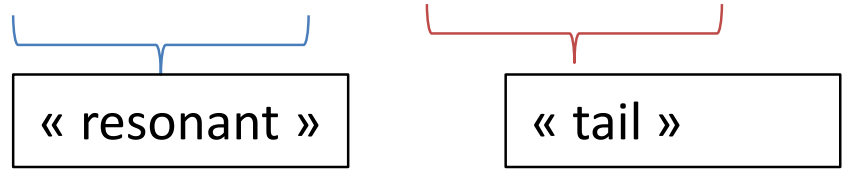

- $<\sigma v>_{R}=\left(\frac{2 \pi}{\mu m_{N} k_{B} T}\right)^{3 / 2} \hbar^{2} \omega \gamma \exp \left(-\frac{E_{R}}{k_{B} T}\right)$

- $<\sigma v>_{T} \sim S\left(E_{0}\right) \exp \left(-3 \frac{E_{0}}{k_{B} T}\right) / T^{2 / 3}$, with $S\left(E_{0}\right) \sim \frac{\Gamma_{1}\left(E_{0}\right) \Gamma_{2}\left(E_{0}\right)}{\left(E_{R}-E_{0}\right)^{2}+\Gamma^{2} / 4}$

O Both contributions depend on temperature: in most cases one term is dominant

o " Critical temperature »: when $E_{0}=E_{R} \rightarrow$ separation not valid 


\section{Reaction rates}

Example ${ }^{12} \mathrm{C}(\mathrm{p}, \gamma){ }^{13} \mathrm{~N}: E_{R}=0.42 \mathrm{MeV}$

Integrant $S(E) \exp \left(-2 \pi \eta-E / k_{B} T\right)$

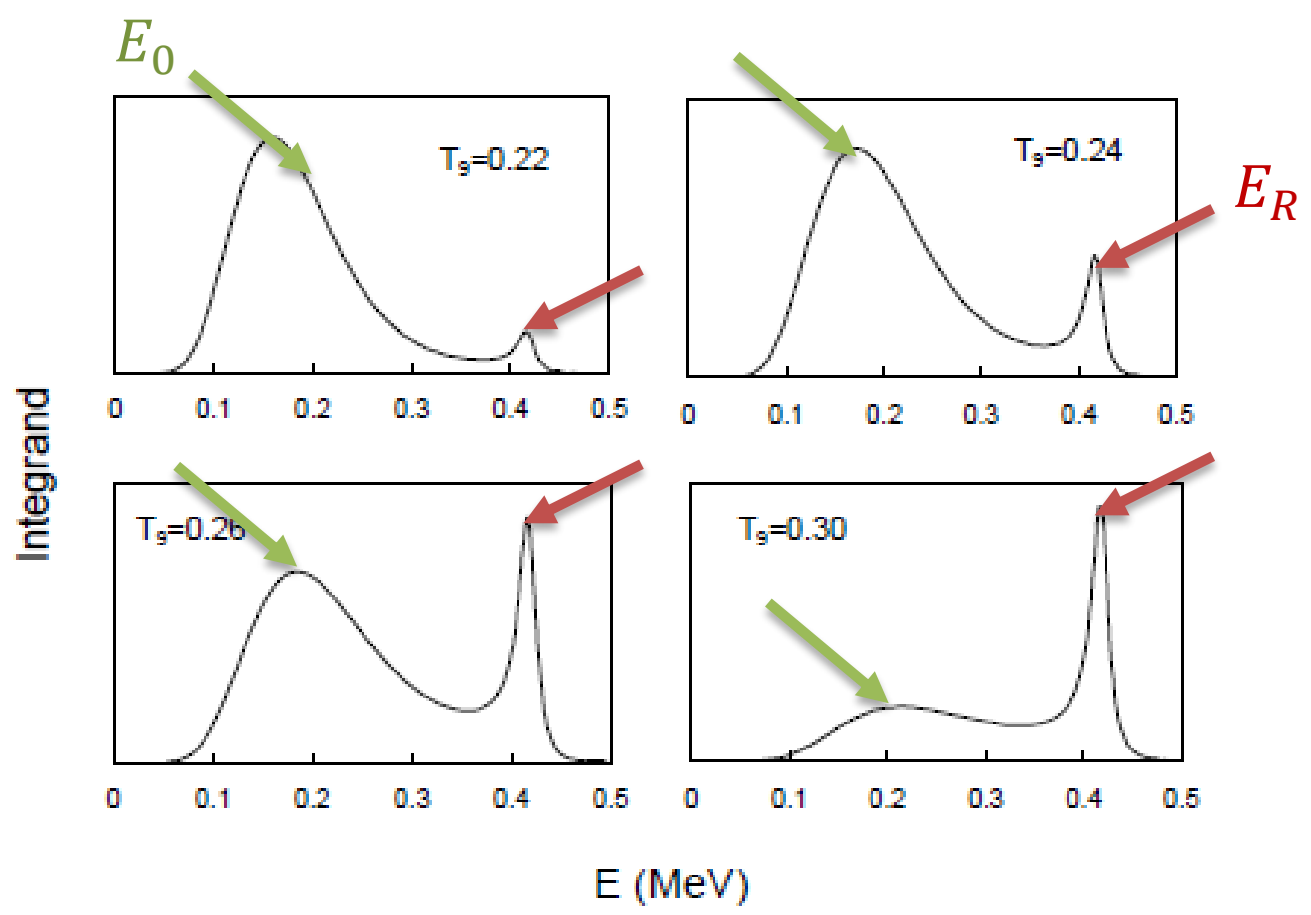

${ }^{12} \mathrm{C}(p, \gamma){ }^{13} \mathrm{~N}$

Above $T_{9} \approx 0.3: \quad$ "resonant " contribution is dominant

requires $E_{R}, \omega \gamma$ only (no individual partial widths)

strongly depends on $E_{R}: \exp \left(-E_{R} / k_{B} T\right)$

Below $T_{9} \approx 0.2: \quad E_{0} \ll E_{R}:$ « tail » contribution is dominant requires both widths

weakly depends on $E_{R}: 1 /\left(\left(E_{R}-E_{0}\right)^{2}+\Gamma^{2} / 4\right)$ 


\section{General scattering theory \\ 1. Different models \\ 2. Potential/optical model \\ 3. Scattering amplitude and cross section (elastic scattering)}




\section{General scattering theory}

Scheme of the collision (elastic scattering)

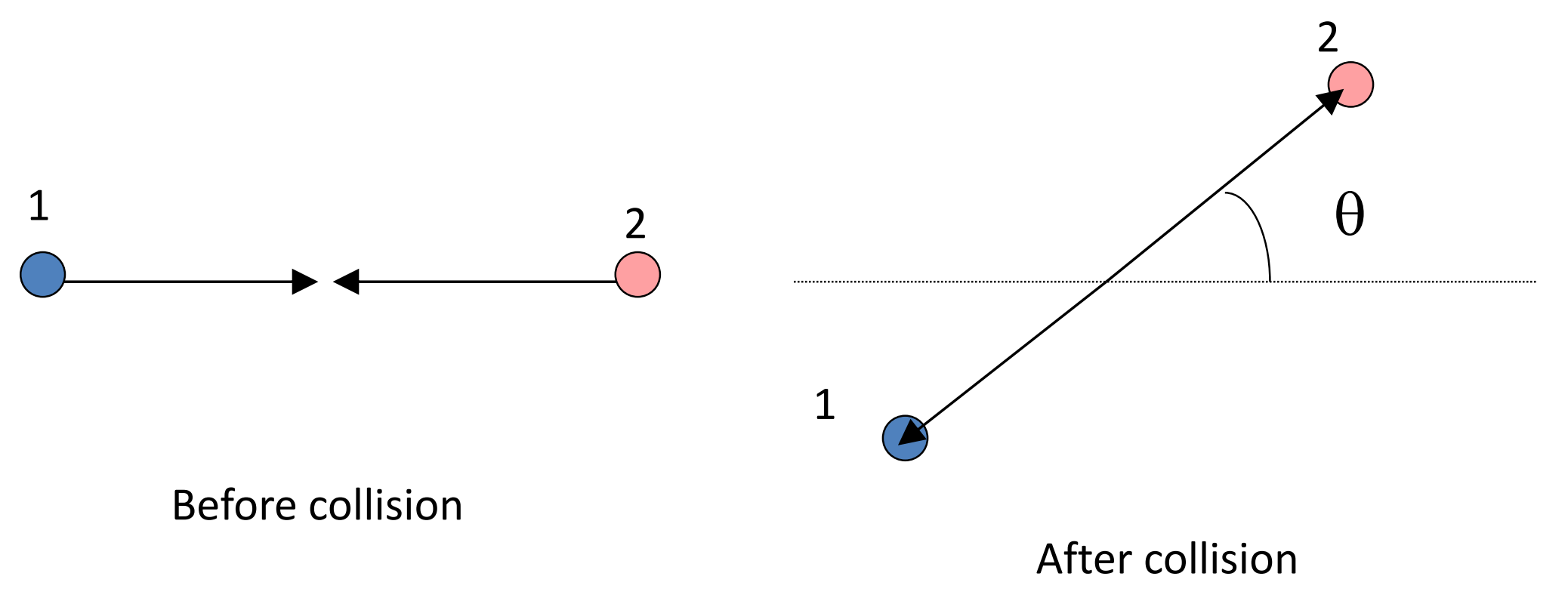

Center-of-mass system 


\section{General scattering theory}

\section{Different models}

Schrödinger equation: $H \Psi\left(\boldsymbol{r}_{1}, \boldsymbol{r}_{2}, \ldots \boldsymbol{r}_{\boldsymbol{A}}\right)=E \Psi\left(\boldsymbol{r}_{1}, \boldsymbol{r}_{2}, \ldots \boldsymbol{r}_{A}\right)$ with $E>0$ : scattering states

- A-body equation (microscopic models) $\quad H=\sum_{i} t_{i}+\frac{1}{2} \sum_{i, j} v_{i j}\left(\boldsymbol{r}_{\boldsymbol{i}}-\boldsymbol{r}_{\boldsymbol{j}}\right)$

$v_{i j}=$ nucleon-nucleon interaction

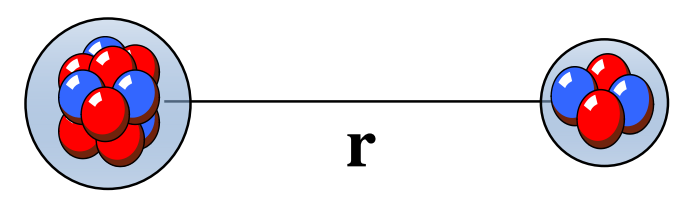

- Optical model: internal structure of the nuclei is neglected the particles interact by a nucleus-nucleus potential absorption simulated by the imaginary part = optical potential

$$
H \Psi(\boldsymbol{r})=\left(-\frac{\hbar^{2}}{2 \mu} \Delta+V(\boldsymbol{r})\right) \Psi(\boldsymbol{r})=E \Psi(\boldsymbol{r})
$$

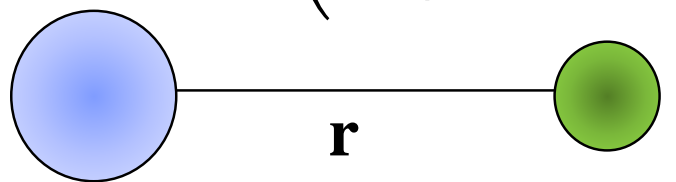

- Additional assumptions: elastic scattering no Coulomb interaction spins zero 


\section{General scattering theory}

\section{Potential/Optical model}

Two contributions to the nucleus-nucleus potential: nuclear $V_{N}(r)$ and Coulomb $V_{C}(r)$

Typical nuclear potential: $V_{N}(r)$ (short range, attractive)

- examples: Gaussian

Woods-Saxon:

$$
\begin{aligned}
& V_{N}(r)=-V_{0} \exp \left(-\left(r / r_{0}\right)^{2}\right) \\
& V_{N}(r)=-\frac{V_{0}}{1+\exp \left(\frac{r-r_{0}}{a}\right)}
\end{aligned}
$$

- Real at low energies

- parameters are fitted to experiment

- no analytical solution of the Schrödinger equation

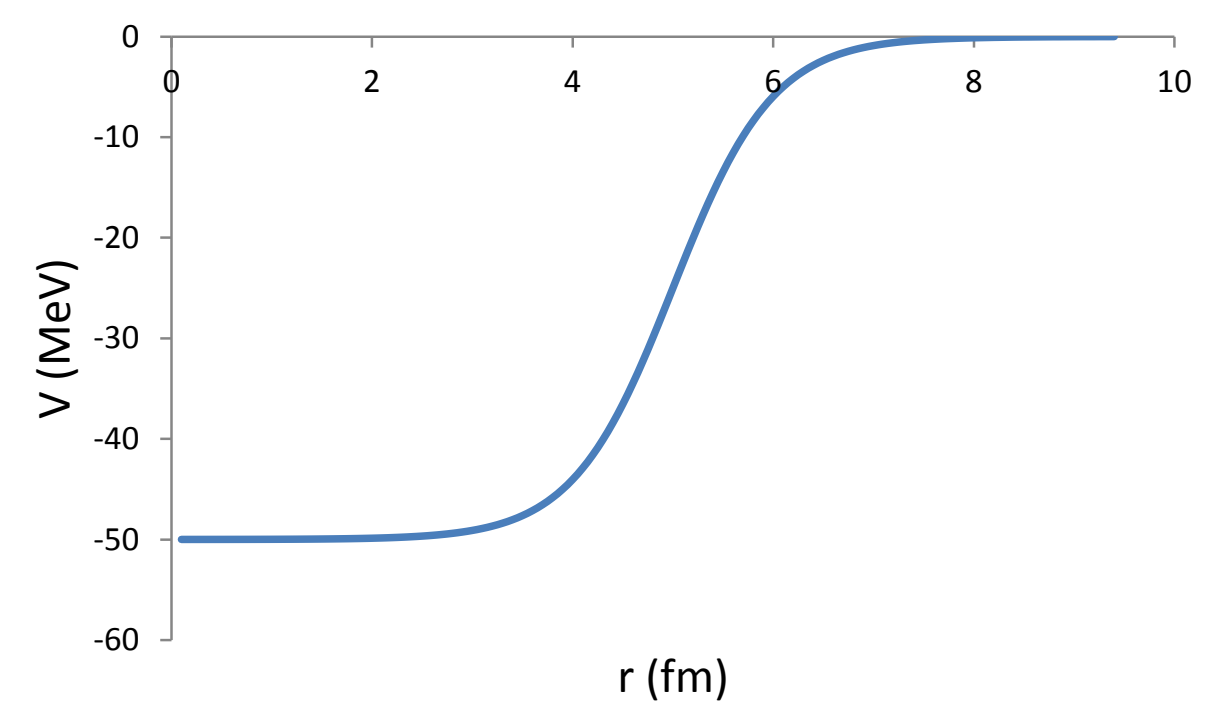

Woods-Saxon potential

$r_{0}=$ range ( $\sim$ sum of the radii)

$a=$ diffuseness $(\sim 0.5 \mathrm{fm})$

Figure: $V_{0}=50 \mathrm{MeV}, r_{0}=5 \mathrm{fm}, a=0.5 \mathrm{fm}$ 


\section{General scattering theory}

Coulomb potential: long range, repulsive

- «point-point » potential $: V_{C}(r)=\frac{Z_{1} z_{2} e^{2}}{r}$

- " point-sphere " potential : (radius $R_{C}$ )

$$
\begin{aligned}
& V_{C}(r)=\frac{Z_{1} Z_{2} e^{2}}{r} \text { for } r \geq R_{C} \\
& V_{C}(r)=\frac{Z_{1} Z_{2} e^{2}}{2 R_{C}}\left(3-\left(\frac{r}{R_{C}}\right)^{2}\right) \text { for } r \leq R_{C}
\end{aligned}
$$

Total potential : $V(r)=V_{N}(r)+V_{C}(r)$ : presents a maxium at the Coulomb barrier - radius $r=R_{B}$

- height $V\left(R_{B}\right)=E_{B}$

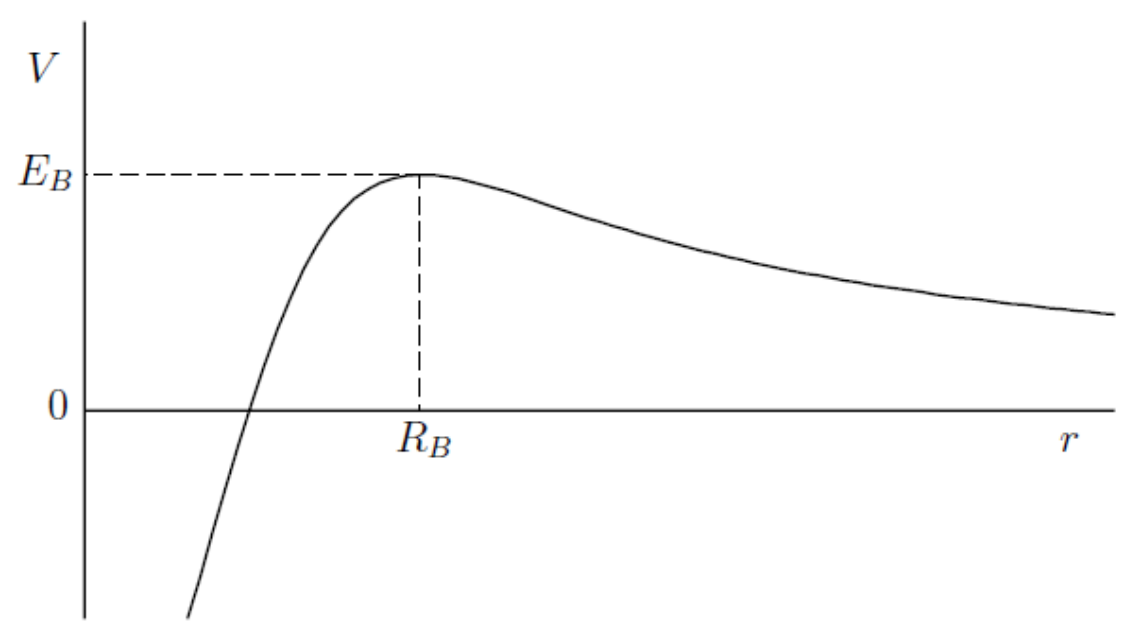




\section{General scattering theory}

\section{Scattering amplitude and cross section}

$$
H \Psi(\boldsymbol{r})=\left(-\frac{\hbar^{2}}{2 \mu} \Delta+V(\boldsymbol{r})\right) \Psi(\boldsymbol{r})=E \Psi(\boldsymbol{r})
$$

At large distances $: \Psi(\boldsymbol{r}) \rightarrow A\left(e^{i \boldsymbol{k} \cdot \boldsymbol{r}}+f(\theta) \frac{e^{i k r}}{r}\right) \quad$ (with $z$ along the beam axis)

where: $\quad k=$ wave number: $k^{2}=2 \mu E / \hbar^{2}$

$A$ =amplitude (scattering wave function is not normalized to unity)

$f(\theta)=$ scattering amplitude (length)

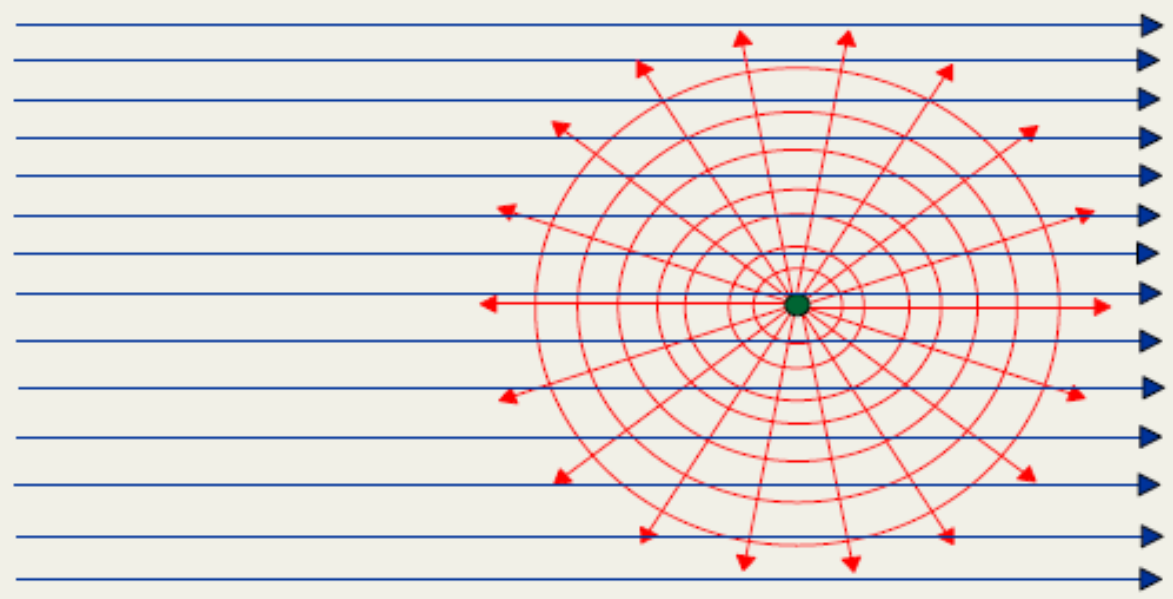

$\Phi(\mathrm{r} \rightarrow \infty)=A \mathrm{e}^{\mathrm{i} k \mathrm{z}}$

Incoming plane wave

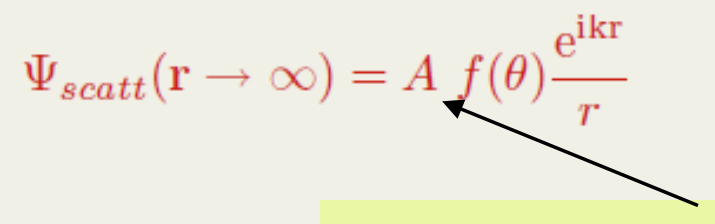




\section{General scattering theory}

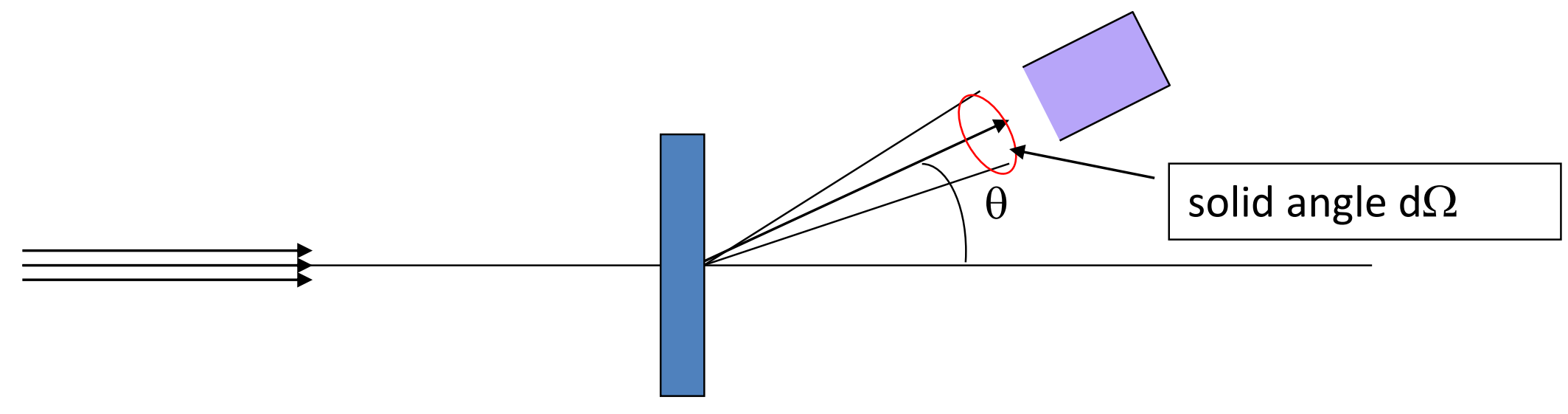

Cross section: $\quad \frac{d \sigma}{d \Omega}=|f(\theta)|^{2}, \sigma=\int \frac{d \sigma}{d \Omega} d \Omega$

- Cross section obtained from the asymptotic part of the wave function General problem for scattering states: the wave function must be known up to large distances

- "Direct" problem: determine $\sigma$ from the potential

- "Inverse" problem : determine the potential V from $\sigma$

- Angular distribution: $E$ fixed, $\theta$ variable

- Excitation function: $\theta$ variable, E fixed, 


\section{General scattering theory}

Main issue: determining the scattering amplitude $f(\theta)$ (and wave function $\Psi(\boldsymbol{r})$ )

At low energies: partial wave expansion: $\Psi(\boldsymbol{r})=\sum_{l m} \Psi_{l}(r) Y_{l}^{m}(\theta, \phi)$

- Scattering wave function necessary to compute cross sections

- Must be determined for each partial wave $l$

- Main interest: few partial waves at low energies

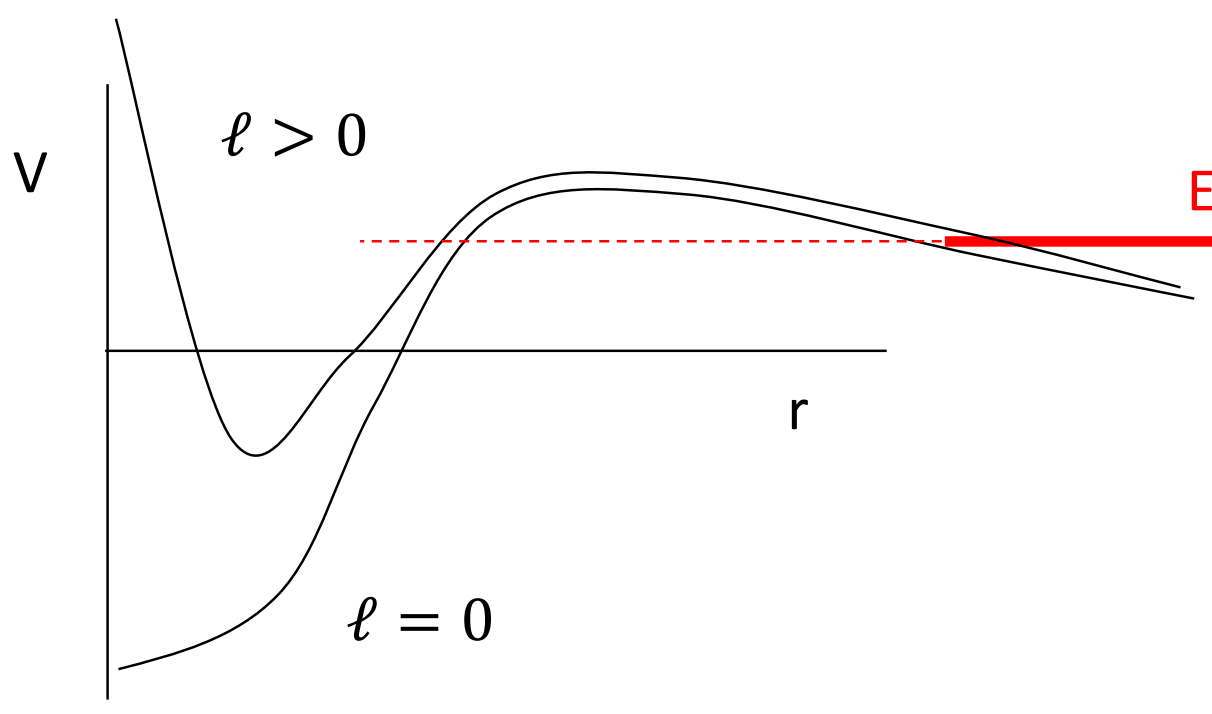

centrifugal term $: \frac{\hbar^{2}}{2 \mu} \frac{\ell(\ell+1)}{r^{2}}$

Partial-wave expansion

- Only a few partial waves contribute

- Effect more important for nucleonnucleus: $\mu \approx 1$

- Strongest for neutron: no barrier for $\ell=0$. 


\section{General scattering theory}

\section{Phase-shift method}

- Goal: solving the Schrodinger equation

$$
\left(-\frac{\hbar^{2}}{2 \mu} \Delta+V(\boldsymbol{r})\right) \Psi(\boldsymbol{r})=E \Psi(\boldsymbol{r})
$$

with a partial-wave expansion

$$
\Psi(\boldsymbol{r})=\sum_{\ell, m} \frac{u_{\ell}(r)}{r} Y_{\ell}^{m}\left(\Omega_{r}\right) Y_{\ell}^{m *}\left(\Omega_{k}\right)
$$

- Simplifying assumtions

- neutral systems (no Coulomb interaction)

- spins zero

- single-channel calculations $\rightarrow$ elastic scattering 


\section{General scattering theory}

- The wave function is expanded as

$$
\Psi(\boldsymbol{r})=\sum_{\ell, m} \frac{u_{\ell}(r)}{r} Y_{\ell}^{m}\left(\Omega_{r}\right) Y_{\ell}^{m *}\left(\Omega_{k}\right)
$$

- This provides the Schrödinger equation for each partial wave $\left(\Omega_{k}=0 \rightarrow m=0\right)$

$$
-\frac{\hbar^{2}}{2 \mu}\left(\frac{d^{2}}{d r^{2}}-\frac{\ell(\ell+1)}{r^{2}}\right) u_{\ell}+V(r) u_{\ell}=E u_{\ell}
$$

- Large distances : $r \rightarrow \infty, V(r) \rightarrow 0$

$u_{\ell}^{\prime \prime}-\frac{\ell(\ell+1)}{r^{2}} u_{\ell}+k^{2} u_{\ell}=0$ Bessel equation $\rightarrow u_{\ell}(r)=r j_{\ell}(k r), r n_{\ell}(k r)$

- Remarks

- must be solved for all $\ell$ values

- at low energies: few partial waves in the expansion

- at small $r: u_{\ell}(r) \rightarrow r^{\ell+1}$ 


\section{General scattering theory}

$$
\begin{aligned}
& \text { For small } \mathrm{x}: \quad j_{l}(x) \rightarrow \frac{x^{l}}{(2 l+1) ! !} \\
& n_{l}(x) \rightarrow-\frac{(2 l-1) ! !}{x^{l+1}} \\
& \text { For large } \mathrm{x}: \quad j_{l}(x) \rightarrow \frac{1}{x} \sin (x-l \pi / 2) \\
& n_{l}(x) \rightarrow-\frac{1}{x} \cos (x-l \pi / 2)
\end{aligned}
$$

Examples: $j_{0}(x)=\frac{\sin x}{x}, n_{0}(x)=-\frac{\cos x}{x}$

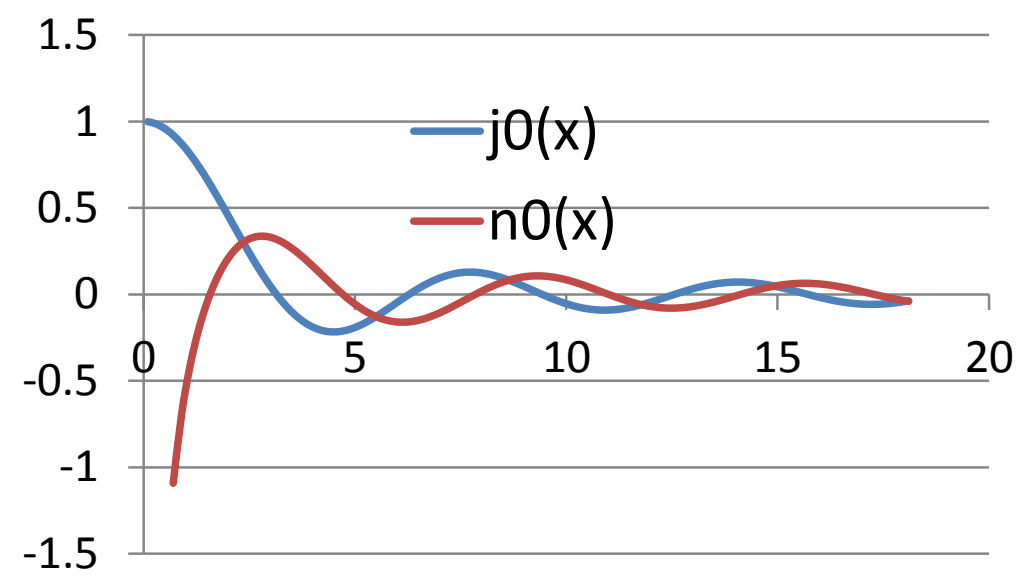

At large distances: $u_{\ell}(r)$ is a linear combination of $r j_{\ell}(k r)$ and $r n_{\ell}(k r)$

$$
u_{\ell}(r) \rightarrow C_{l} r\left(j_{\ell}(k r)-\tan \delta_{\ell} \times n_{\ell}(k r)\right)
$$

With $\delta_{\ell}=$ phase shift (provides information about the potential): If $\mathrm{V}=0 \rightarrow \delta_{\ell}=0$ 


\section{General scattering theory}

\section{Derivation of the elastic cross section}

- Identify the asymptotic behaviours

$$
\begin{aligned}
& \Psi(\boldsymbol{r}) \rightarrow A\left(e^{i \boldsymbol{k} \cdot \boldsymbol{r}}+f(\theta) \frac{e^{i k r}}{r}\right) \\
& \Psi(\boldsymbol{r}) \rightarrow \sum_{\ell} C_{\ell}\left(j_{\ell}(k r)-\tan \delta_{\ell} \times n_{\ell}(k r)\right) Y_{\ell}^{0}\left(\Omega_{r}\right) \sqrt{\frac{2 \ell+1}{4 \pi}}
\end{aligned}
$$

- Provides coefficients $C_{\ell}$ and scattering amplitude $f(\theta)$ (elastic scattering)

$$
\begin{aligned}
& f(\theta, E)=\frac{1}{2 i k} \sum_{\ell=0}^{\infty}(2 \ell+1)\left(\exp \left(2 i \delta_{\ell}(E)\right)-1\right) P_{\ell}(\cos \theta) \\
& \frac{d \sigma(\theta, E)}{d \Omega}=|f(\theta, E)|^{2}
\end{aligned}
$$

- Integrated cross section (neutral systems only)

$$
\sigma=\frac{\pi}{k^{2}} \sum_{\ell=0}^{\infty}(2 \ell+1) \sin ^{2} \delta_{\ell}
$$

- In practice, the summation over $\ell$ is limited to some $\ell_{\max }$ 


\section{General scattering theory}

$$
\frac{d \sigma(\theta, E)}{d \Omega}=|f(\theta, E)|^{2} \text { with } f(\theta, E)=\frac{1}{2 i k} \sum_{\ell=0}^{\infty}(2 \ell+1)\left(\exp \left(2 i \delta_{\ell}(E)\right)-1\right) P_{\ell}(\cos \theta)
$$

$\rightarrow$ factorization of the dependences in $E$ and $\theta$ low energies: small number of $\ell$ values ( $\delta_{\ell} \rightarrow 0$ when $\ell$ increases) high energies: large number ( $\rightarrow$ alternative methods)

\section{General properties of the phase shifts}

1. The phase shift (and all derivatives) are continuous functions of $E$

2. The phase shift is known within $n \pi: \exp 2 i \delta=\exp (2 i(\delta+n \pi))$

3. Levinson theorem

- $\delta_{\ell}(E=0)$ is arbitrary

- $\delta_{\ell}(0)-\delta_{\ell}(\infty)=\mathrm{N} \pi$, where $\mathrm{N}$ is the number of bound states in partial wave $\ell$

- Example: $p+n$,

$$
\begin{aligned}
& \ell=0: \delta_{0}(0)-\delta_{0}(\infty)=\pi(\text { bound deuteron) } \\
& \left.\ell=1: \delta_{1}(0)-\delta_{1}(\infty)=0 \text { (no bound state for } \ell=1\right)
\end{aligned}
$$




\section{General scattering theory}

- Example: hard sphere (radius a)

- continuity at $r=a \rightarrow j_{\ell}(k a)-\tan \delta_{\ell} \times n_{\ell}(k a)=0 \quad \rightarrow \tan \delta_{\ell}=\frac{j_{\ell}(k a)}{n_{\ell}(k a)}$

$$
\rightarrow \delta_{0}=-k a
$$
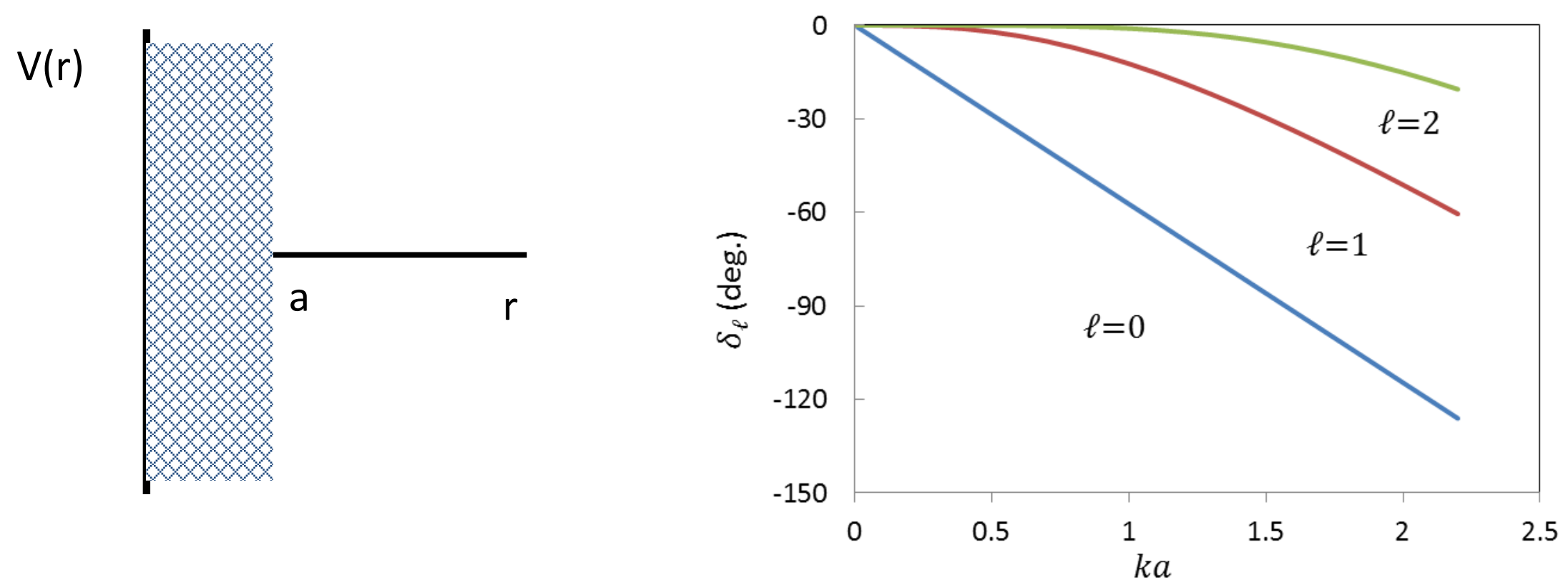

At low energies: $\quad \delta_{\ell}(E) \rightarrow-\frac{(k a)^{2 \ell+1}}{(2 \ell+1) !(2 l-1) ! !}$, in general: $\delta_{\ell}(E) \sim k^{2 \ell+1}$

$\rightarrow$ Strong difference between $\ell=0$ (no barrier) et $\ell \neq 0$ (centrifugal barrier)

(typical to neutron-induced reactions) 


\section{General scattering theory}

example : $\alpha+\mathrm{n}$ phase shift $\ell=0$

consistent with the hard sphere $(a \sim 2.2 \mathrm{fm})$

R. A. ARNDT AND L, D, ROPER

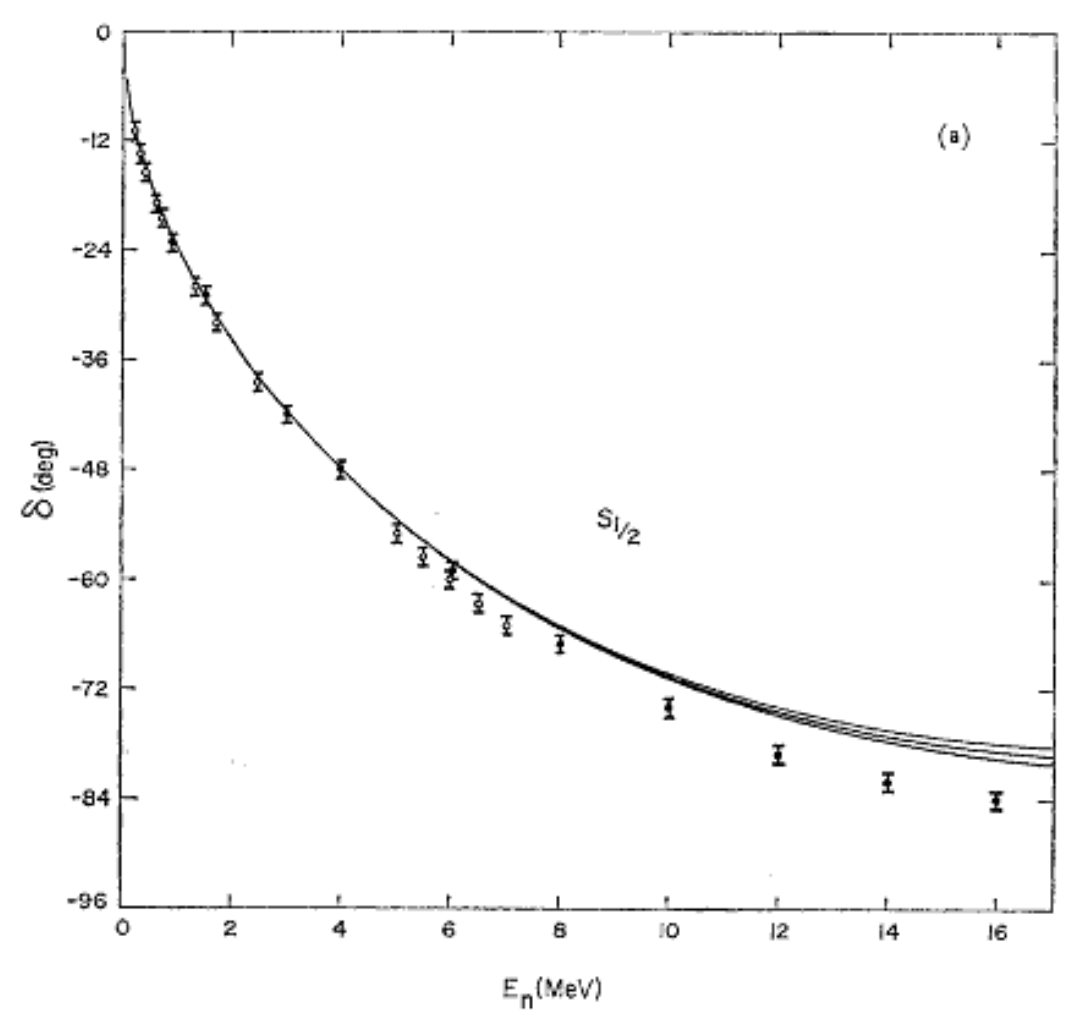




\section{General scattering theory}

\section{Resonances}

Resonances: $\delta_{R}(E) \approx \operatorname{atan} \frac{\Gamma}{2\left(E_{R}-E\right)}=$ Breit-Wigner approximation

$\mathrm{E}_{\mathrm{R}}=$ resonance energy

$\Gamma=$ resonance width: related to the lifetime $\Gamma \tau=\hbar$

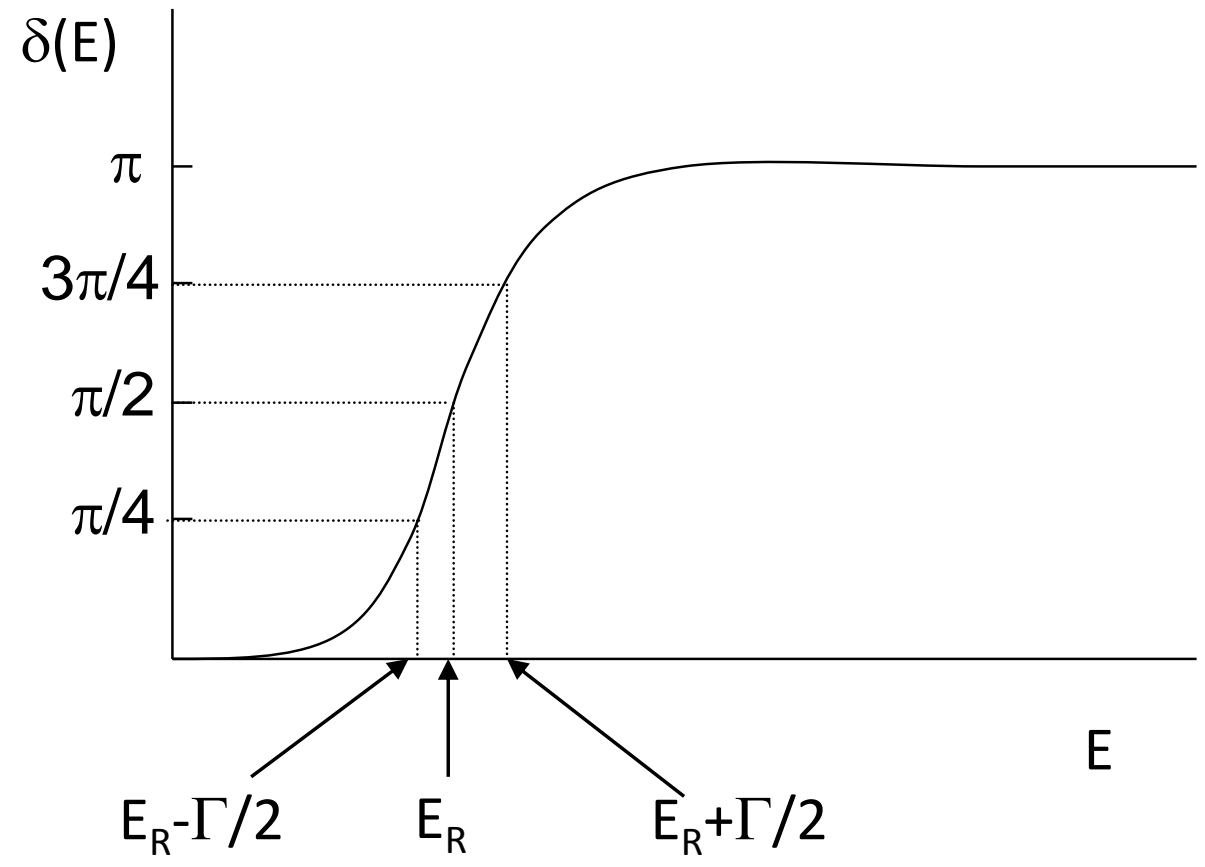

- Narrow resonance: $\Gamma$ small, $\tau$ large

- Broad resonance: $\Gamma$ large, $\tau$ small

- Bound states: $\Gamma=0, E_{R}<0$ 


\section{General scattering theory}

Cross section (for neutrons)

$\sigma(E)=\frac{\pi}{k^{2}} \sum_{\ell}(2 \ell+1)\left|\exp \left(2 i \delta_{\ell}\right)-1\right|^{2}$ maximum for $\delta=\frac{\pi}{2}$

Near the resonance: $\sigma(E) \approx \frac{4 \pi}{k^{2}}\left(2 \ell_{R}+1\right) \frac{\Gamma^{2} / 4}{\left(E_{R}-E\right)^{2}+\Gamma^{2} / 4}$, where $\ell_{R}=$ resonant partial wave

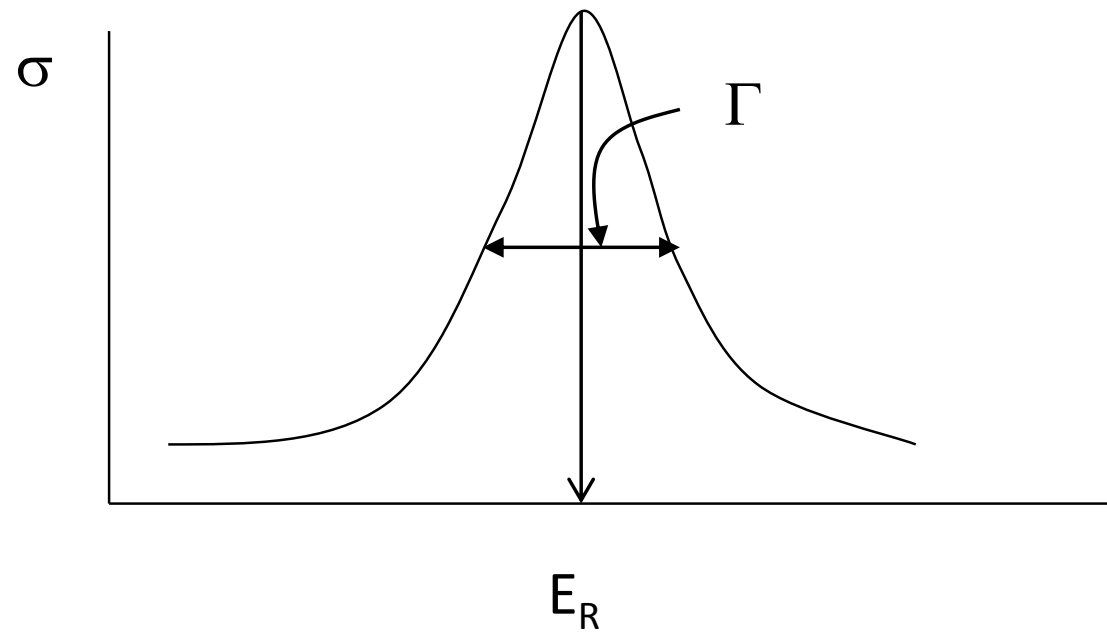

In practice:

- Peak not symmetric ( $\Gamma$ depends on $E$ )

- «Background » neglected (other $\ell$ values)

- Differences with respect to Breit-Wigner 


\section{General scattering theory}

Example: $n+{ }^{12} \mathrm{C}$

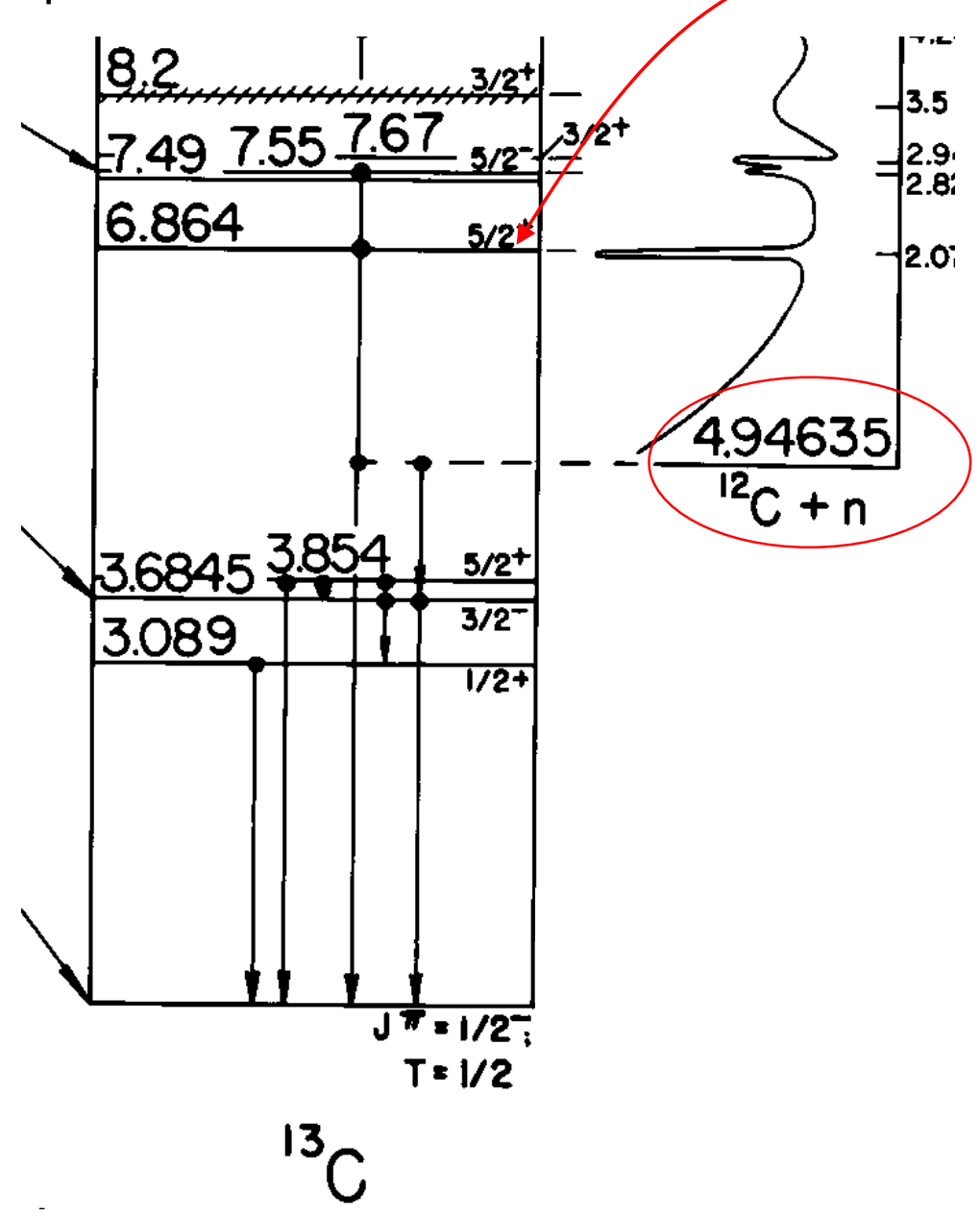

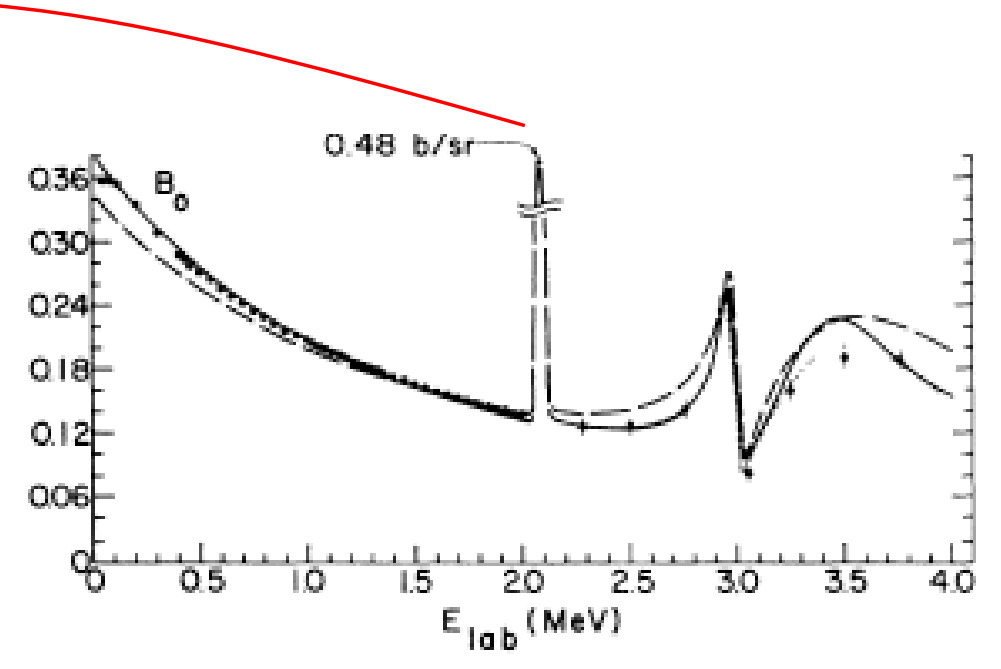

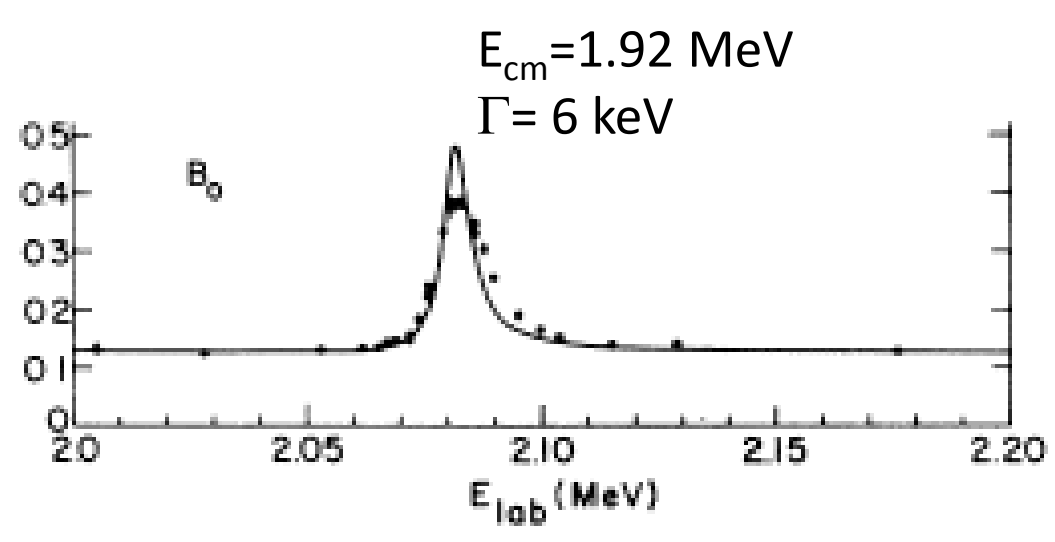

Comparison of 2 typical times:

a. Lifetime of the resonance: $\tau_{R}=\hbar / \Gamma \approx \frac{197}{3.10^{23} \times 6.10^{-3}} \approx 1.1 \times 10^{-19} \mathrm{~S}$

b. Interaction time without resonance: $\tau_{N R}=d / v \approx 5.2 \times 10^{-22} s \rightarrow \tau_{\mathrm{NR}}<<\tau_{\mathrm{R}}$ 


\section{General scattering theory}

\section{Narrow resonances}

- Small particle width

- long lifetime

- can be approximetly treated by neglecting the asymptotic behaviour of the wave function

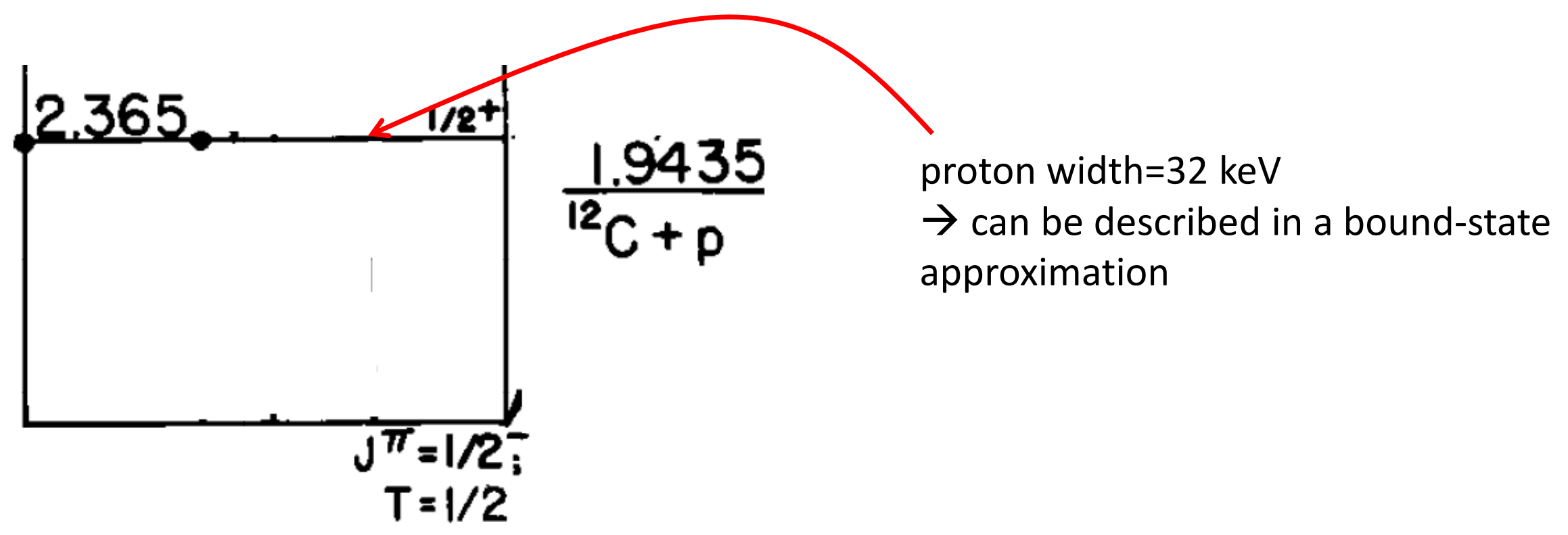




\section{General scattering theory}

\section{Broad resonances}

- Large particle width

- Short lifetime

- asymptotic behaviour of the wave function is important

$\rightarrow$ rigorous scattering theory

$\rightarrow$ bound-state model complemented by other tools (complex scaling, etc.)

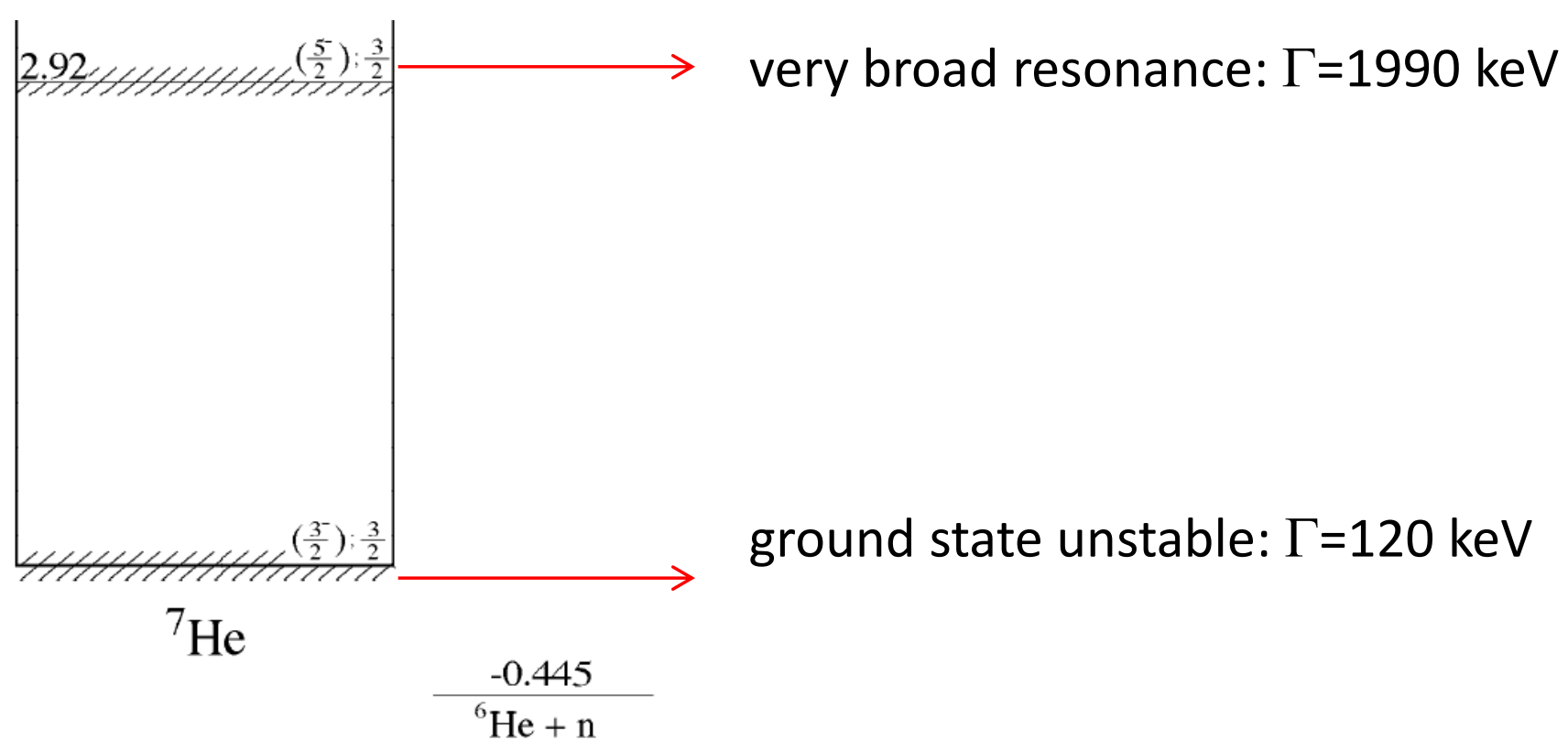




\section{Generalizations}

- Extension to charged systems

- Numerical calculation

- Optical model (high energies $\rightarrow$ absorption)

- Extension to multichannel problems 


\section{Generalizations}

\section{Generalization 1: charged systems}

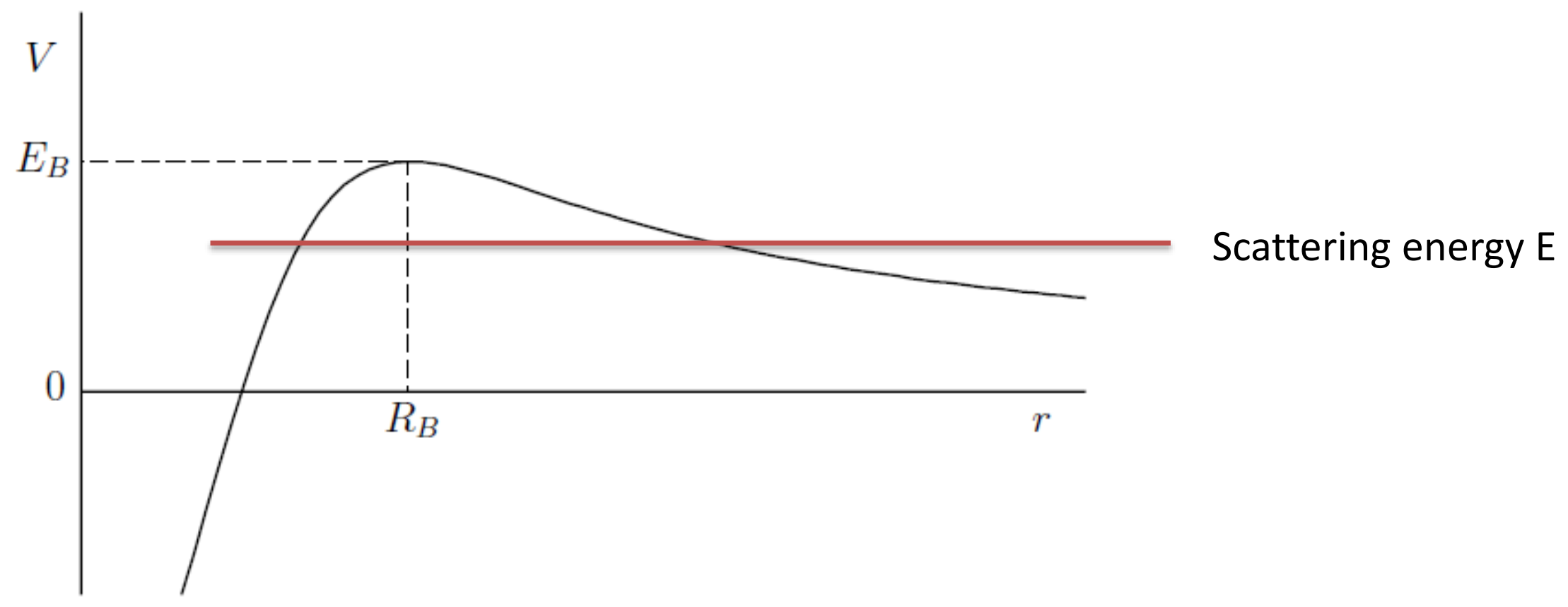

$E \gg E_{B}$ : weak coulomb effects ( $V$ negligible with respect to $E$ ) $E<E_{B}$ : strong coulomb effects (ex: nuclear astrophysics) 


\section{Generalizations}

\section{A. Asymptotic behaviour}

\begin{tabular}{|c|} 
Neutral systems \\
$\left(-\frac{\hbar^{2}}{2 \mu} \Delta+V_{N}(r)-E\right) \Psi(\boldsymbol{r})=0$ \\
$\Psi(\boldsymbol{r}) \rightarrow \exp (i \boldsymbol{k} \cdot \boldsymbol{r})+f(\theta) \frac{\exp (i k r)}{r}$
\end{tabular}

\begin{tabular}{|l|} 
Charged systems \\
$\left(-\frac{\hbar^{2}}{2 \mu} \Delta+V_{N}(r)+\frac{Z_{1} Z_{2} e^{2}}{r}-E\right) \Psi(\boldsymbol{r})=0$ \\
$\Psi(\boldsymbol{r})$ \\
$\rightarrow \exp (i \boldsymbol{k} \cdot \boldsymbol{r}+i \eta \ln (\boldsymbol{k} \cdot \boldsymbol{r}-k r))$ \\
$+f(\theta) \frac{\exp (i(k r-\eta \ln 2 k r))}{r}$ \\
$\eta=\frac{Z_{1} Z_{2} e^{2}}{\hbar v}$ \\
- Sommerfeld parameter \\
- In measurement » of coulomb effects \\
- Decreases at low energies
\end{tabular}




\section{Generalizations}

B. Phase shifts with the coulomb potential

Neutral system: $\quad\left(\frac{d^{2}}{d r^{2}}-\frac{\ell(\ell+1)}{r^{2}}+k^{2}\right) R_{\ell}=0$

Bessel equation : solutions $j_{\ell}(k r), n_{\ell}(k r)$

Charged system: $\quad\left(\frac{d^{2}}{d r^{2}}-\frac{\ell(\ell+1)}{r^{2}}-2 \frac{\eta k}{r}+k^{2}\right) R_{\ell}=0$ :

Coulomb equation: solutions $F_{\ell}(\eta, k r), G_{\ell}(\eta, k r)$
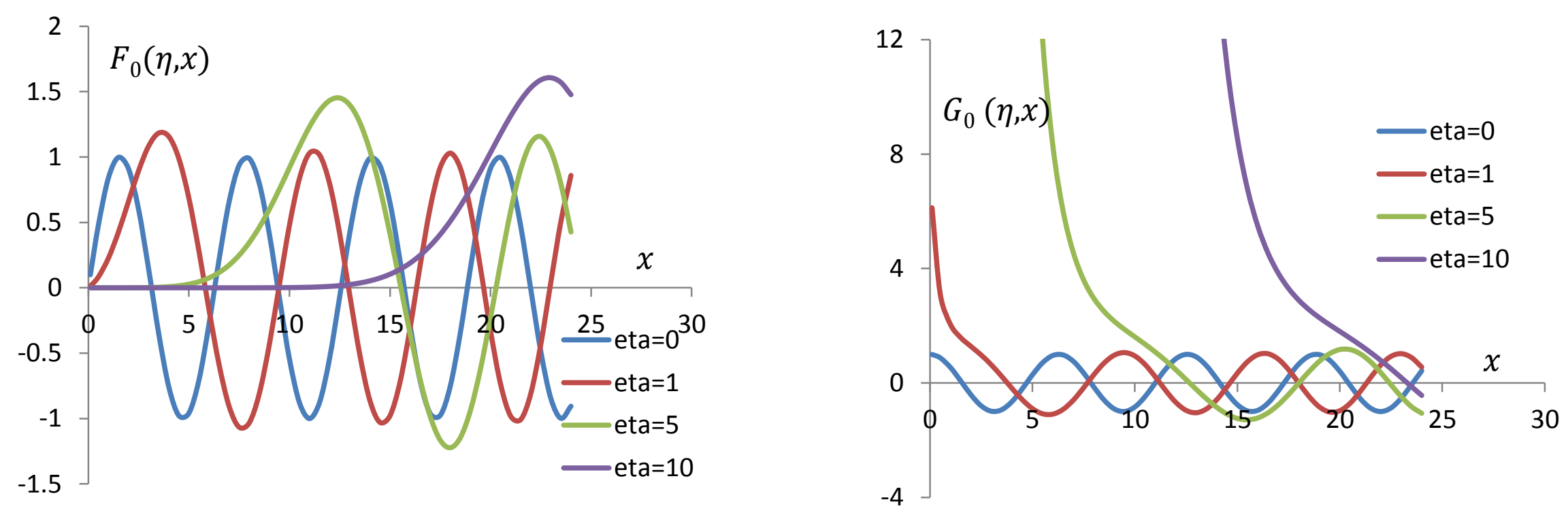


\section{Generalizations}

- Incoming and outgoing functions (complex)

$I_{\ell}(\eta, x)=G_{\ell}(\eta, x)-i F_{\ell}(\eta, x) \rightarrow e^{-i\left(x-\frac{\ell \pi}{2}-\eta \ln 2 x+\sigma_{\ell}\right)}$ : incoming wave

$o_{\ell}(\eta, x)=G_{\ell}(\eta, x)+i F_{\ell}(\eta, x) \rightarrow e^{i\left(x-\frac{\ell \pi}{2}-\eta \ln 2 x+\sigma_{\ell}\right)}$ : outgoing wave

- Phase-shift definition

$\circ$ neutral systems $: R_{\ell}(r) \rightarrow r A\left(j_{\ell}(k r)-\tan \delta_{\ell} n_{\ell}(k r)\right)$

○ charged systems: $R_{\ell}(r) \rightarrow A\left(F_{\ell}(\eta, k r)+\tan \delta_{\ell} G_{\ell}(\eta, k r)\right)$

$$
\begin{aligned}
& \rightarrow B\left(\cos \delta_{\ell} F_{\ell}(\eta, k r)+\sin \delta_{\ell} G_{\ell}(\eta, k r)\right. \\
& \rightarrow C\left(I_{\ell}(\eta, k r)-U_{\ell} O_{\ell}(\eta, k r)\right)
\end{aligned}
$$

3 equivalent definitions (amplitude is different)

Collision matrix (=scattering matrix)

$$
U_{\ell}=e^{2 i \delta_{\ell}}: \text { modulus }\left|U_{\ell}\right|=1
$$




\section{Generalizations}

Example: hard-sphere potential

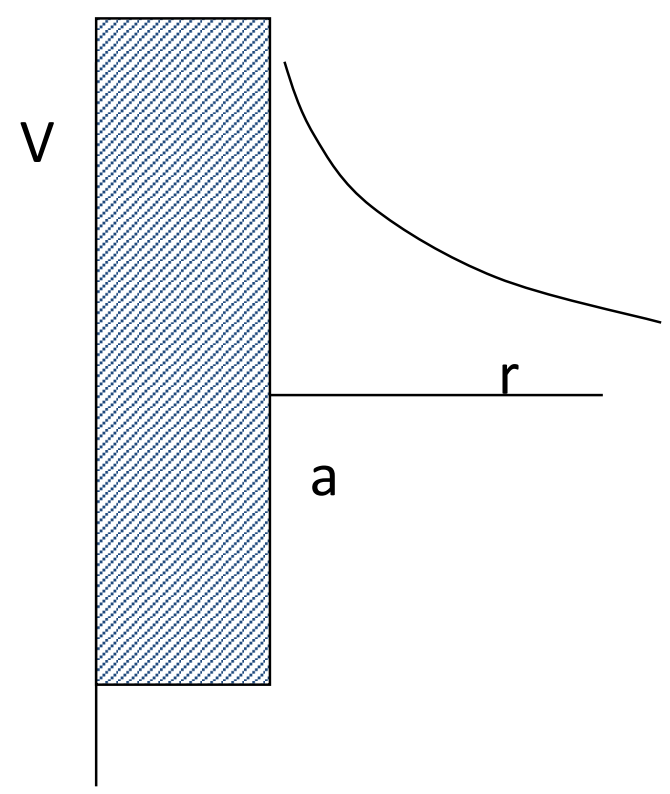

$$
\begin{aligned}
& V(r)=\frac{Z_{1} Z_{2} e^{2}}{r} \text { for } r>a \\
& \infty \text { for } r<a \\
& \text { phase shift: } \tan \delta_{\ell}=-\frac{F_{\ell}(\eta, k a)}{G_{\ell}(\eta, k a)}
\end{aligned}
$$

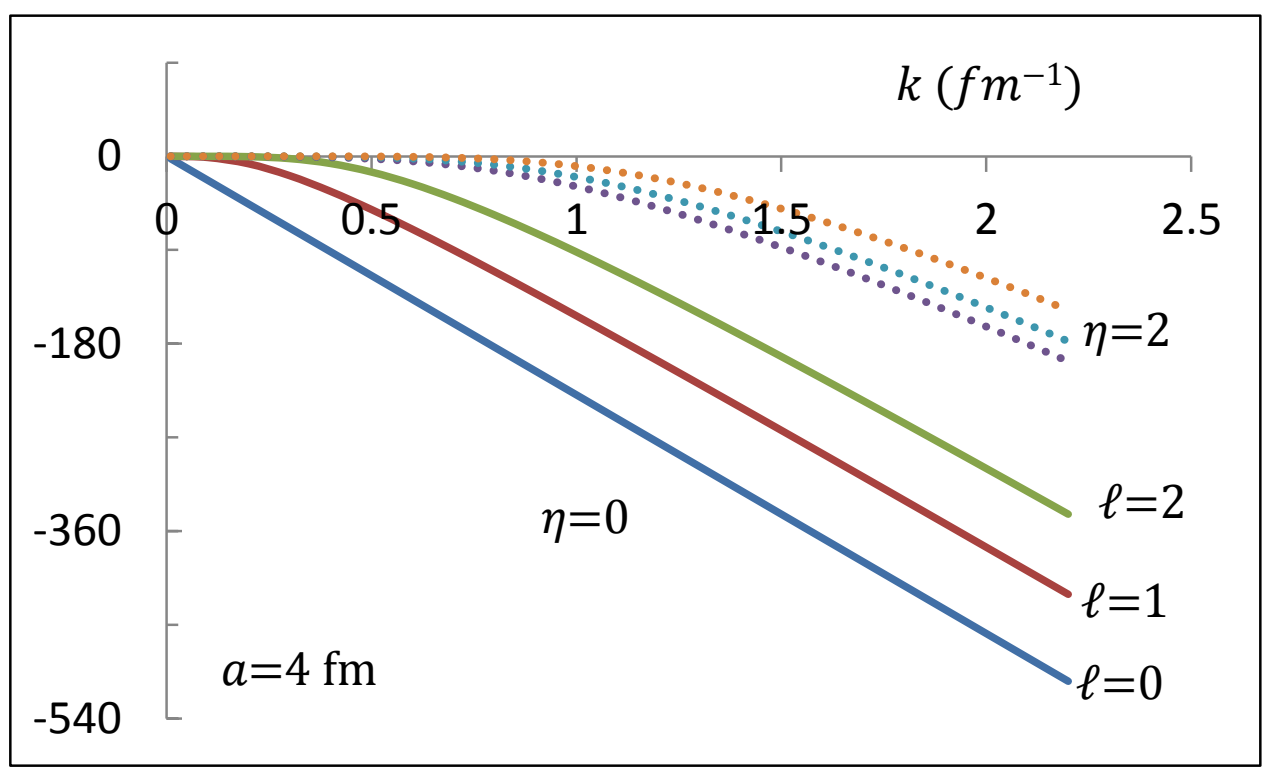




\section{Generalizations}

\section{Rutherford cross section}

For a Coulomb potential $\left(V_{N}=0\right)$ :

- $\quad$ scattering amplitude : $f_{c}(\theta)=-\frac{\eta}{2 k \sin ^{2} \theta / 2} e^{2 i\left(\sigma_{0}-\eta \ln \sin \theta / 2\right)}$

- Coulomb phase shift for $\ell=0: \sigma_{0}=\arg \Gamma(1+i \eta)$

We get the Rutherford cross section:

$$
\frac{d \sigma_{C}}{d \Omega}=\left|f_{c}(\theta)\right|^{2}=\left(\frac{Z_{1} Z_{2} e^{2}}{4 E \sin ^{2} \theta / 2}\right)^{2}
$$

- Increases at low energies

- Diverges at $\theta=0 \rightarrow$ no integrated cross section 


\section{Generalizations}

D. Cross sections with nuclear and Coulomb potentials

- The general defintions

are still valid

$$
\begin{aligned}
& f(\theta)=\frac{1}{2 i k} \sum_{\ell=0}^{\infty}(2 \ell+1)\left(\exp \left(2 i \delta_{\ell}\right)-1\right) P_{\ell}(\cos \theta) \\
& \frac{d \sigma}{d \Omega}=|f(\theta)|^{2}
\end{aligned}
$$

- Problem : very slow convergence with $\ell$

$\rightarrow$ separation of the nuclear and coulomb phase shifts

$$
\begin{aligned}
& \delta_{\ell}=\delta_{\ell}^{N}+\sigma_{\ell} \\
& \sigma_{\ell}=\arg \Gamma(1+\ell+i \eta)
\end{aligned}
$$

- Scattering amplitude $f(\theta)$ written as $f(\theta)=f^{C}(\theta)+f^{N}(\theta)$

- $f^{C}(\theta)=\frac{1}{2 i k} \sum_{\ell=0}^{\infty}(2 \ell+1)\left(\exp \left(2 i \sigma_{\ell}\right)-1\right) P_{\ell}(\cos \theta)=-\frac{\eta}{2 k \sin ^{2} \theta / 2} e^{2 i\left(\sigma_{0}-\eta \ln \sin \theta / 2\right)}$

$\rightarrow$ analytical

- $f^{N}(\theta)=\frac{1}{2 i k} \sum_{\ell=0}^{\infty}(2 \ell+1) \exp \left(2 i \sigma_{\ell}\right)\left(\exp \left(2 i \delta_{\ell}^{N}\right)-1\right) P_{\ell}(\cos \theta)$

$\rightarrow$ converges rapidly 


\section{Generalizations}

Total cross section: $\frac{d \sigma}{d \Omega}=|f(\theta)|^{2}=\left|f^{C}(\theta)+f^{N}(\theta)\right|^{2}$

- Nuclear term dominant at $180^{\circ}$

- Coulomb term coulombien dominant at small angles $\rightarrow$ used to normalize experiments

- Coulomb amplitude strongly depends on the angle $\rightarrow \frac{d \sigma / d \Omega}{d \sigma_{C} / d \Omega}$

- Integrated cross section $\int \frac{d \sigma}{d \Omega} \mathrm{d} \Omega$ is not defined

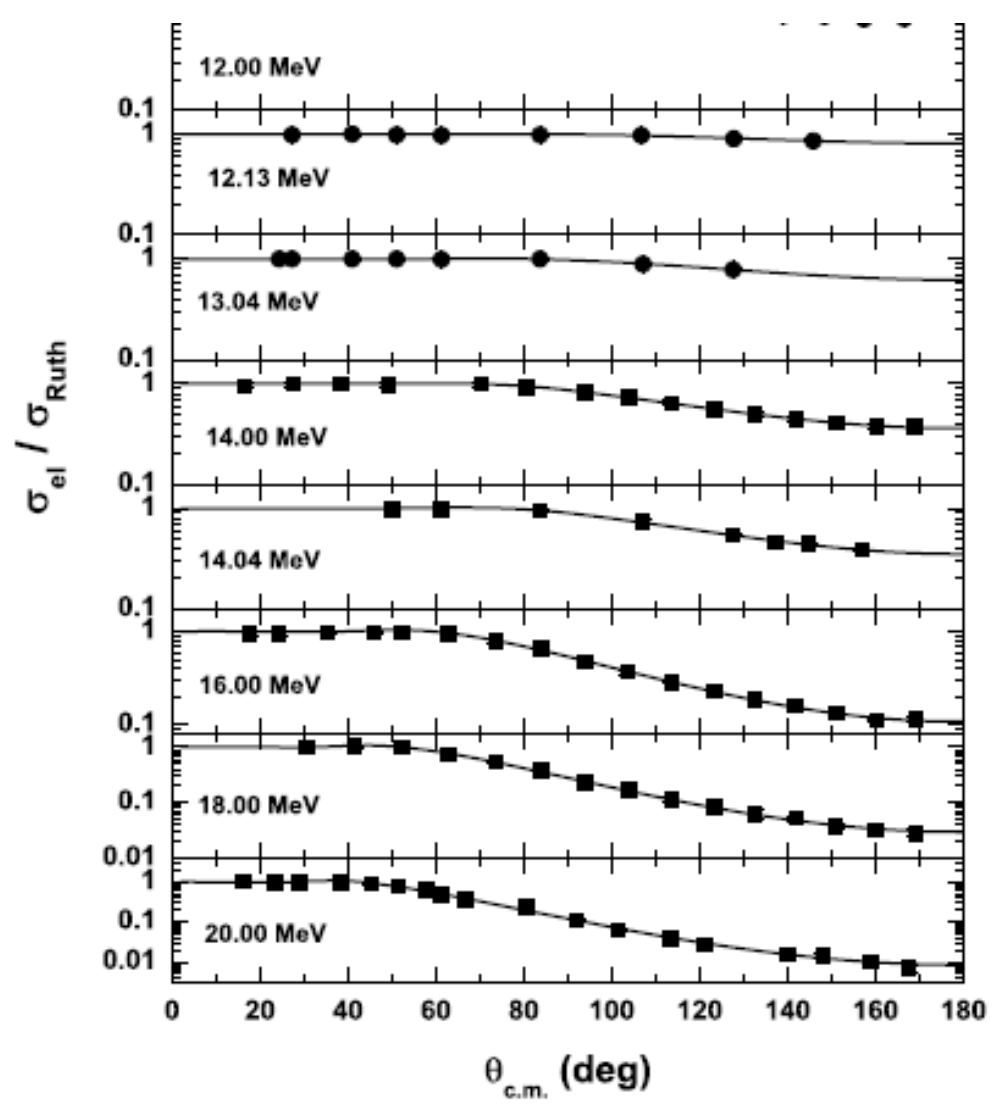

\section{System ${ }^{6} \mathrm{Li}+{ }^{58} \mathrm{Ni}$ \\ - $E_{c m}=\frac{58}{64} E_{l a b}$ \\ - Coulomb barrier}

$$
E_{B} \sim \frac{3 * 28 * 1.44}{7} \sim 17 \mathrm{MeV}
$$

- Below the barrier: $\sigma \sim \sigma_{C}$

- Above $E_{B}: \sigma$ is different from $\sigma_{C}$ 


\section{Generalizations}

\section{Generalization 2: numerical calculation}

For some potentials: analytic solution of the Schrödinger equation

In general: no analytical solution $\rightarrow$ numerical approach

$$
\begin{aligned}
& -\frac{\hbar^{2}}{2 \mu} \frac{d^{2}}{d r^{2}} u_{\ell}(r)+(V(r)-E) u_{\ell}(r)=0 \\
& \text { with: } \begin{aligned}
V(r) & =V_{N}(r)+\frac{Z_{1} Z_{2} e^{2}}{r}+\frac{\hbar^{2}}{2 \mu} \frac{\ell(\ell+1)}{r^{2}} \\
u_{\ell}(r) & \rightarrow F_{\ell}(k r, \eta) \cos \delta_{\ell}+G_{\ell}(k r, \eta) \sin \delta_{\ell}
\end{aligned}
\end{aligned}
$$

Numerical solution : discretization $\mathrm{N}$ points, with mesh size $\mathrm{h}$

- $u_{l}(0)=0$

- $u_{l}(h)=1$ (or any constant)

- $u_{l}(2 h)$ is determined numerically from $u_{l}(0)$ and $u_{l}(h)$ (Numerov algorithm)

- $u_{l}(3 h), \ldots u_{l}(N h)$

- for large $r$ : matching to the asymptotic behaviour $\rightarrow$ phase shift

Bound states: same idea (but energy is unknown) 


\section{Generalizations}

\section{ᄃ Example: $\alpha+\alpha$}

Experimental spectrum of ${ }^{8} \mathrm{Be}$

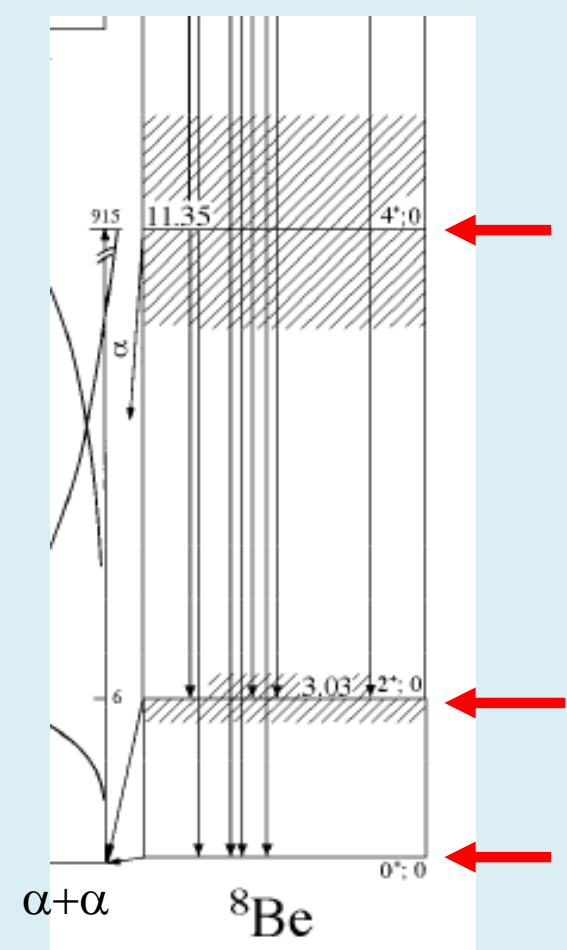

\section{Experimental phase shifts}

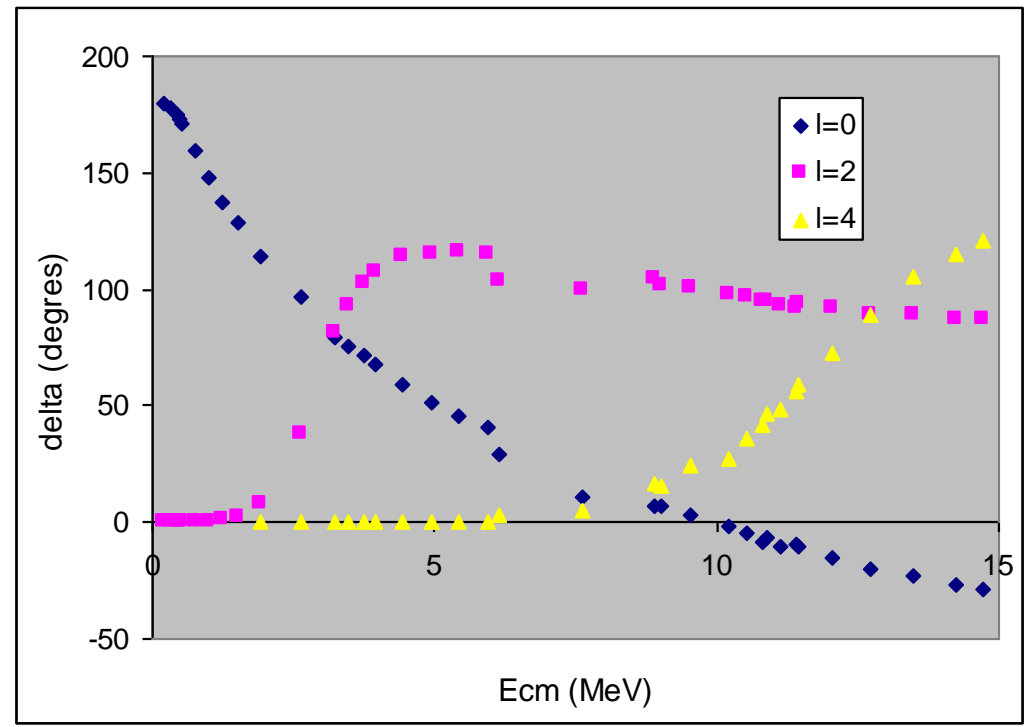

Potential: $V_{N}(r)=-122.3^{*} \exp \left(-(r / 2.13)^{2}\right)$

$2^{+}$

E 3 MeV

$\Gamma \sim 1.5 \mathrm{MeV}$

$0^{+}$

$\mathrm{E}=0.09 \mathrm{MeV}$

$\Gamma=6 \mathrm{eV}$

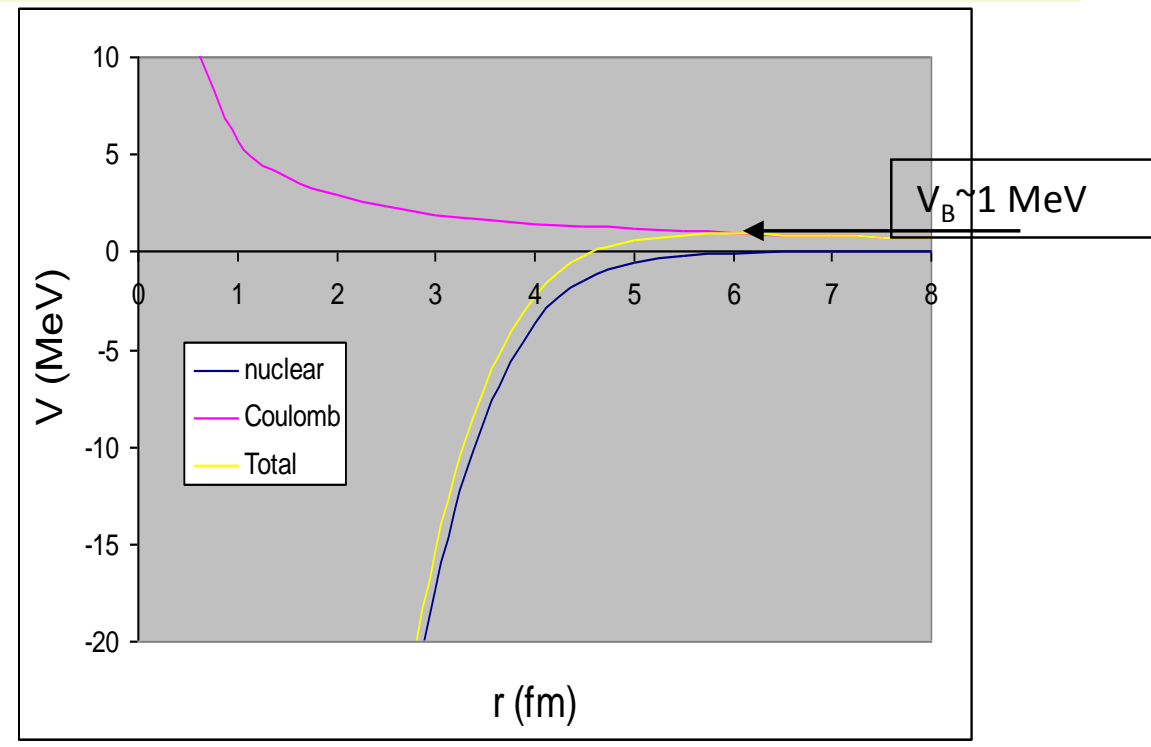




\section{Generalizations}

$\alpha+\alpha$ wave function for $\ell=0$

$\alpha-\alpha$ potential

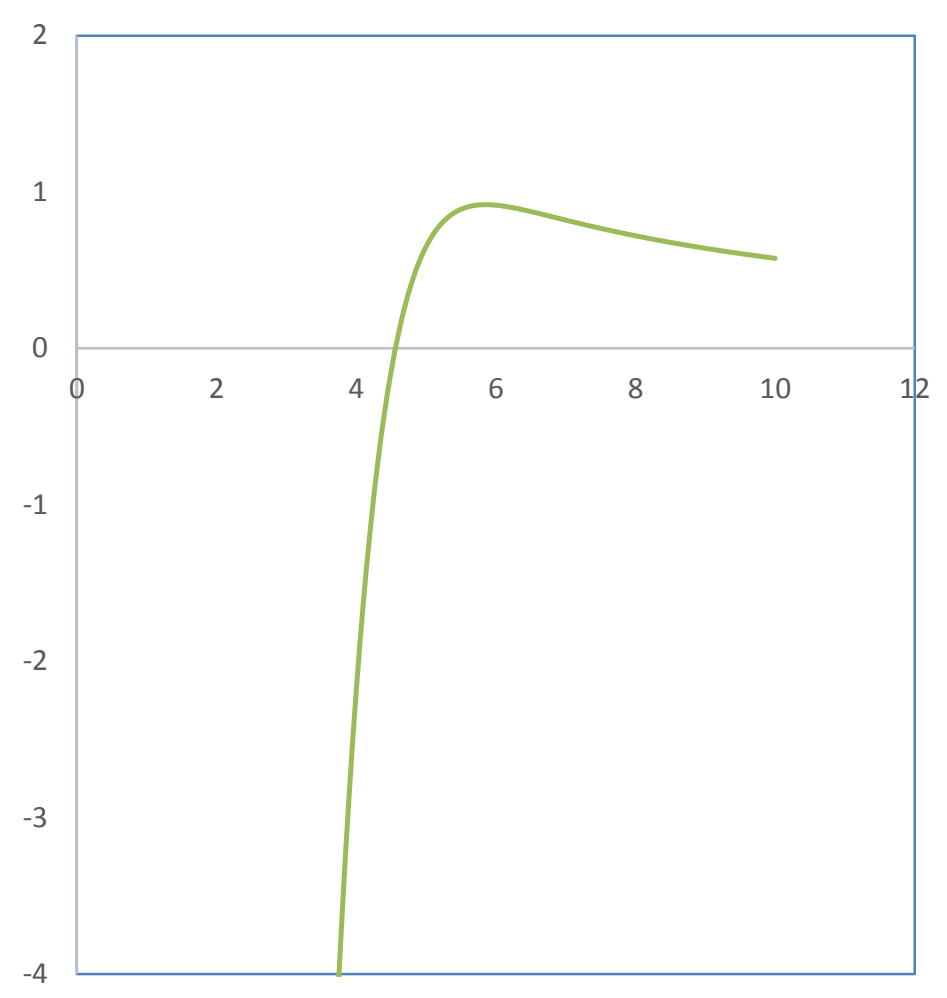

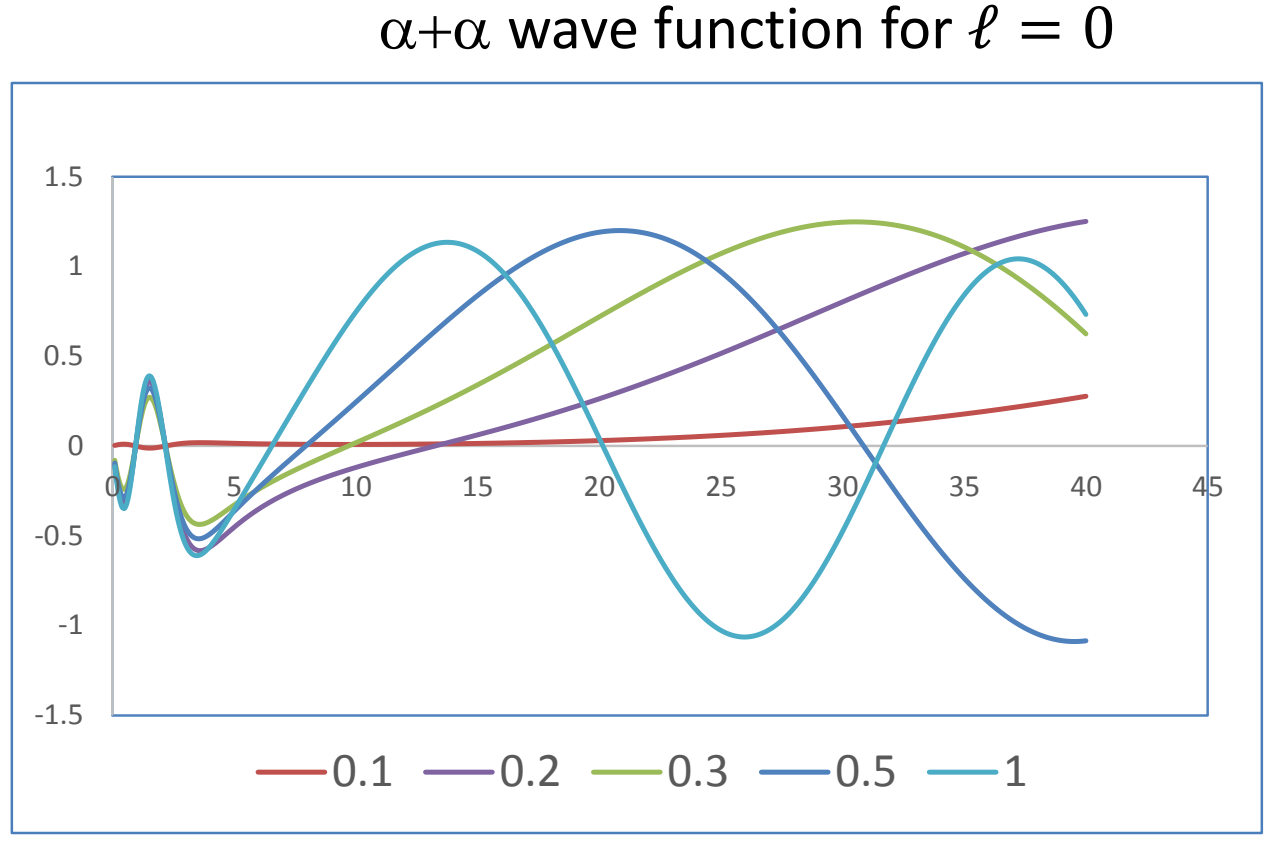

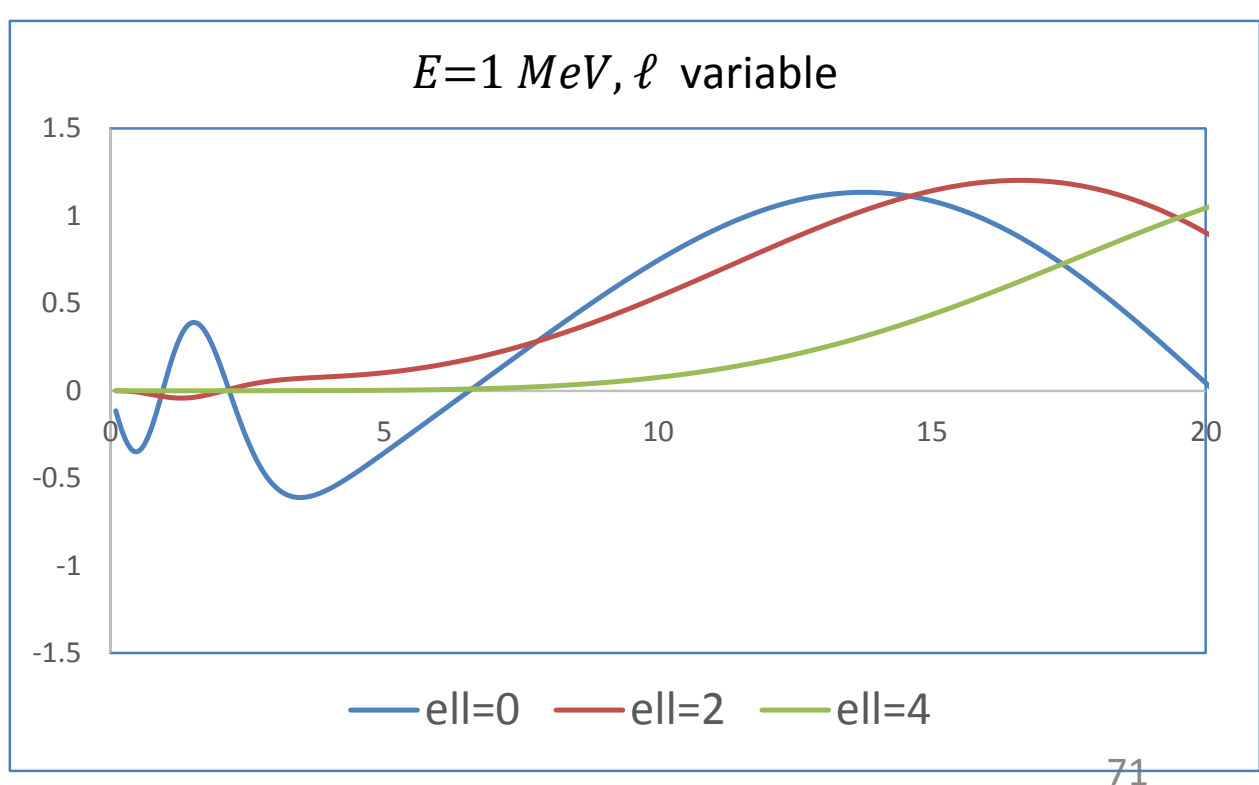




\section{Generalizations}

Generalization 3: complex potentials $V=V_{R}+i W$

Goal: to simulate absorption channels

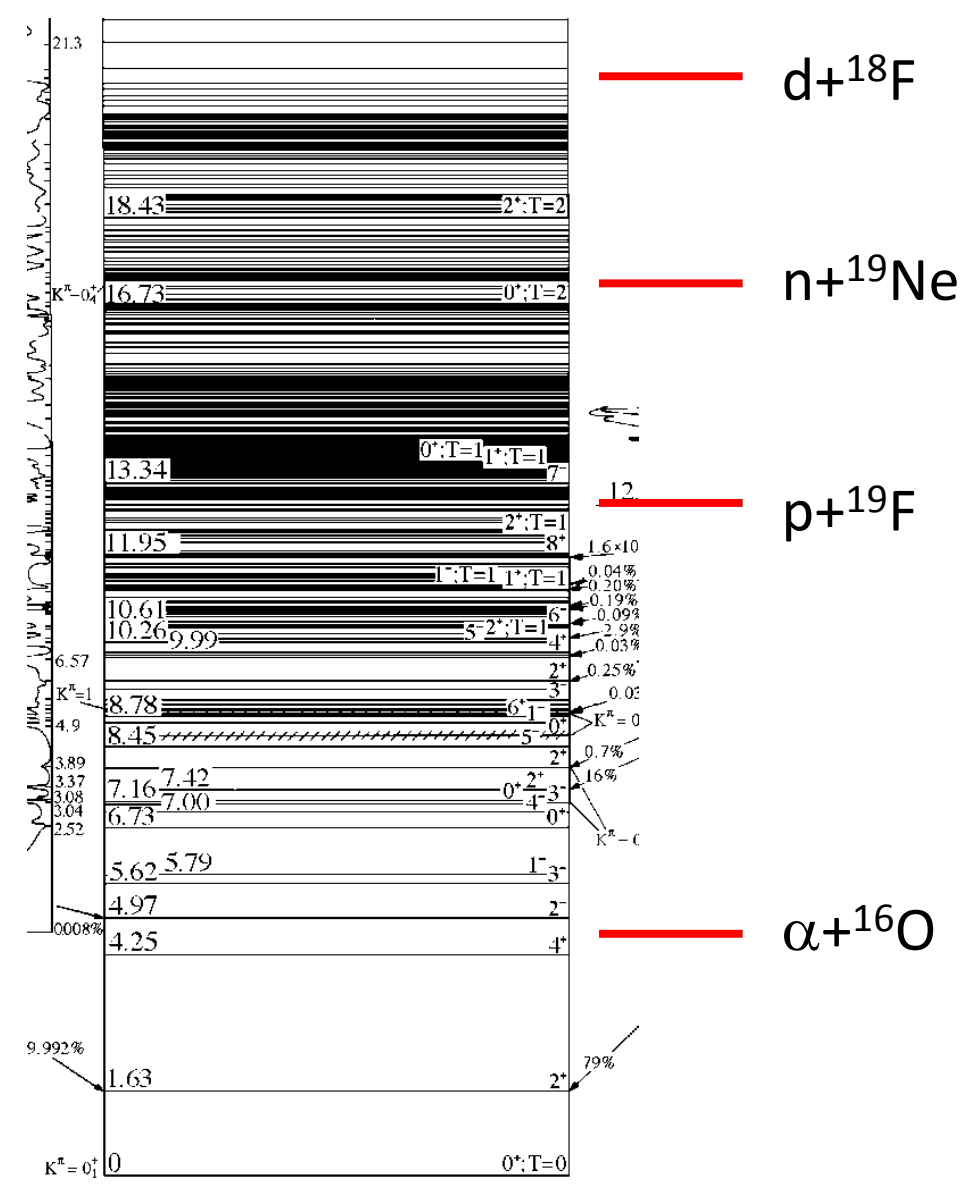

High energies:

- many open channels

- strong absorption

- potential model extended to complex potentials (« optical»)

Phase shift is complex: $\delta=\delta_{R}+i \delta_{I}$

collision matrix: $U=\exp (2 i \delta)=\eta \exp \left(2 i \delta_{R}\right)$

where $\eta=\exp \left(-2 \delta_{I}\right)<1$

Elastic cross section

$$
\frac{d \sigma}{d \Omega}=\frac{1}{4 k^{2}}\left|\sum_{\ell}(2 \ell+1)\left(\eta_{\ell} \exp \left(2 i \delta_{\ell}\right)-1\right) P_{\ell}(\cos \theta)\right|^{2}
$$

Reaction cross section:

$$
\sigma=\frac{\pi}{k^{2}} \sum_{\ell}(2 \ell+1)\left(1-\eta_{\ell}^{2}\right)
$$




\section{Generalizations}

In astrophysics, optical potentials are used to compute fusion cross sections

Fusion cross section: includes many channels

Example: ${ }^{12} \mathrm{C}+{ }^{12} \mathrm{C}$ : Essentially ${ }^{20} \mathrm{Ne}+\alpha,{ }^{23} \mathrm{Na}+\mathrm{p},{ }^{23} \mathrm{Mg}+\mathrm{n}$ channels

$\rightarrow$ absorption simulated by a complex potential $V=V_{R}+i W$

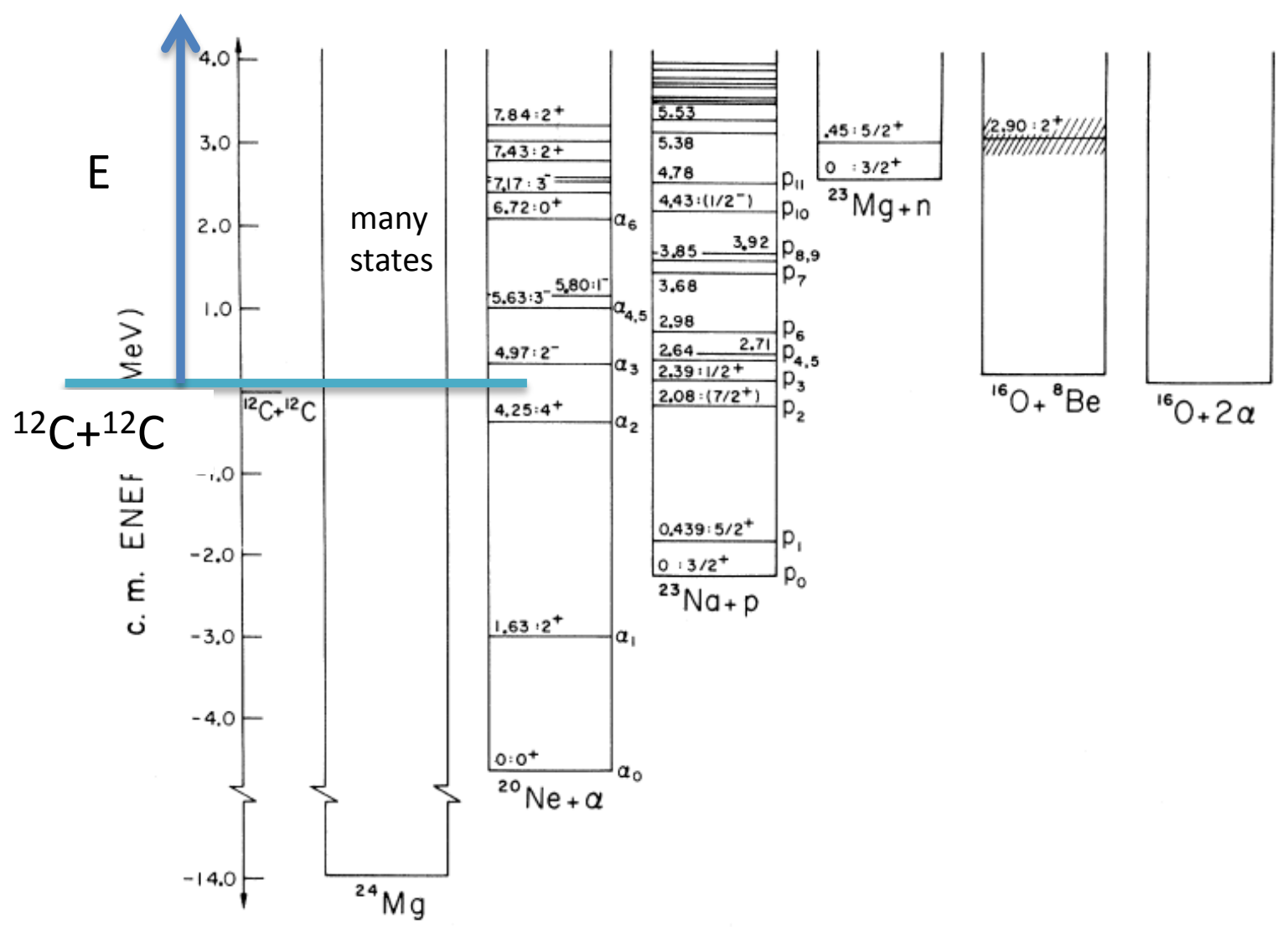




\section{Generalizations}

\section{Typical potentials}

A. Real part

- Woods-Saxon: $V_{R}(r)=-\frac{V_{0}}{1+\exp \left(\frac{r-r_{0}}{a}\right)}$ with parameters $V_{0}, r_{0}, a$ adjusted to experiment

- Folding

$$
V_{R}(r)=\lambda \iint d r_{1} d r_{2} v_{N N}\left(r-r_{1}+r_{2}\right) \rho_{1}\left(r_{1}\right) \rho_{2}\left(r_{2}\right)
$$

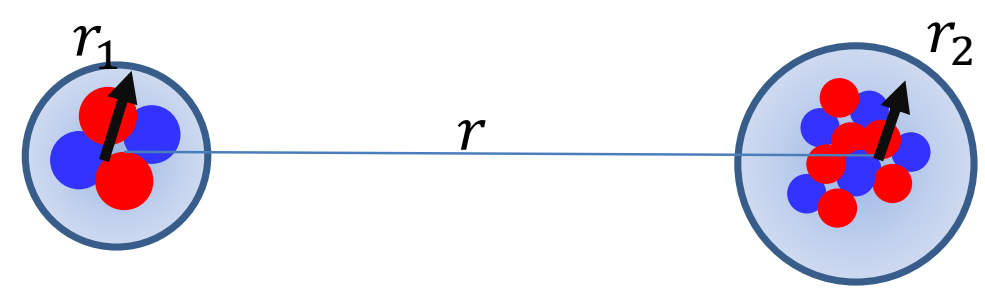

Nucleus 1 density $\rho_{1}\left(r_{1}\right)$

Nucleus 2 density $\rho_{2}\left(r_{2}\right)$

$v_{N N}=$ nucleon-nucleon interaction

$\lambda=$ amplitude $(\sim 1)$, adjustable parameter $\rho_{1}, \rho_{2}=$ nuclear densities (in general known experimentally)

Main advantage: only one parameter $\lambda$ 


\section{Generalizations}

B. Imaginary part

- Woods-Saxon:

Volume: $W(r)=-W_{0} f(r)=-\frac{W_{0}}{1+\exp \left(\frac{r-r_{0}}{a}\right)}$

Surface $W(r)=-W_{0} \frac{d f(r)}{d r}$

- Folding

$W(r)=N_{I} V_{R}(r)$

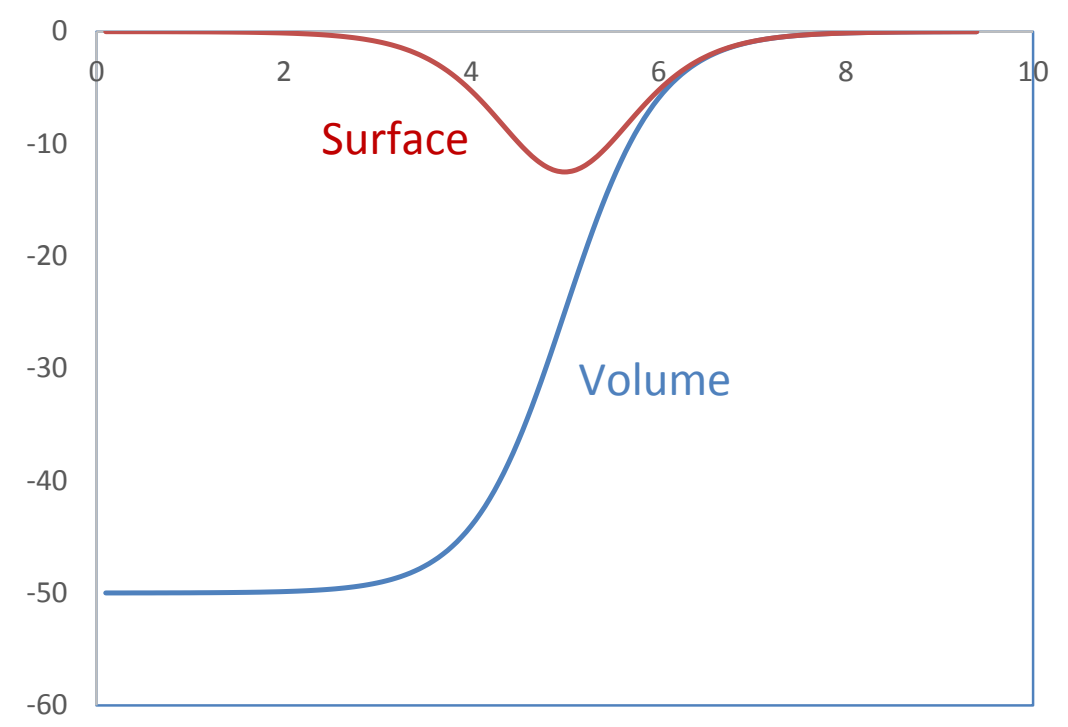




\section{Generalizations}

Example: $\alpha+{ }^{144} \mathrm{Sm}$

P. Mohr et al., Phys. Rev. C55 (1997) 1523

Measurement of elastic scattering $\rightarrow$ optical potential $\rightarrow$ used for astrophysics

$$
\begin{aligned}
& \text { Elastic cross section at } E_{l a b}=20 \mathrm{MeV} \\
& \left(E_{c m}=9.5 \mathrm{MeV}\right)
\end{aligned}
$$
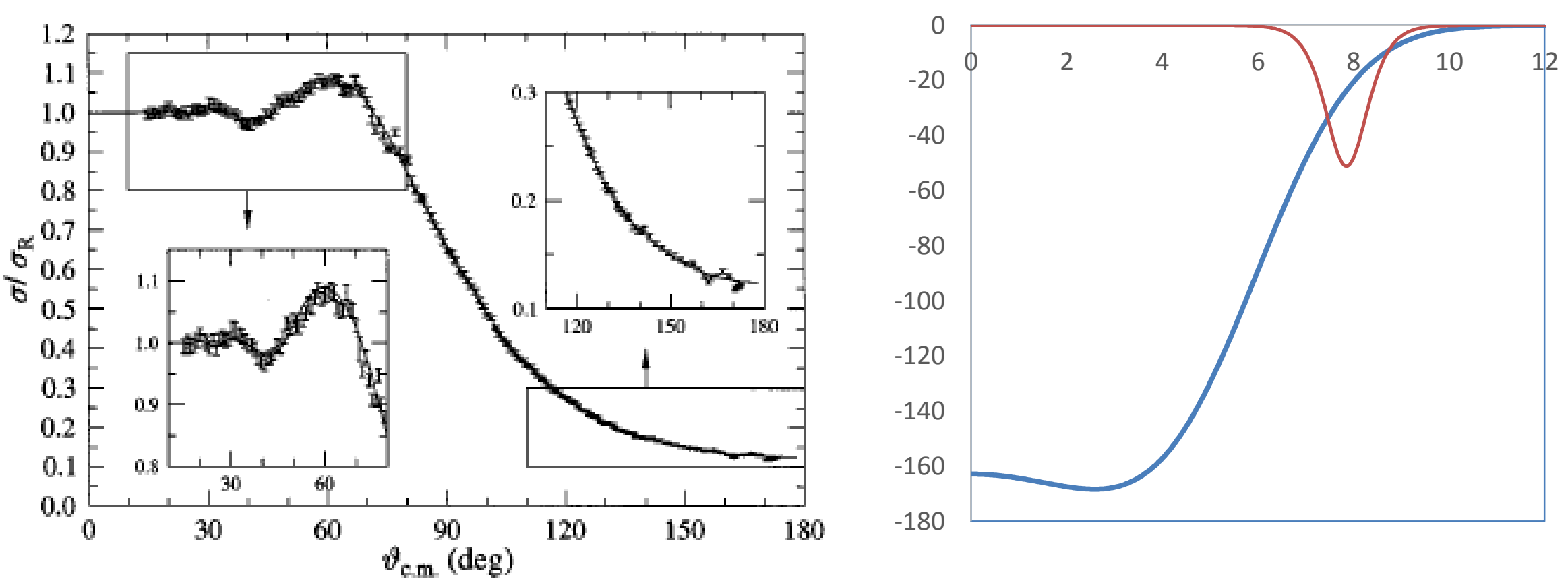
6. Models used for nuclear reactions in astrophysics 


\section{Models used in nuclear astrophysics (for reactions)}

Theoretical methods: Many different cases $\rightarrow$ no "unique" model!

\begin{tabular}{|c|c|c|c|}
\hline Model & Applicable to & Comments & \\
\hline $\begin{array}{l}\text { Potential/optical } \\
\text { model }\end{array}$ & $\begin{array}{l}\text { Capture } \\
\text { Fusion }\end{array}$ & $\begin{array}{l}\text { - Internal structure neglected } \\
\text { - Antisymetrization approximated }\end{array}$ & \\
\hline$R$-matrix & $\begin{array}{l}\text { Capture } \\
\text { Transfer }\end{array}$ & $\begin{array}{l}\text { - No explicit wave functions } \\
\text { - Physics simulated by some parameters }\end{array}$ & $\begin{array}{l}\text { Light } \\
\text { systems }\end{array}$ \\
\hline DWBA & Transfer & $\begin{array}{l}\text { - Perturbation method } \\
\text { - Wave functions in the entrance and exit } \\
\text { channels }\end{array}$ & $\begin{array}{l}\text { Low level } \\
\text { densities }\end{array}$ \\
\hline $\begin{array}{l}\text { Microscopic } \\
\text { models }\end{array}$ & $\begin{array}{l}\text { Capture } \\
\text { Transfer }\end{array}$ & $\begin{array}{l}\text { - Based on a nucleon-nucleon interaction } \\
\text { - A-nucleon problems } \\
\text { - Predictive power }\end{array}$ & \\
\hline $\begin{array}{l}\text { Hauser-Feshbach } \\
\text { Shell model }\end{array}$ & $\begin{array}{l}\text { Capture } \\
\text { Transfer } \\
\text { Capture }\end{array}$ & $\begin{array}{l}\text { - Statistical model } \\
\text { - Only gamma widths }\end{array}$ & $\begin{array}{l}\text { Heavy } \\
\text { systems }\end{array}$ \\
\hline
\end{tabular}


7. Radiative capture in the potential model 


\section{Radiative capture in the potential model}

Potential model: two structureless particles (=optical model, without imaginary part)

- Calculations are simple

- Physics of the problem is identical in other methods

o Spins are neglected

- $\quad \boldsymbol{R}_{\boldsymbol{c m}}=$ center of mass, $\boldsymbol{r}=$ relative coordinate

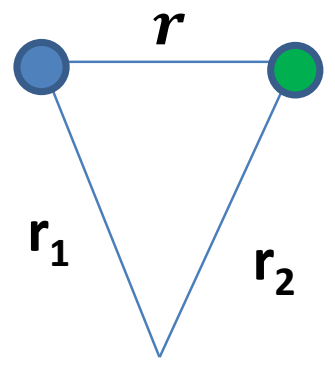

$$
\begin{aligned}
& \boldsymbol{r}_{\mathbf{1}}=\boldsymbol{R}_{\boldsymbol{c m}}-\frac{A_{2}}{A} \boldsymbol{r} \\
& \boldsymbol{r}_{\mathbf{2}}=\boldsymbol{R}_{c m}+\frac{A_{1}}{A} \boldsymbol{r}
\end{aligned}
$$

- Initial wave function:

$\Psi^{\ell_{i} m_{i}}(\boldsymbol{r})=\frac{1}{r} u_{\ell_{i}}(r) Y_{\ell_{i}}^{m_{i}}(\Omega)$, energy $E^{\ell_{i}}=$ scattering energy $E$

Final wave function:

$$
\Psi^{\ell_{f}} m_{f}(\boldsymbol{r})=\frac{1}{r} u_{\ell_{f}}(r) Y_{\ell_{f}}^{m_{f}}(\Omega) \text {, energy } E^{\ell_{f}}
$$

The radial wave functions are given by:

$$
-\frac{\hbar^{2}}{2 \mu}\left(\frac{d^{2}}{d r^{2}}-\frac{\ell(\ell+1)}{r^{2}}\right) u_{\ell}+V(r) u_{\ell}=E^{\ell} u_{\ell}
$$




\section{Radiative capture in the potential model}

- Schrödinger equation: $-\frac{\hbar^{2}}{2 \mu}\left(\frac{d^{2}}{d r^{2}}-\frac{\ell(\ell+1)}{r^{2}}\right) u_{\ell}+V(r) u_{\ell}=E^{\ell} u_{\ell}$

- Typical potentials:

- coulomb =point-sphere

- nuclear: Woods-Saxon, Gaussian parameters adjusted on important properties (bound-state energy, phase shifts, etc.)

- Potentials can be different in the initial and final states

- Wave functions computed numerically (Numerov algorithm)

- Limitations

- initial (scattering state): must reproduce resonances (if any)

- final (bound) state: must have a A+B structure

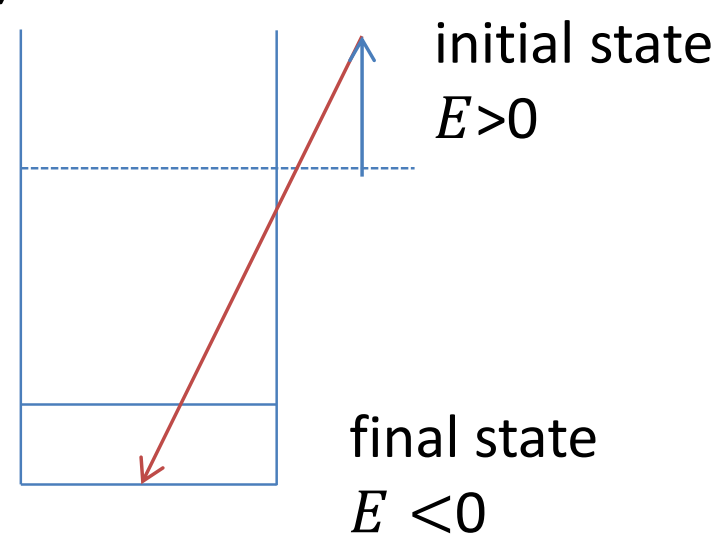




\section{Radiative capture in the potential model}

Some typical examples
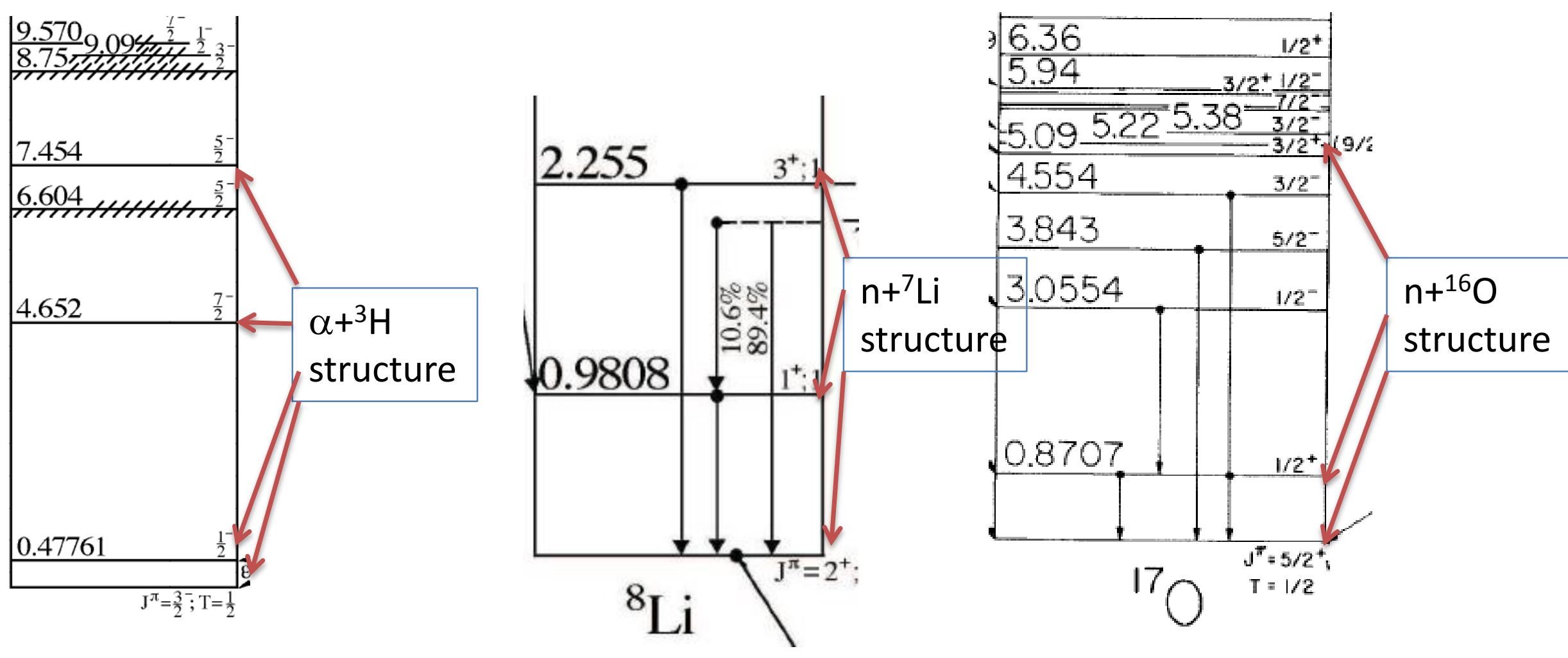

${ }^{7} \mathrm{Li}$

Problem more and more important when the level density increases

$\rightarrow$ in practice: limited to low-level densities (light nuclei or nuclei close to the drip lines) 


\section{Radiative capture in the potential model}

- Electric operator for two particles:

$\mathcal{M}_{\mu}^{E \lambda}=e\left(Z_{1}\left|\boldsymbol{r}_{\mathbf{1}}-\boldsymbol{R}_{\boldsymbol{c m}}\right|^{\lambda} Y_{\lambda}^{\mu}\left(\Omega_{r_{1}-R_{c m}}\right)+Z_{2}\left|\boldsymbol{r}_{\mathbf{2}}-\boldsymbol{R}_{\boldsymbol{c m}}\right|^{\lambda} Y_{\lambda}^{\mu}\left(\Omega_{r_{2}-R_{c m}}\right)\right)$

which provides

$$
\mathcal{M}_{\mu}^{E \lambda}=e\left[Z_{1}\left(-\frac{A_{2}}{A}\right)^{\lambda}+Z_{2}\left(\frac{A_{1}}{A}\right)^{\lambda}\right] r^{\lambda} Y_{\lambda}^{\mu}\left(\Omega_{r}\right)=e Z_{e f f} r^{\lambda} Y_{\lambda}^{\mu}\left(\Omega_{r}\right)
$$

- Matrix elements needed for electromagnetic transitions

$$
<\Psi^{J_{f} m_{f}}\left|\mathcal{M}_{\mu}^{E \lambda}\right| \Psi^{J_{i} m_{i}}>=e Z_{\text {eff }}<Y_{J_{f}}^{m_{f}}\left|Y_{\lambda}^{\mu}\right| Y_{J_{i}}^{m_{i}}>\int_{0}^{\infty} u_{J_{i}}(r) u_{J_{f}}(r) r^{\lambda} d r
$$

- Reduced matrix elements:

$\left.<\Psi^{J_{f}}\left\|\mathcal{M}^{E \lambda}\right\| \Psi^{J_{i}}\right\rangle=e Z_{\text {eff }}<J_{f} 0 \lambda 0\left|J_{i} 0\right\rangle$

$$
\times\left(\frac{\left(2 J_{i}+1\right)(2 \lambda+1)}{4 \pi\left(2 J_{f}+1\right)}\right)^{1 / 2} \int_{0}^{\infty} u_{J_{i}}(r) u_{J_{f}}(r) r^{\lambda} d r
$$

$\rightarrow$ simple one-dimensional integrals 


\section{Radiative capture in the potential model}

\section{Assumptions:}

- spins zero: $\ell_{i}=J_{i}, \ell_{f}=J_{f}$

- given values of $J_{i}, J_{f}, \lambda$

Integrated cross section

$$
\sigma_{\lambda}(E)=\frac{8 \pi}{k^{2}} \frac{e^{2}}{\hbar c} Z_{e f f}^{2} k_{\gamma}^{2 \lambda+1} F\left(\lambda, J_{i}, J_{f}\right)\left|\int_{0}^{\infty} u_{J_{i}}(r, E) u_{J_{f}}(r) r^{\lambda} d r\right|^{2}
$$

with

- $Z_{\text {eff }}=Z_{1}\left(-\frac{A_{2}}{A}\right)^{\lambda}+Z_{2}\left(\frac{A_{1}}{A}\right)^{\lambda}$

- $F\left(\lambda, J_{i}, J_{f}\right)=<J_{i} \lambda 00 \mid J_{f} 0>\left(2 J_{i}+1\right) \frac{(\lambda+1)(2 \lambda+1)}{\lambda(2 \lambda+1) ! !^{2}}$

- $k_{\gamma}=\frac{E-E_{f}}{\hbar c}$

Normalization

- final state (bound): normalized to unity $u_{J}(r) \rightarrow C W\left(2 k_{B} r\right) \rightarrow C \exp \left(-k_{B} r\right)$

- initial state (continuum): $u_{J}(r) \rightarrow F_{J}(k r) \cos \delta_{J}+G_{J}(k r) \sin \delta_{J}$ 


\section{Radiative capture in the potential model}

Integrated vs differential cross sections

- Total (integrated) cross section:

$$
\sigma(E)=\sum_{\lambda} \sigma_{\lambda}(E)
$$

$\rightarrow$ no interference between the multipolarities

- Differential cross section:

- $P_{\lambda}(\theta)=$ Legendre polynomial

$$
\frac{d \sigma}{d \theta}=\left|\sum_{\lambda} a_{\lambda}(E) P_{\lambda}(\theta)\right|^{2}
$$

- $a_{\lambda}(E)$ are complex, $\sigma_{\lambda}(E) \sim\left|a_{\lambda}(E)\right|^{2}$

$\rightarrow$ interference effects

$\rightarrow$ angular distributions are necessary to separate the multipolarities

$\rightarrow$ in general one multipolarity is dominant (not in ${ }^{12} \mathrm{C}(\alpha, \gamma){ }^{16} \mathrm{O}$ : E1 and E2) 


\section{Radiative capture in the potential model}

\section{Example: ${ }^{12} \mathrm{C}(\mathrm{p}, \gamma){ }^{13} \mathrm{~N}$}

- First reaction of the CNO cycle

- Well known experimentally

- Presents a low energy resonance $\left(\ell=0 \rightarrow J=1 / 2^{+}\right)$

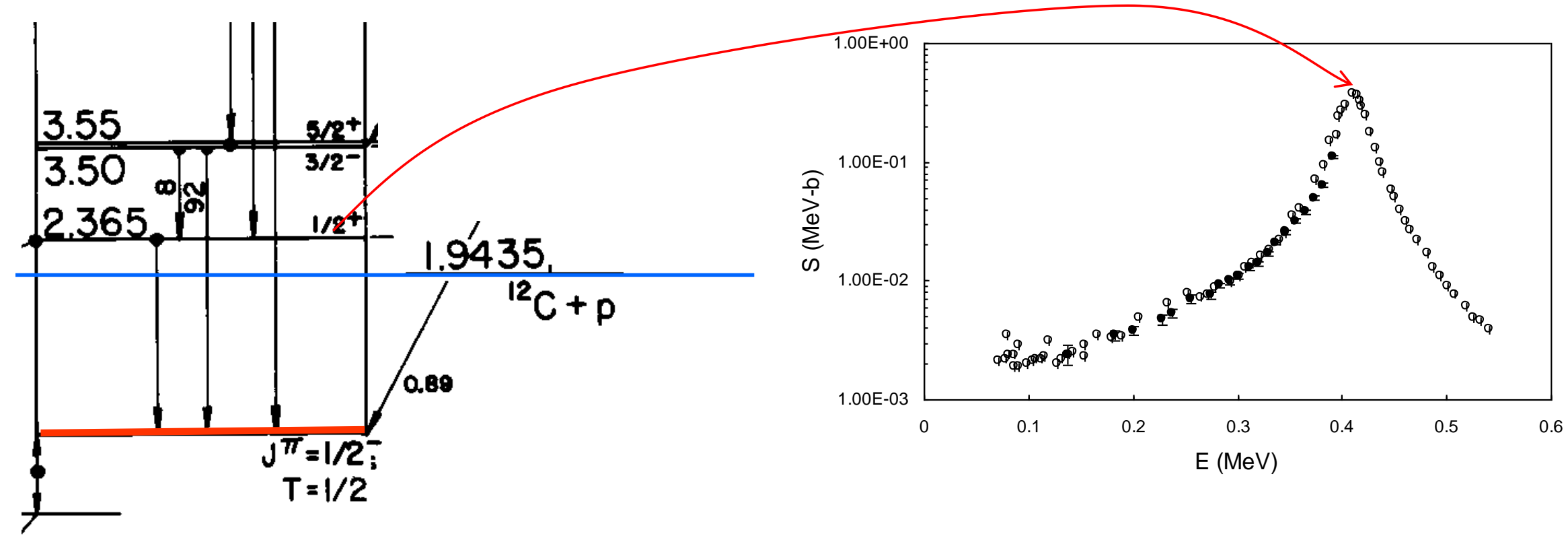

$$
\begin{aligned}
& \text { Potential : } V=-55.3^{*} \exp \left(-(r / 2.70)^{2}\right) \quad \text { (final state) } \\
& -70.5^{\star} \exp \left(-(r / 2.70)^{2}\right) \quad \text { (initial state) }
\end{aligned}
$$




\section{Radiative capture in the potential model}

Final state: $J_{f}=1 / 2^{-}$

Initial state: $\ell_{i}=0 \rightarrow J_{i}=1 / 2^{+}$

$\rightarrow$ E1 transition $1 / 2^{+} \rightarrow 1 / 2^{-}$

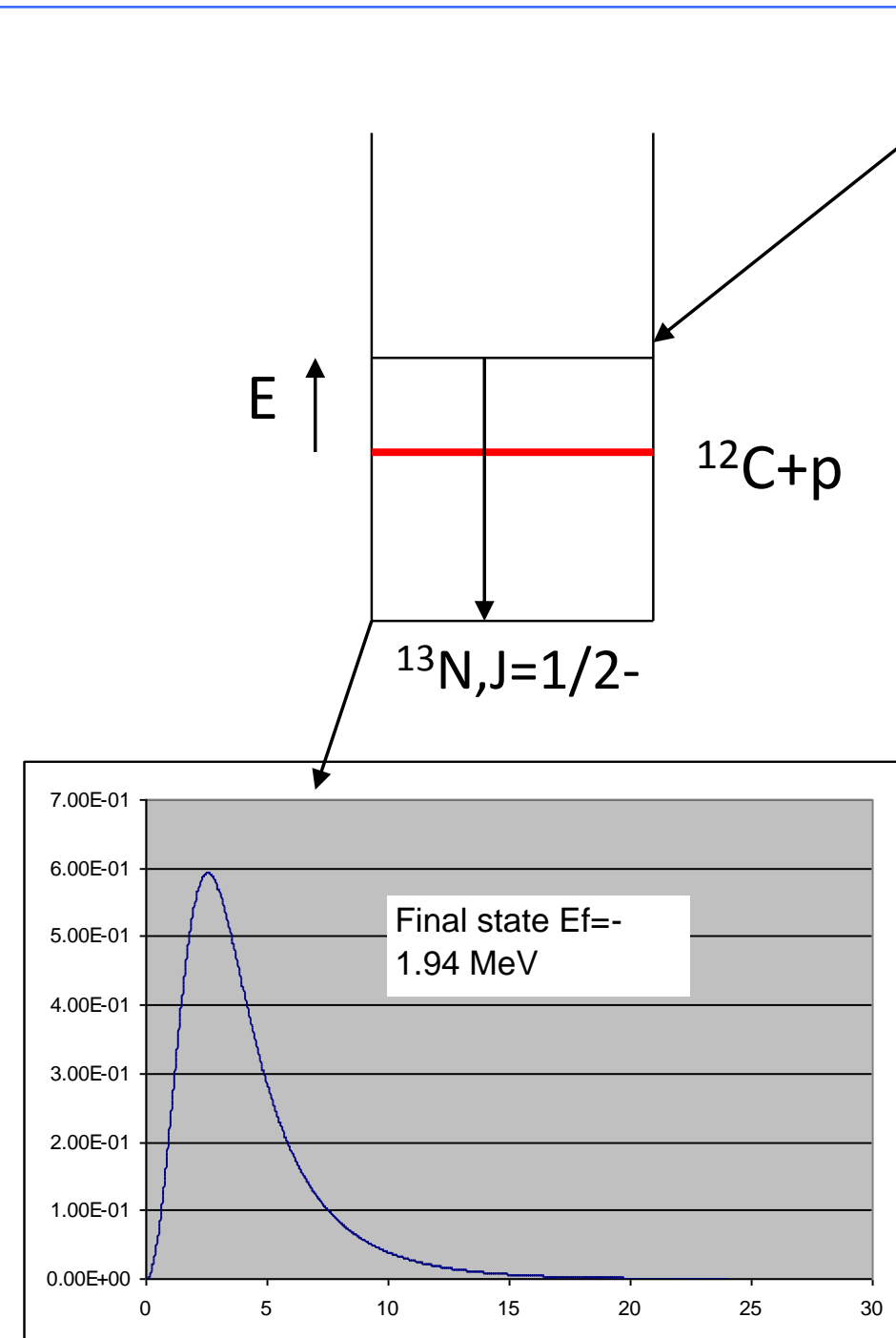

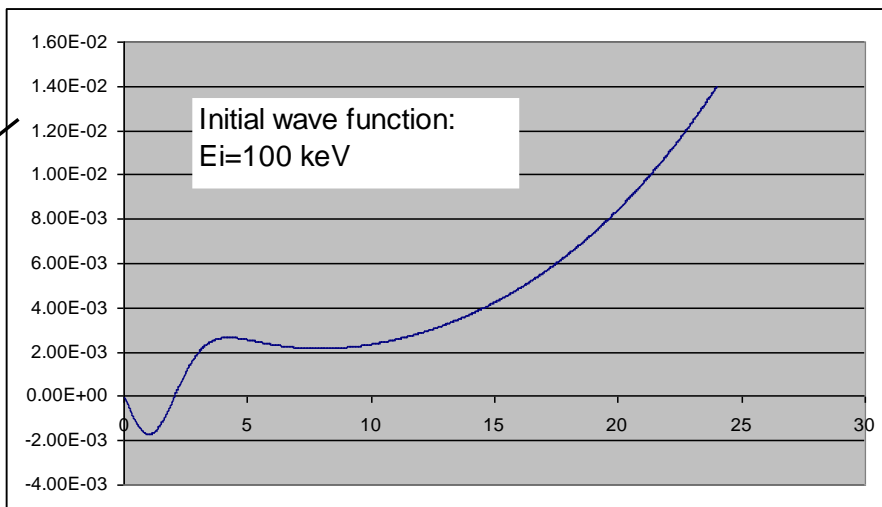
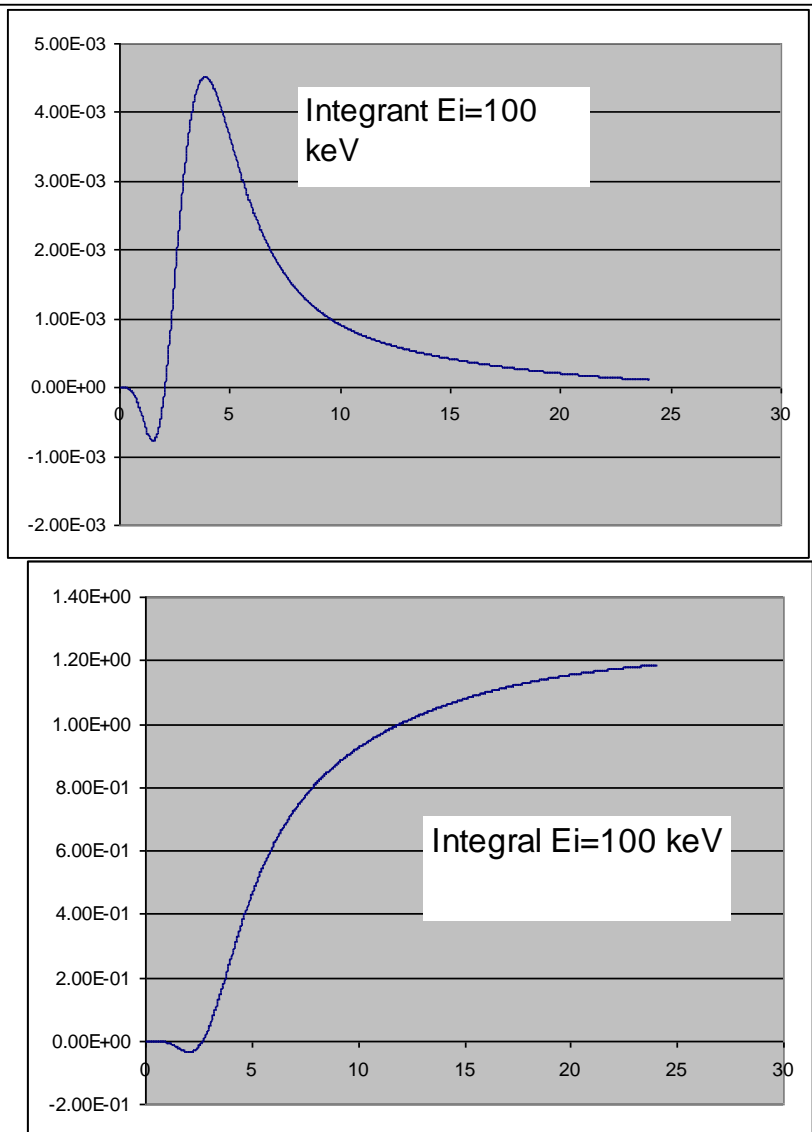


\section{Radiative capture in the potential model}

The calculation is repeated at all energies

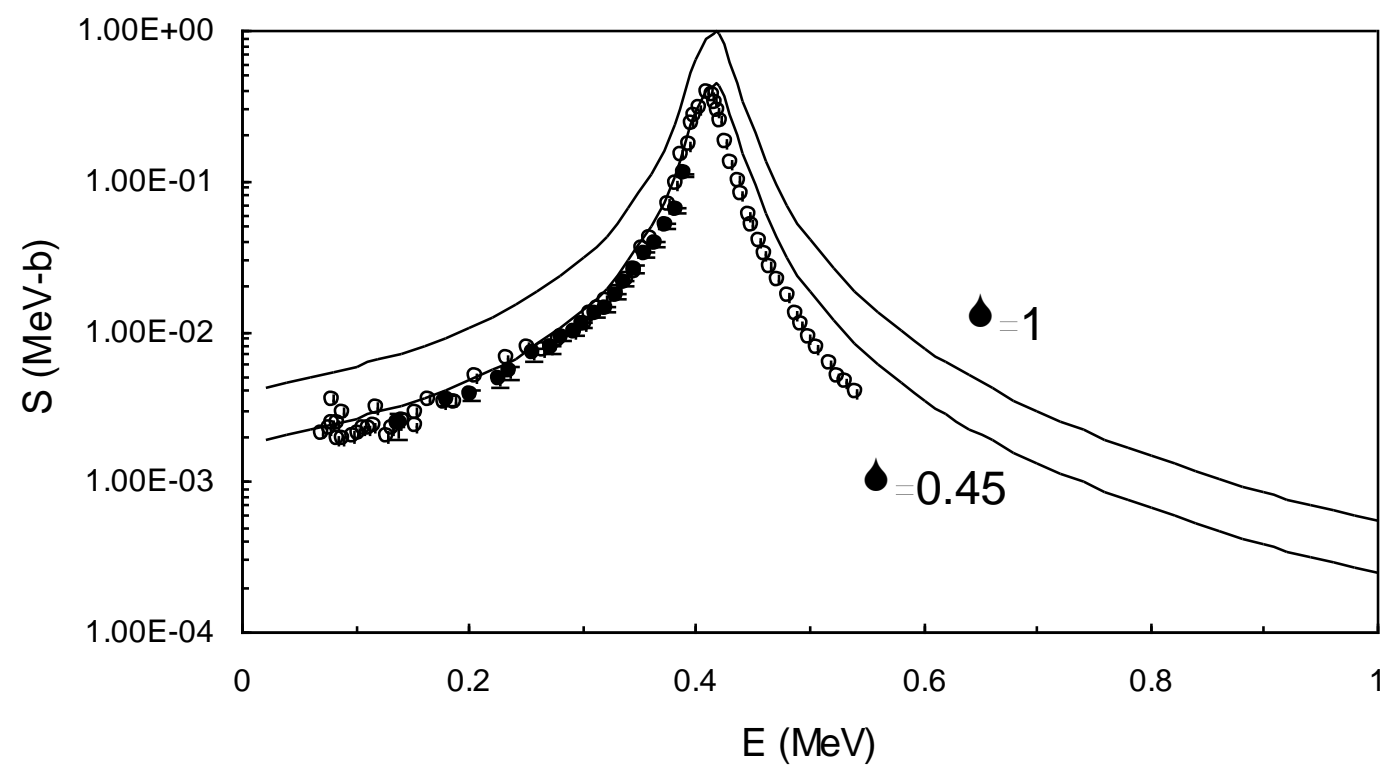

Necessity of a spectroscopic factor $S$

Assumption of the potential model: ${ }^{13} \mathrm{~N}={ }^{12} \mathrm{C}+\mathrm{p}$

In reality ${ }^{13} \mathrm{~N}={ }^{12} \mathrm{C}+\mathrm{p} \oplus{ }^{12} \mathrm{C}^{*}+\mathrm{p} \oplus{ }^{9} \mathrm{Be}+\alpha \oplus \ldots$

$\rightarrow$ to simulate the missing channels: $u_{f}(r)$ is replaced by $S^{1 / 2} u_{f}(r)$ $S=$ spectroscopic factor

Other applications: ${ }^{7} \mathrm{Be}(\mathrm{p}, \gamma)^{8} \mathrm{~B},{ }^{3} \mathrm{He}(\alpha, \gamma)^{7} \mathrm{Be}$, etc... 
8. The R-matrix method

- General presentation

- Single resonance system

- Applications to elastic scattering ${ }^{12} \mathrm{C}+\mathrm{p}$

- Application to ${ }^{12} \mathrm{C}(p, \gamma){ }^{13} \mathrm{~N}$ and ${ }^{12} \mathrm{C}(\alpha, \gamma){ }^{16} \mathrm{O}$ 


\section{The R-matrix method}

- Introduced by Wigner (1937) to parametrize resonances (nuclear physics) In nuclear astrophysics: used to fit data

- Provides scattering properties at all energies (not only at resonances)

- Based on the existence of 2 regions (radius a):

- Internal: coulomb+nuclear

- external: coulomb

Exit channels

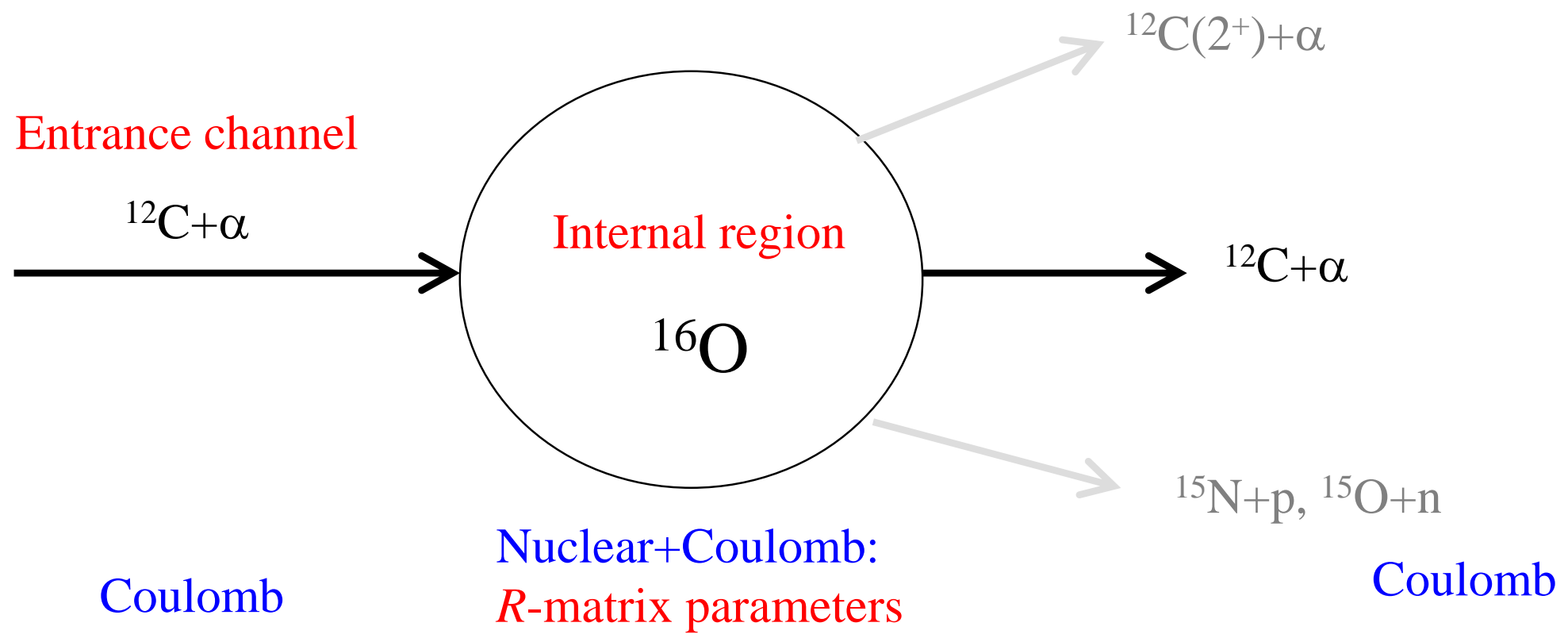




\section{The R-matrix method}

Main Goal: fit of experimental data
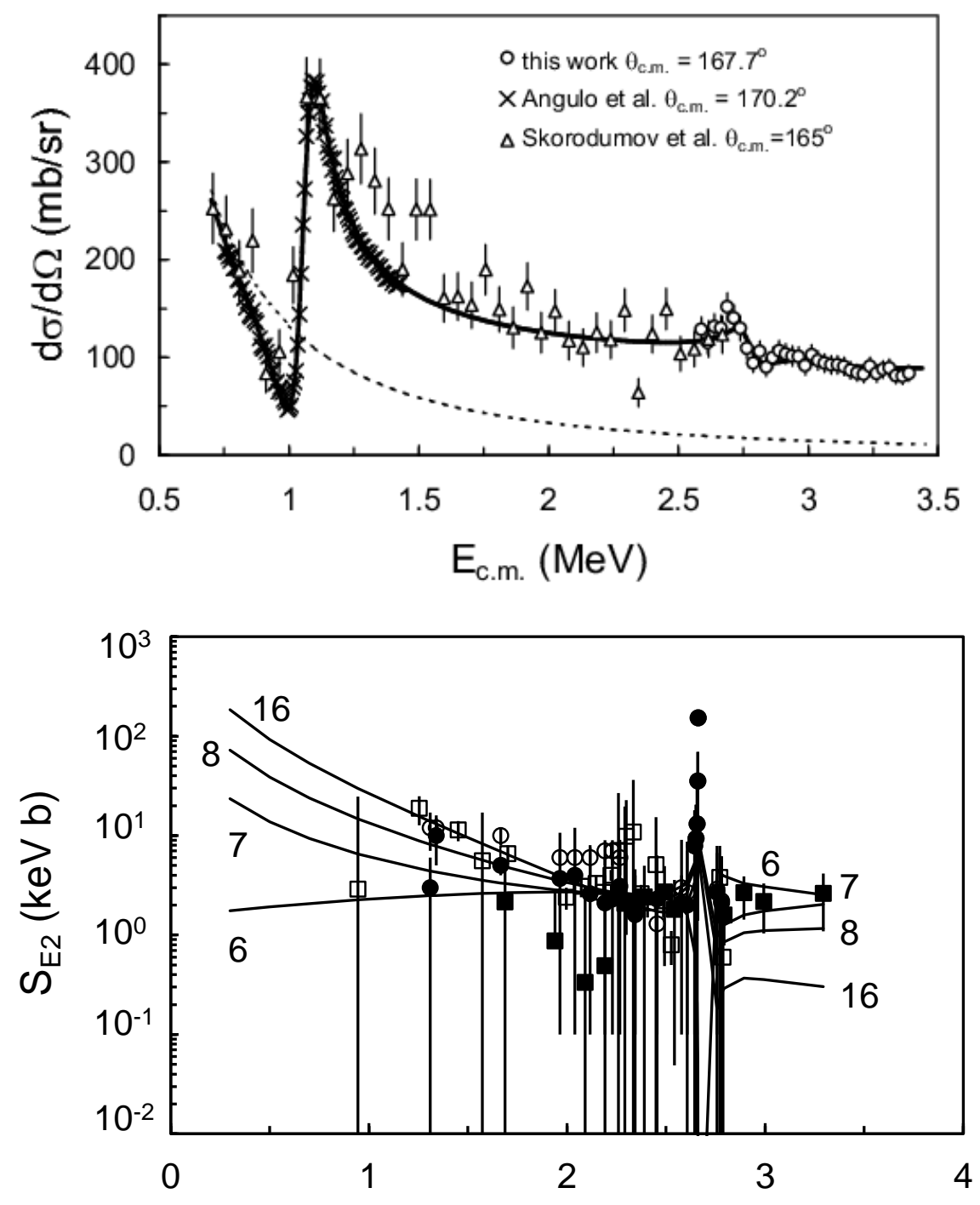

${ }^{18} \mathrm{Ne}+\mathrm{p}$ elastic scattering

$\rightarrow$ resonance properties
Nuclear astrophysics: ${ }^{12} \mathrm{C}(\alpha, \gamma)^{16} \mathrm{O}$

$\rightarrow$ Extrapolation to low energies 


\section{The R-matrix method}

- Internal region: The $\mathrm{R}$ matrix is given by a set of resonance parameters $E_{i}, \gamma_{i}^{2}$

$$
R(E)=\sum_{i} \frac{\gamma_{i}^{2}}{E_{i}-E}=a \frac{\Psi^{\prime}(a)}{\Psi(a)}\left|\begin{array}{l}
\mathrm{i}=3, E_{3}, \gamma_{3}^{2} \\
\mathrm{i}=2, E_{2}, \gamma_{2}^{2} \\
\cline { 2 - 2 }
\end{array}\right| \begin{aligned}
& \mathrm{i}=1, E_{1}, \gamma_{1}^{2}
\end{aligned}
$$

- External region: Coulomb behaviour of the wave function

$$
\Psi(r)=I(r)-U O(r)
$$

$\rightarrow$ the collision matrix $U$ is deduced from the R-matrix (repeated for each spin/parity $J \pi$ )

- Two types of applications:

- phenomenological R matrix: $\gamma_{i}^{2}$ and $E_{i}$ are fitted to the data (astrophysics)

- calculable R matrix: $\gamma_{i}^{2}$ and $E_{i}$ are computed from basis functions (scattering theory)

- R-matrix radius $a$ is not a parameter: the cross sections must be insensitive to $a$

- Can be extended to multichannel calculations (transfer), capture, etc.

- Well adapted to nuclear astrophysics: low energies, low level densities 


\section{The R-matrix method}

A simple case: elastic scattering with a single isolated resonance

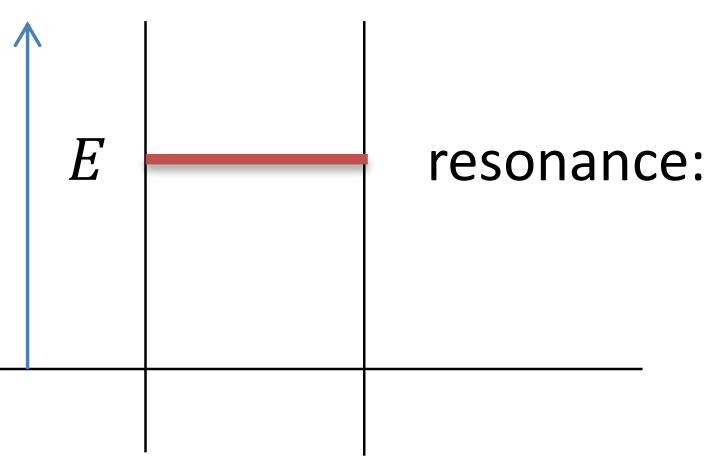

threshold

- From the total width $\Gamma \rightarrow$ reduced width $\Gamma=2 \gamma^{2} P_{l}\left(E_{R}\right)$

$P_{l}\left(E_{R}\right)=$ penetration factor

- Link between $\left(E_{R}, \gamma^{2}\right) \leftrightarrow\left(E_{0}, \gamma_{0}^{2}\right)$

- Calculation of the R-matrix $R(E)=\frac{\gamma_{0}^{2}}{E_{0}-E}$

- Calculation of the scattering matrix: $U(E)=\frac{I(k a)}{O(k a)} \frac{1-L^{*} R(E)}{1-L R(E)}$ (must be done for each $\ell$ )

- Calculation of the cross section $\rightarrow E_{0}$ and/or $\gamma_{0}^{2}$ can be fitted 


\section{The R-matrix method}

\section{Example: ${ }^{12} \mathrm{C}+\mathrm{p}: \mathrm{E}_{\mathrm{R}}=0.42 \mathrm{MeV}$}

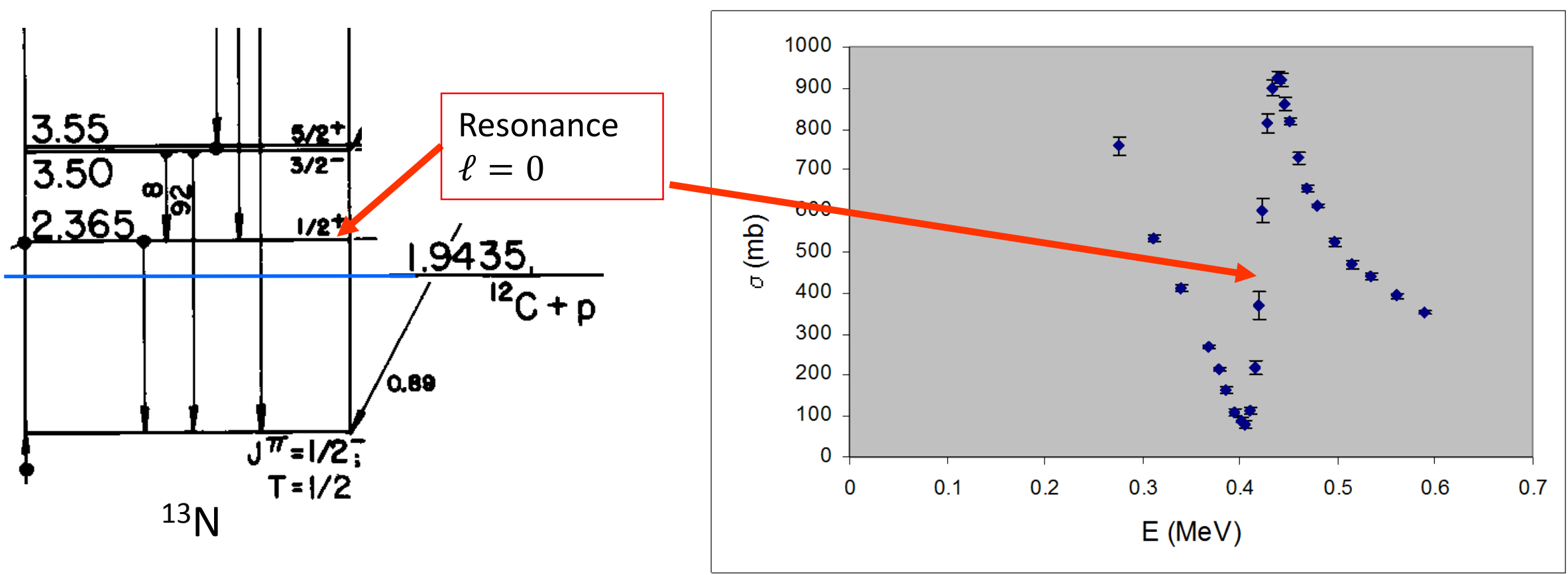

In the considered energy range: resonance $\mathrm{J}=1 / 2+(\ell=0)$

$\rightarrow$ Phase shift for $\ell=0$ is treated by the $R$ matrix

$\rightarrow$ Other phase shifts $\ell>0$ are given by the hard-sphere approximation 


\section{The R-matrix method}

First example: Elastic scattering ${ }^{12} \mathrm{C}+\mathrm{p}$

Data from H.O. Meyer et al., Z. Phys. A279 (1976) 41
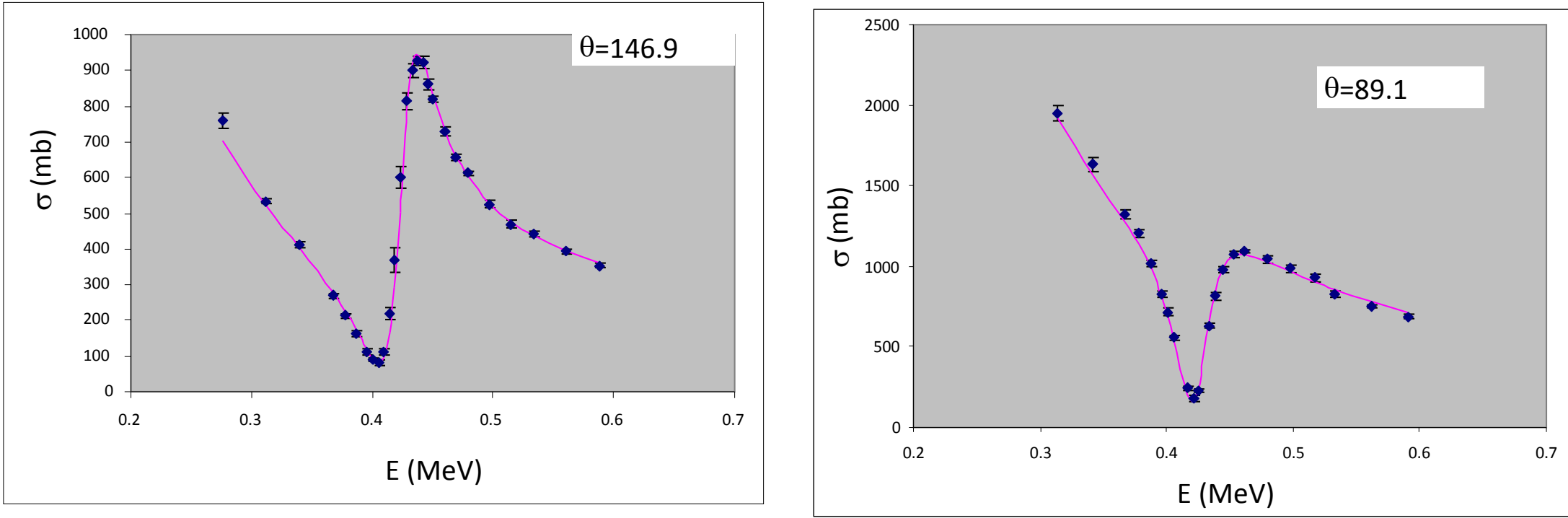

$\mathrm{R}$ matrix fits for different channel radii

\begin{tabular}{|c|c|c|c|c|c|}
\hline $\mathrm{a}$ & $\mathrm{E}_{\mathrm{R}}$ & $\Gamma$ & $\mathrm{E}_{0}$ & $\gamma_{0} 2$ & $\chi^{2}$ \\
\hline 4.5 & 0.4273 & 0.0341 & -1.108 & 1.334 & 2.338 \\
\hline 5 & 0.4272 & 0.0340 & -0.586 & 1.068 & 2.325 \\
\hline 5.5 & 0.4272 & 0.0338 & -0.279 & 0.882 & 2.321 \\
\hline 6 & 0.4271 & 0.0336 & -0.085 & 0.745 & 2.346 \\
\hline
\end{tabular}

$\rightarrow E_{R}, \Gamma$ very stable with $a$

$\rightarrow$ global fit independent of $a$ 


\section{The R-matrix method}

Extension to transfer, example: ${ }^{18} \mathrm{~F}(p, \alpha){ }^{15} \mathrm{O}$

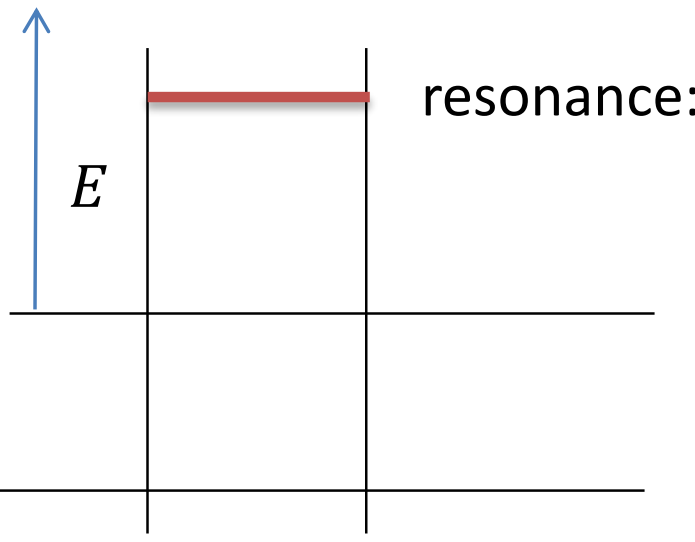

threshold $2\left(\alpha+{ }^{15} \mathrm{O}\right)$

- Link between $\left(E_{R}, \gamma_{1}^{2}, \gamma_{2}^{2}\right) \leftrightarrow E_{0}, \gamma_{01}^{2}, \gamma_{02}^{2}$ more complicated

- R-matrix: $2 \times 2$ matrix

$$
\begin{array}{ll}
R_{i i}(E)=\frac{\gamma_{01}^{2}}{E_{0}-E} & \text { associated with the entrance channel } \\
R_{f f}(E)=\frac{\gamma_{02}^{2}}{E_{0}-E} & \text { associated with the exit channel } \\
R_{i f}(E)=\frac{\gamma_{01} \gamma_{02}}{E_{0}-E} & \text { associated with the transfer }
\end{array}
$$

- Scattering matrix: $2 \times 2$ : $U_{11}, U_{22} \rightarrow$ elastic cross sections

$U_{12}, \rightarrow$ transfer cross section

- More parameters, but some are common to elastic scattering $\left(E_{0}, \gamma_{01}^{2}\right)$

$\rightarrow$ constraints with elastic scattering 


\section{The R-matrix method}

Recent application to ${ }^{18} \mathrm{~F}(\mathrm{p}, \mathrm{p})^{18} \mathrm{~F}$ and ${ }^{18} \mathrm{~F}(\mathrm{p}, \alpha)^{15} \mathrm{O}$

D. Mountford et al, Phys. Rev. C 85 (2012) 022801

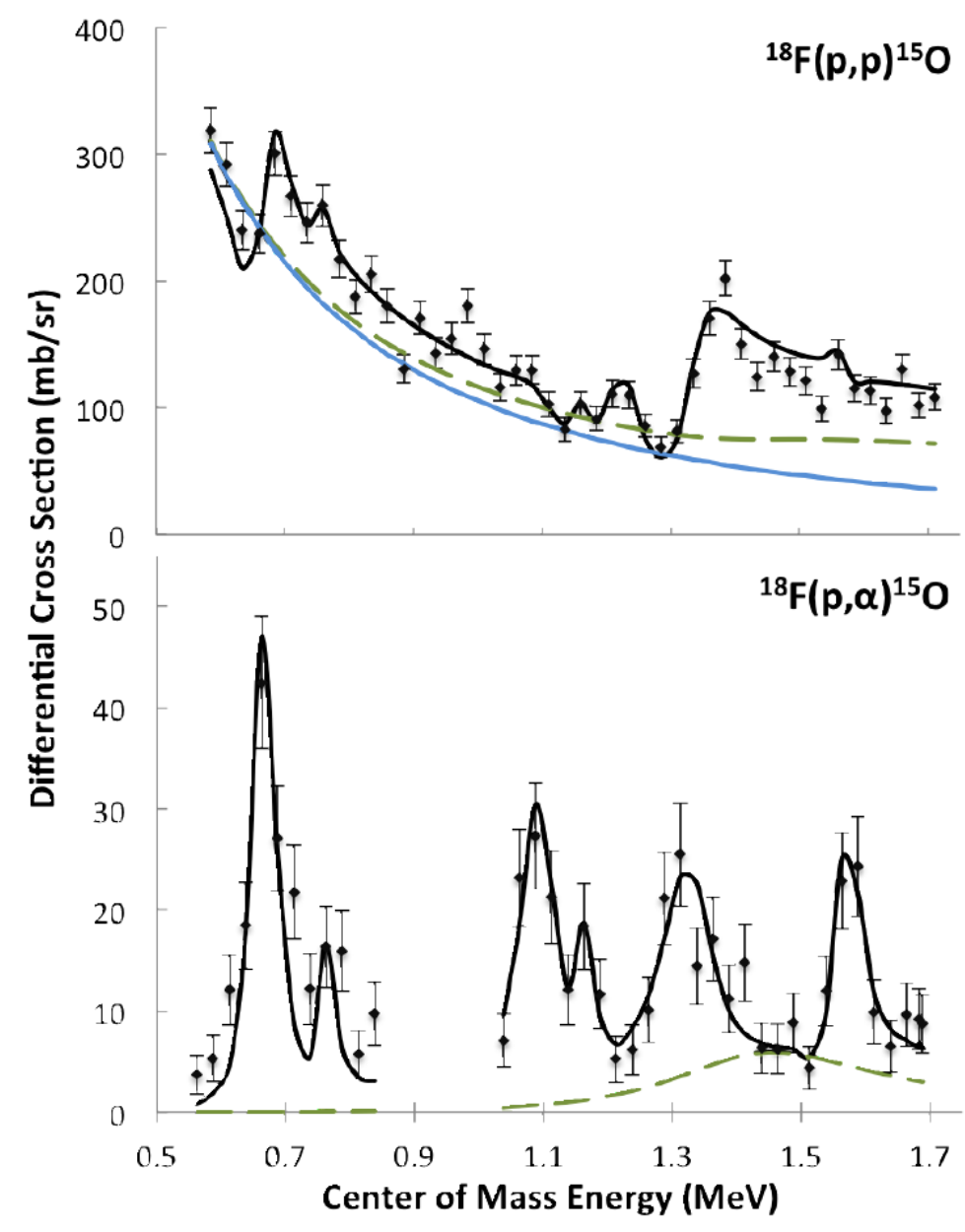

simultaneous fit of both cross sections angle: $176^{\circ}$

for each resonance: $J \pi, E_{R}, \Gamma_{p}, \Gamma_{\alpha}$ 8 resonances $\rightarrow 24$ parameters 


\section{The R-matrix method}

Extension to radiative capture

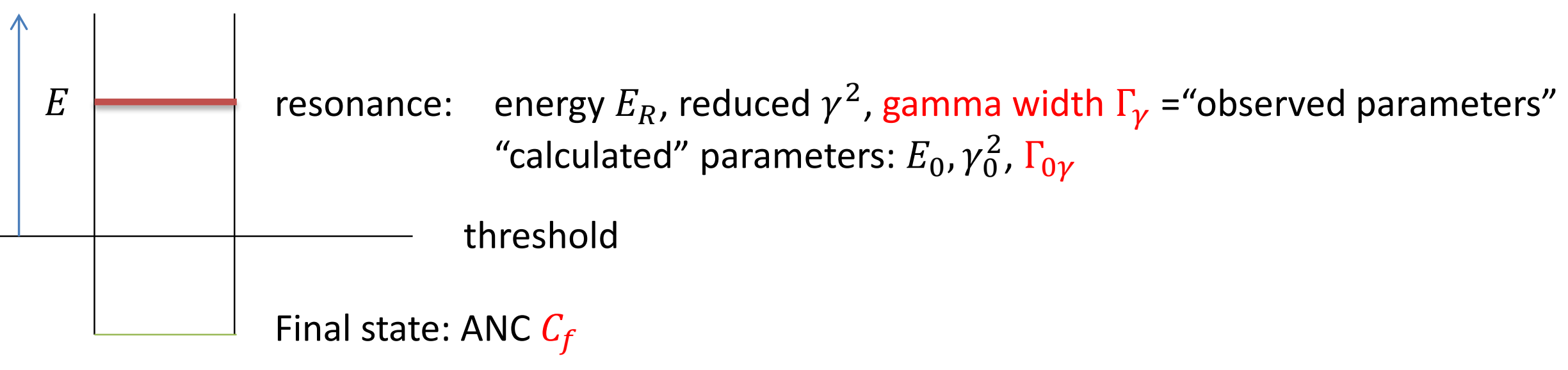

Capture reaction $=$ transition between an initial state at energy $E$ to bound states

Cross section $\sigma_{C}(E) \sim\left|<\Psi_{f}\right| H_{\gamma}\left|\Psi_{i}(E)>\right|^{2}$

Additional pole parameter: gamma width $\Gamma_{\gamma i}$

$<\Psi_{f}\left|H_{\gamma}\right| \Psi_{i}(E)>=<\Psi_{f}\left|H_{\gamma}\right| \Psi_{i}(E)>_{i n t}+<\Psi_{f}\left|H_{\gamma}\right| \Psi_{i}(E)>_{\text {ext }}$

internal part: $<\Psi_{f}\left|H_{\gamma}\right| \Psi_{i}(E)>_{i n t} \sim \sum_{i=1}^{N} \frac{\gamma_{i} \sqrt{\Gamma_{\gamma i}}}{E_{i}-E}$

external part: $<\Psi_{f}\left|H_{\gamma}\right| \Psi_{i}(E)>_{e x t} \sim C_{f} \int_{a}^{\infty} W\left(2 k_{f} r\right) r^{\lambda}\left(I_{i}(k r)-U O_{i}(k r)\right) d r$ 


\section{The R-matrix method}

External part: $\left\langle\Psi_{f}\left|H_{\gamma}\right| \Psi_{i}(E)>_{\text {ext }} \sim C_{f} \int_{a}^{\infty} W\left(2 k_{f} r\right) r^{\lambda}\left(I_{i}(k r)-U O_{i}(k r)\right) d r\right.$

Essentially depends on $k_{f}$

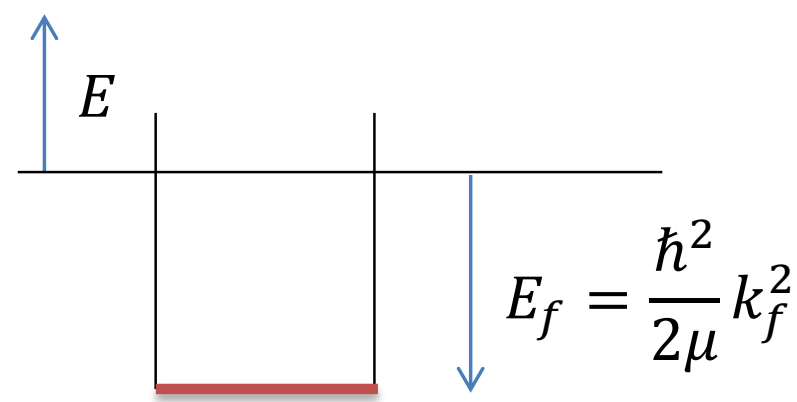

Witthaker function $W\left(2 k_{f} r\right) \sim \exp \left(-k_{f} r\right)$

- $k_{f}$ large: fast decrease example ${ }^{12} \mathrm{C}(\alpha, \gamma){ }^{16} \mathrm{O}, E_{f}=7.16 \mathrm{MeV}, \mu=3 \quad \rightarrow$ external term negligible $\rightarrow$ insensitive to $C_{f}$

- $k_{f}$ small: slow decrease example: ${ }^{7} \mathrm{Be}(\mathrm{p}, \gamma)^{8} \mathrm{~B}, E_{f}=0.137 \mathrm{MeV}, \mu=7 / 8 \rightarrow$ external term dominant $\rightarrow$ mainly given by $C_{f}$

- Contribution of internal/external terms depends on energy (external larger at low energies) 


\section{The R-matrix method}

Example 1: $\left.{ }^{12} \mathrm{C}(\mathrm{p}, \gamma)\right)^{13} \mathrm{~N}$ : R-matrix calculation with a single pole

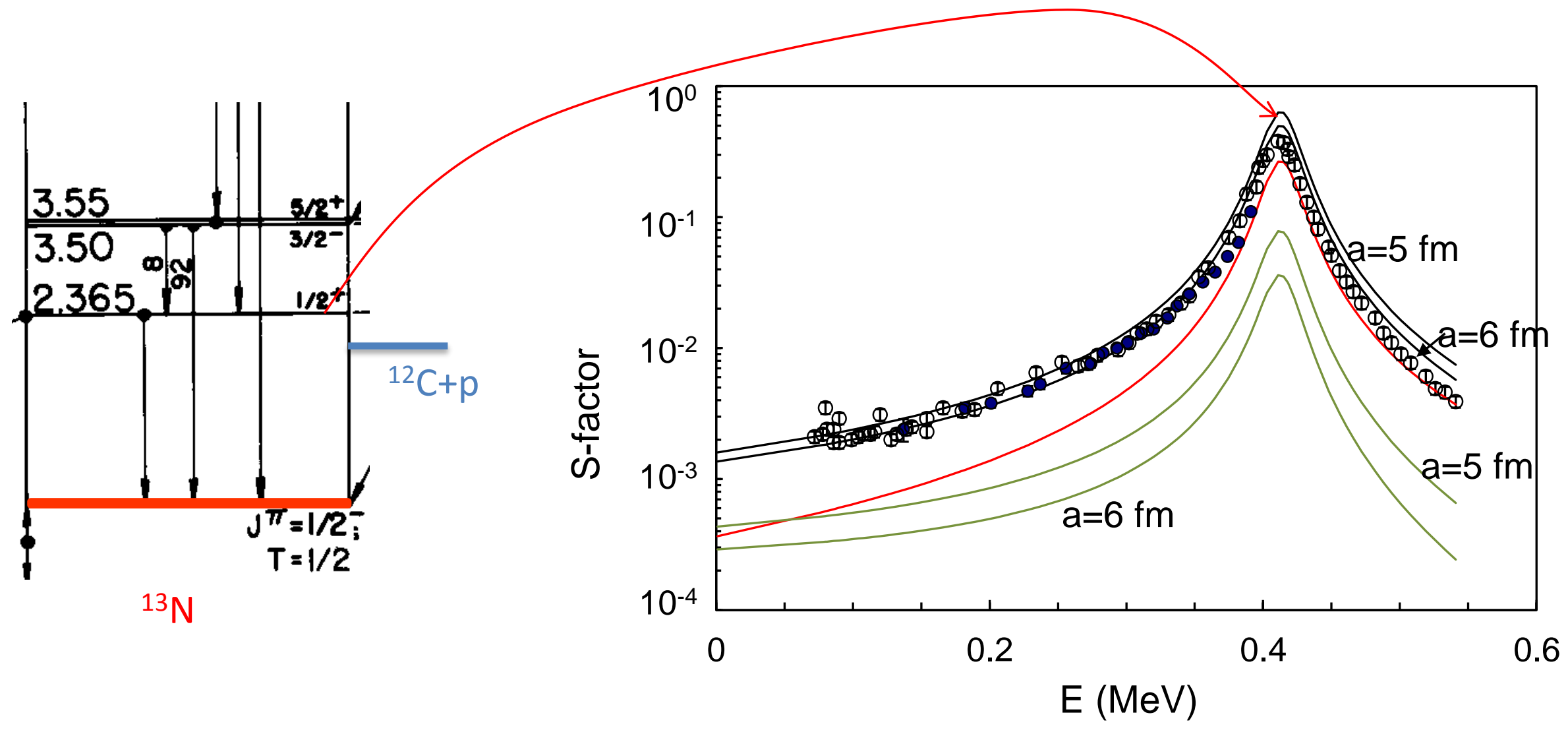

Experiment: $E_{R}=0.42 \mathrm{MeV}, \Gamma_{p}=31 \mathrm{keV}, \Gamma_{\gamma}=0.4 \mathrm{eV}$

Red line: internal contribution, pure Breit-Wigner approximation

Green lines: external contribution: important at low energies, sensitive to the ANC 


\section{The R-matrix method}

Example 2: ${ }^{12} \mathrm{C}(\alpha, \gamma){ }^{16} \mathrm{O}$

General presentation of ${ }^{12} \mathrm{C}(\alpha, \gamma)^{16} \mathrm{O}$

- Determines the ${ }^{12} \mathrm{C} /{ }^{16} \mathrm{O}$ ratio

- Cross section needed near $\mathrm{E}_{\mathrm{cm}}=300 \mathrm{keV}$ (barrier $\sim 2.5 \mathrm{MeV}$ )

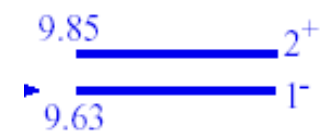

$\rightarrow$ cannot be measured in the Gamow peak

-1 ${ }^{-}$and $2^{+}$subthreshold states

$\rightarrow$ extrapolation difficult

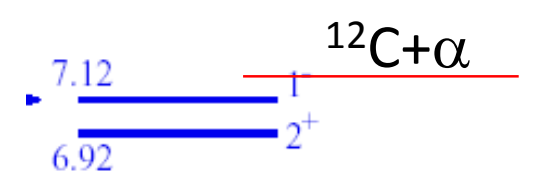

-E1 and E2 important (E1 forbidden when T=0)

- Interferences between $1_{1}^{-}, 1_{2}^{-}$and between $2^{+}{ }_{1}, 2^{+}{ }_{2}$

- Capture to gs dominant but also cascade transitions

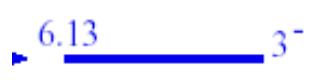

${ }^{16} \mathrm{O}$ 


\section{The R-matrix method}

Many experiments

- Direct ${ }^{12} \mathrm{C}(\alpha, \gamma){ }^{16} \mathrm{O}$ (angular distributions are necessary: E1 and E2)

- Indirect: spectroscopy of $1^{-}$and $2^{+}{ }_{1}$ subthreshold states

- Constraints

- $\alpha+{ }^{12} \mathrm{C}$ phase shifts $\left(1^{-} \rightarrow \mathrm{E} 1,2^{+} \rightarrow \mathrm{E} 2\right)$

- E1: ${ }^{16} \mathrm{~N}$ beta decay

(Azuma et al, Phys. Rev. C50 (1994) 1194) probes $\mathrm{J}=1^{-} \rightarrow \mathrm{E} 1$

- E2: ???

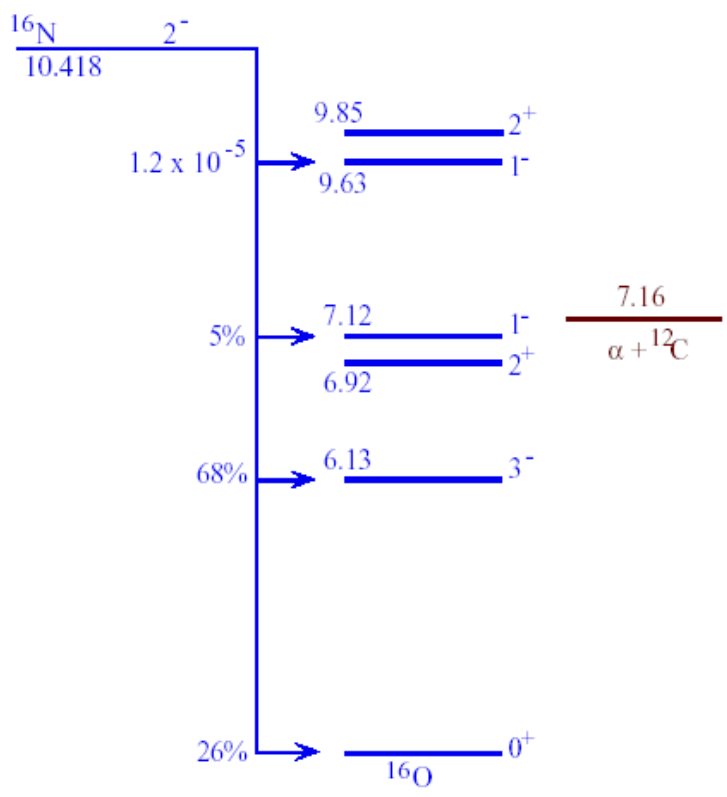




\section{The R-matrix method}

\section{Current situation}

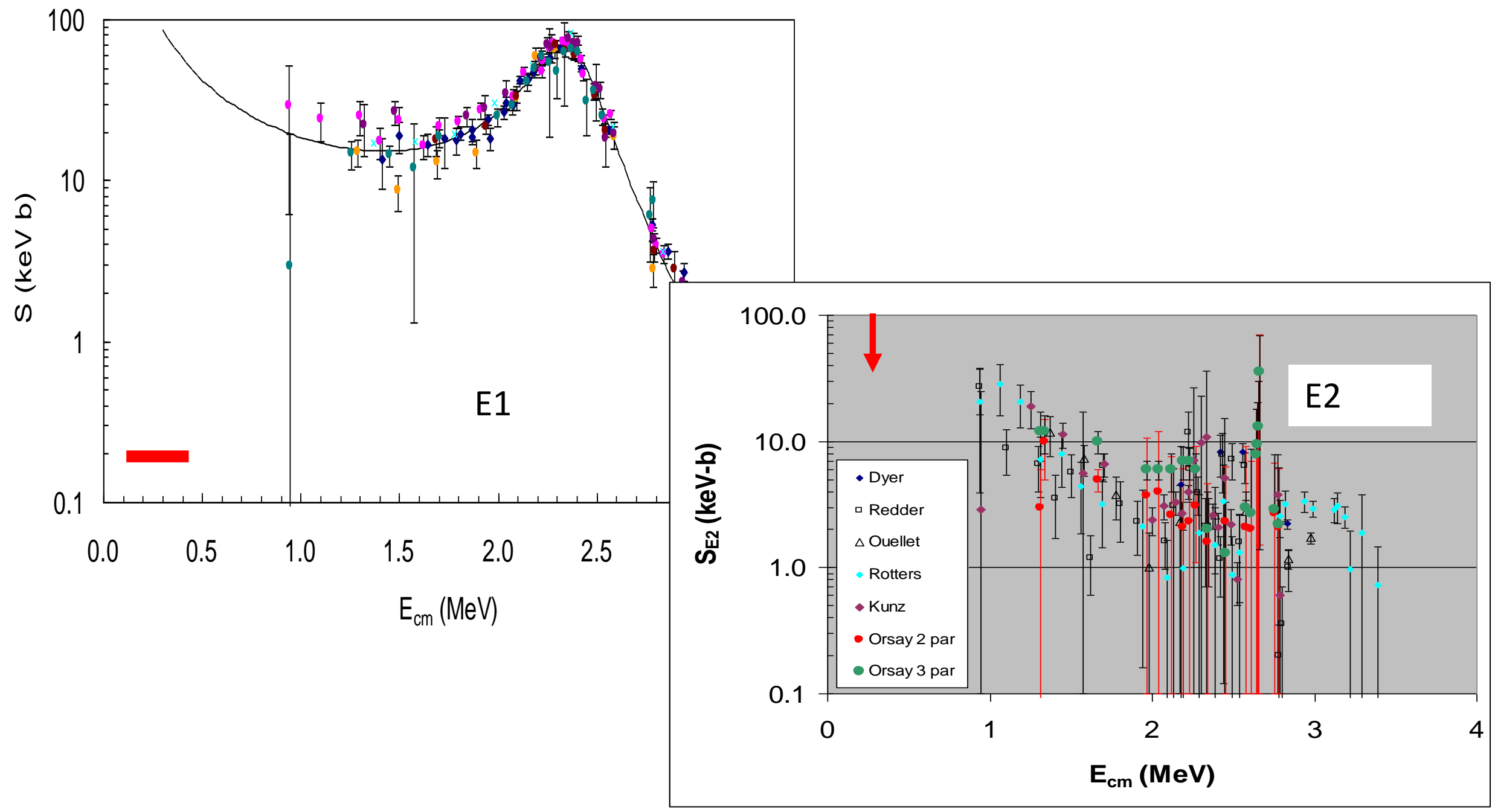




\section{The R-matrix method}

$S(300 \mathrm{keV})$ : current situation for E1

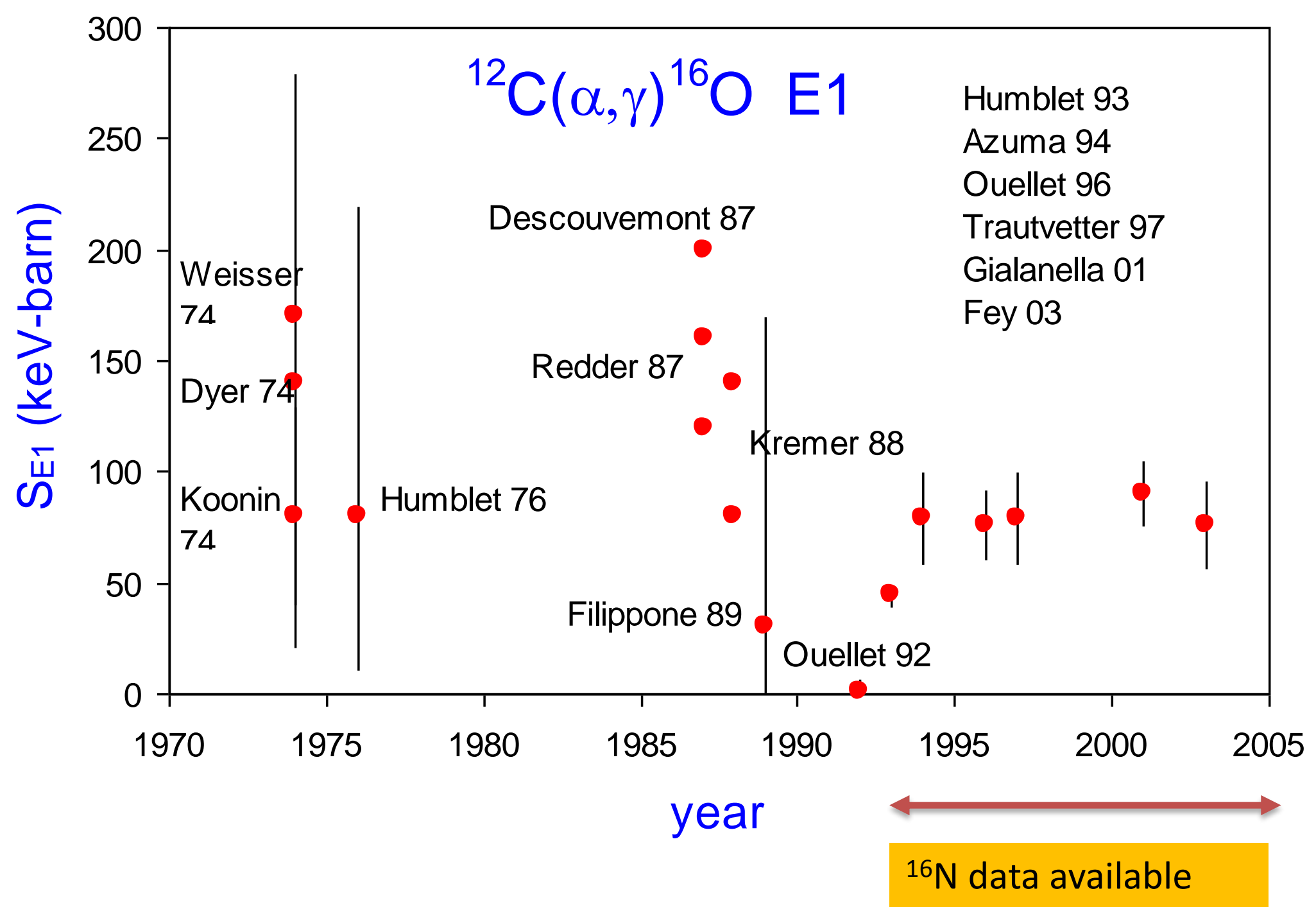




\section{The R-matrix method}

$S(300 \mathrm{keV})$ : current situation for E2

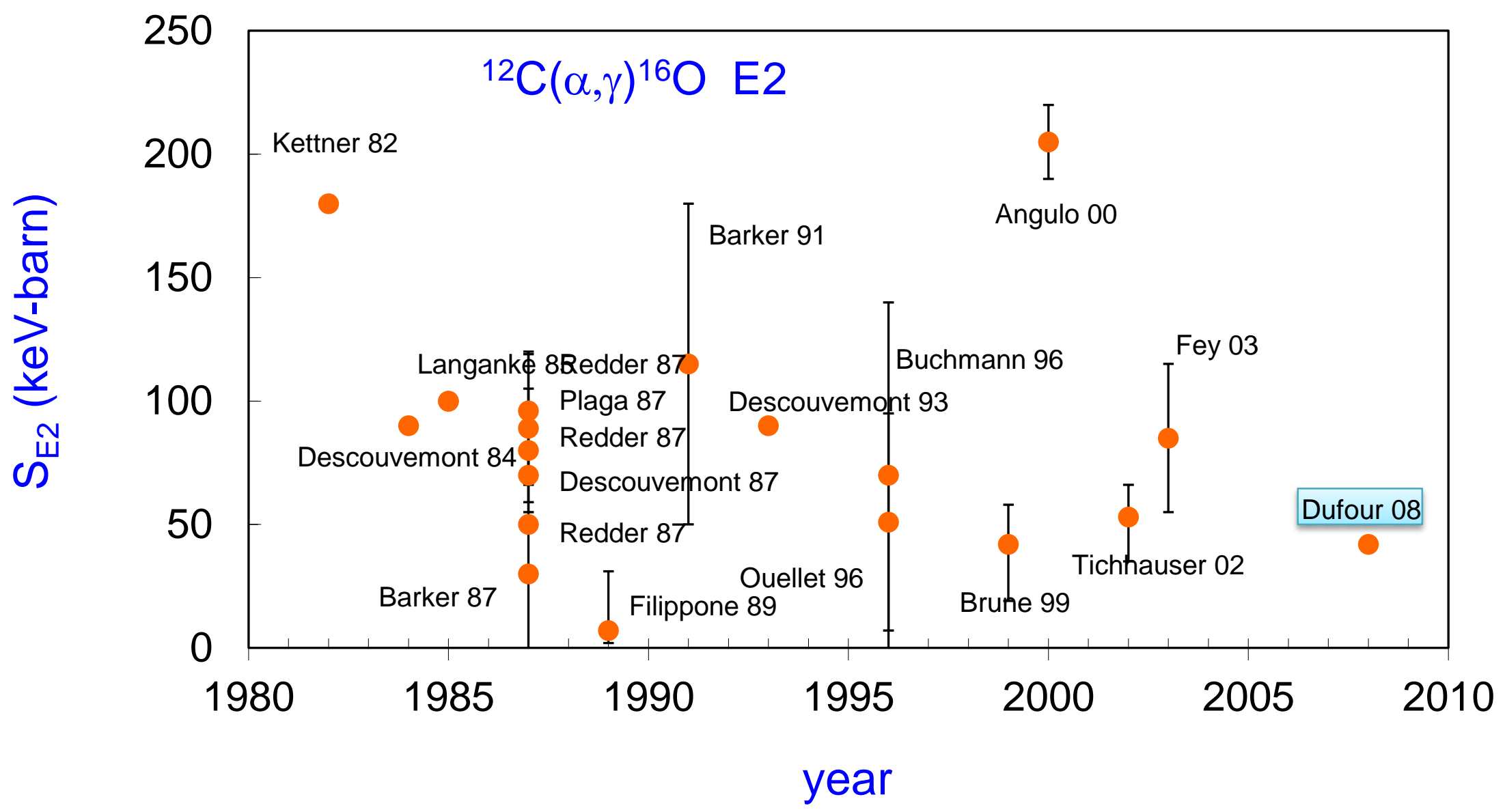


9. Microscopic models 


\section{Microscopic models}

○ Goal: solution of the Schrödinger equation $H \Psi=E \Psi$

○ Hamiltonian: $H=\sum_{i} T_{i}+\sum_{j>i} V_{i j}$

$\mathrm{T}_{\mathrm{i}}=$ kinetic energy of nucleon $i$

$\mathrm{V}_{\mathrm{ij}}=$ nucleon-nucleon interaction

○ Cluster approximation $\Psi=\mathcal{A} \phi_{1} \phi_{2} g(\rho)$

with $\phi_{1}, \phi_{2}=$ internal wave functions (input, shell-model)

$g(\rho)=$ relative wave function (output)

$\mathcal{A}=$ antisymmetrization operator

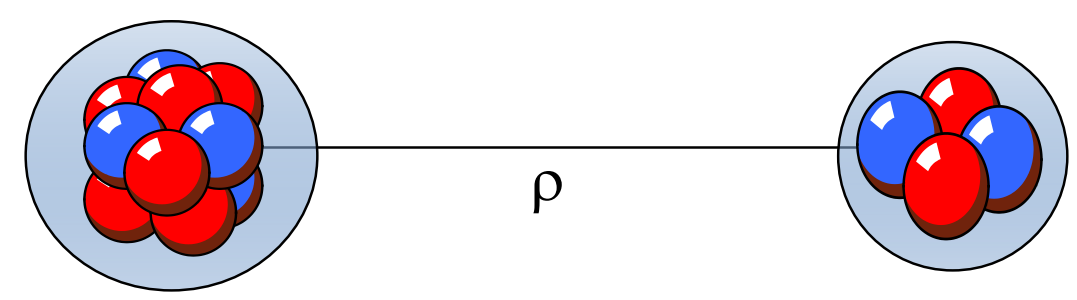

- Generator Coordinate Method (GCM): the radial function is expanded in Gaussians $\rightarrow$ Slater determinants (well adapted to numerical calculations)

o Microscopic R-matrix: extension of the standard R-matrix $\rightarrow$ reactions 


\section{Microscopic models}

Many applications: not only nuclear astrophysics

spectroscopy, exotic nuclei, elastic and inelastic scattering, etc.

Extensions:

- Multicluster calculations: $\rightarrow$ deformed nuclei (example: ${ }^{7} \mathrm{Be}+\mathrm{p}$ )

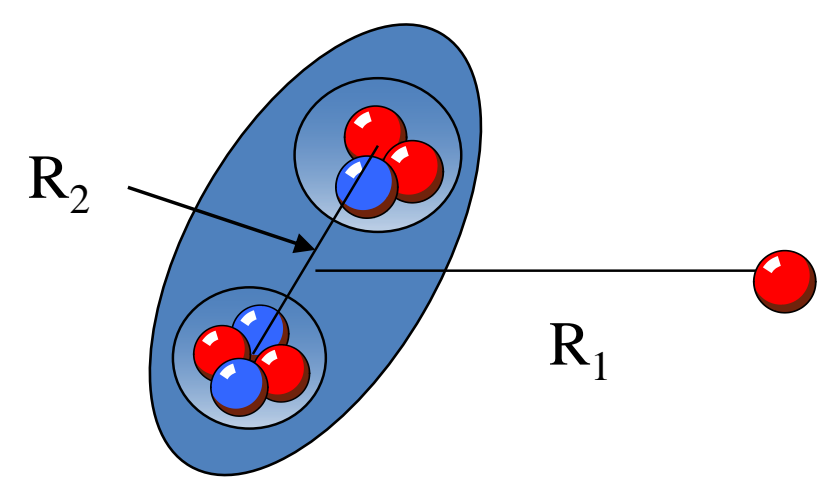

- Multichannel calculations: $\Psi=\mathcal{A} \phi_{1} \phi_{2} g(\rho)+\mathcal{A} \phi_{1}^{*} \phi_{2}^{*} g^{*}(\rho)+\cdots$

$\rightarrow$ better wave functions

$\rightarrow$ inelastic scattering, transfer

- Ab initio calculations: no cluster approximation

$\rightarrow$ very large computer times

$\rightarrow$ limited to light nuclei

$\rightarrow$ difficult for scattering (essentially limited to nucleon-nucleus) 


\section{Microscopic models}

\section{Example: ${ }^{7} \mathrm{Be}(\mathrm{p}, \gamma)^{8} \mathrm{~B}$}

- Important for the solar-neutrino problem

- Since 1995, many experiments:

- Direct (proton beam on a ${ }^{7} \mathrm{Be}$ target)

- Indirect (Coulomb break-up)

- Extrapolation to zero energy needs a theoretical model (energy dependence)

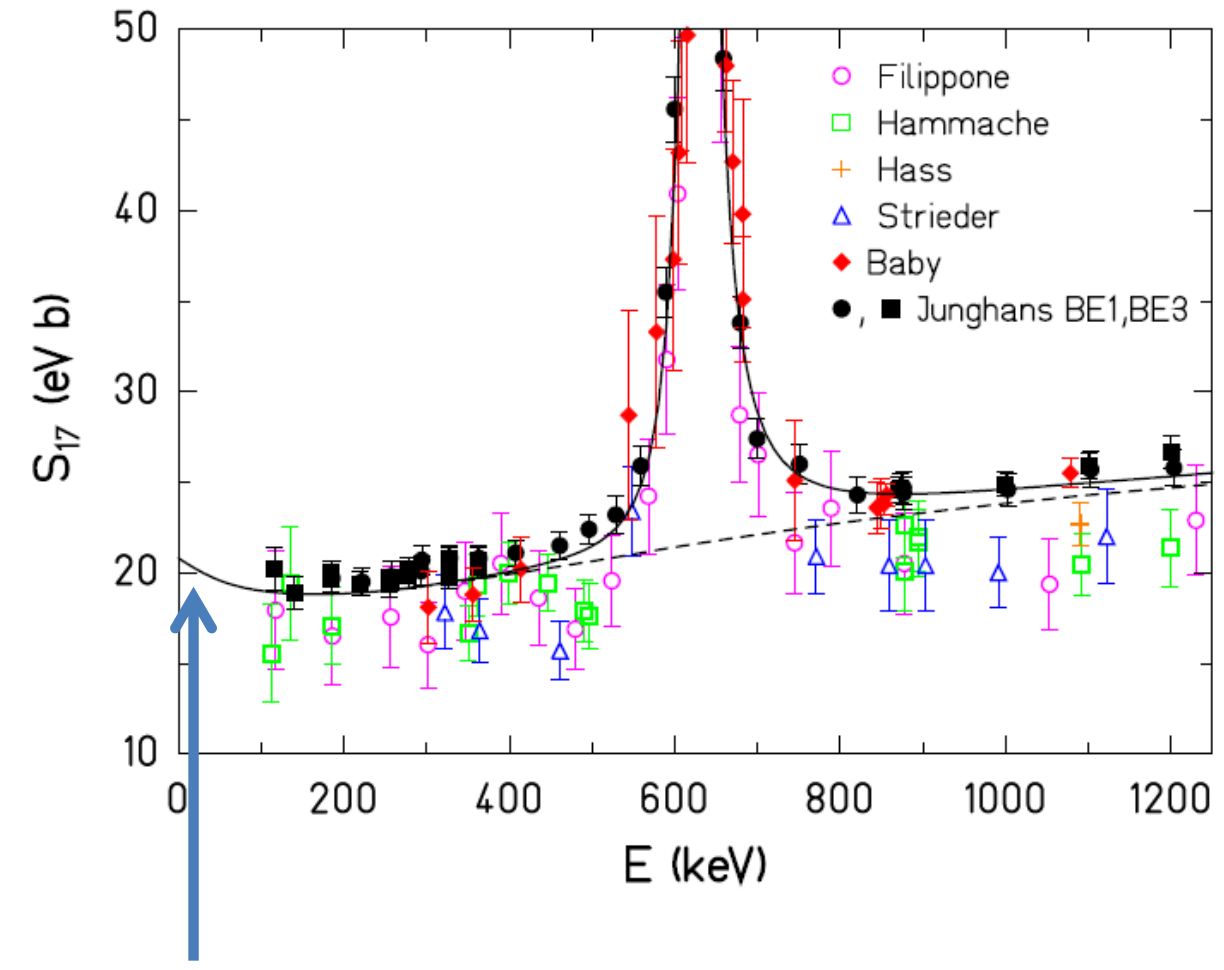

From E. Adelberger et al., Rev. Mod. Phys.83 (2011) 196 


\section{Microscopic models}

\section{Example: ${ }^{7} \mathrm{Be}(\mathrm{p}, \gamma)^{8} \mathrm{~B}$}

- Microscopic cluster calculations: 3-cluster calculations

- P. D. and D. Baye, Nucl. Phys. A567 (1994) 341

- P.D., Phys. Rev. C 70, 065802 (2004)

- Includes the deformation of ${ }^{7} \mathrm{Be}$ : cluster structure $\mathrm{a}+{ }^{3} \mathrm{He}$

- Includes rearrangement channels ${ }^{5} \mathrm{Li}+{ }^{3} \mathrm{He}$

- Can be applied to ${ }^{8} \mathrm{~B} /{ }^{8} \mathrm{Li}$ spectroscopy

- Can be applied to ${ }^{7} \mathrm{Be}(\mathrm{p}, \gamma)^{8} \mathrm{~B}$ and ${ }^{7} \mathrm{Li}(\mathrm{n}, \gamma)^{8} \mathrm{Li}$
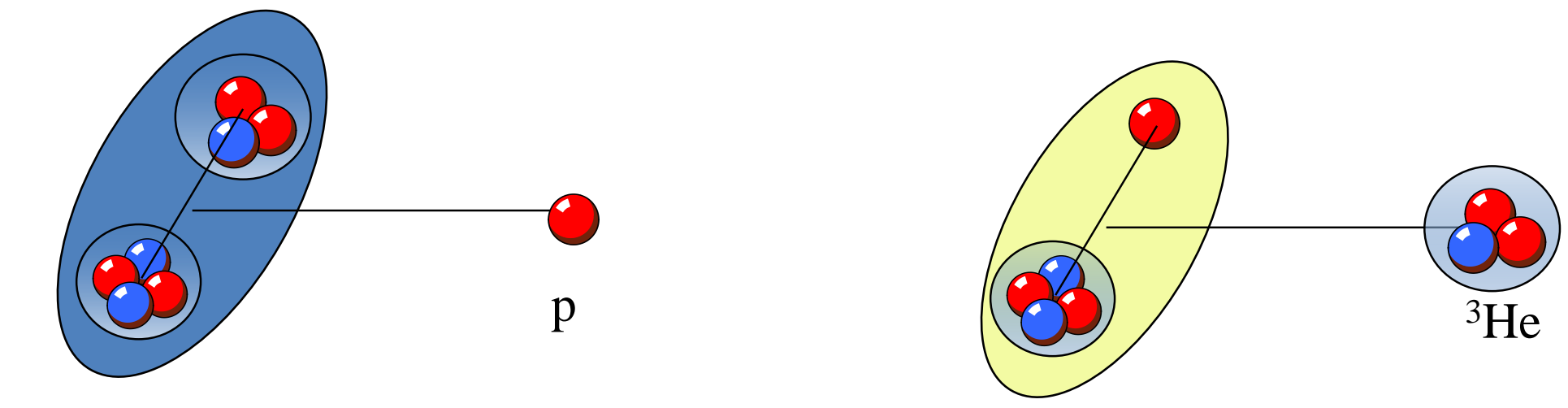

${ }^{7} \mathrm{Be}=\alpha+{ }^{3} \mathrm{He}$

${ }^{5} \mathrm{Li}=\alpha+\mathrm{p}$ 


\section{Microscopic models}

\section{Spectroscopy of ${ }^{8}$ B}

\begin{tabular}{|l|c|c|c|}
\hline & experiment & Volkov & Minnesota \\
\hline$\mu\left(2^{+}\right)\left(\mu_{\mathrm{N}}\right)$ & 1.03 & 1.48 & 1.52 \\
\hline $\mathrm{Q}\left(2^{+}\right)\left(\mathrm{e.fm}{ }^{2}\right)$ & $6.83 \pm 0.21$ & 6.6 & 6.0 \\
\hline $\mathrm{B}\left(\mathrm{M} 1,1^{+} \rightarrow 2^{+}\right)$(W.u. $)$ & $5.1 \pm 2.5$ & 3.4 & 3.8 \\
\hline
\end{tabular}

Channel components in the ${ }^{8} \mathrm{~B}$ ground state

\begin{tabular}{|l|l|}
\hline${ }^{7} \mathrm{Be}\left(3 / 2^{-}\right)+p$ & $47 \%$ \\
\hline${ }^{7} \mathrm{Be}\left(1 / 2^{-}\right)+\mathrm{p}$ & $9 \%$ \\
\hline${ }^{5} \mathrm{Li}\left(3 / 2^{-}\right)+{ }^{3} \mathrm{He}$ & $34 \%$ \\
\hline${ }^{5} \mathrm{Li}\left(1 / 2-2^{-}\right)+{ }^{3} \mathrm{He}$ & $3 \%$ \\
\hline
\end{tabular}

$\Rightarrow$ Important role of the $5+3$ configuration 


\section{Microscopic models}

\section{${ }^{7} \mathrm{Be}(\mathrm{p}, \gamma)^{8} \mathrm{~B}$ S factor}

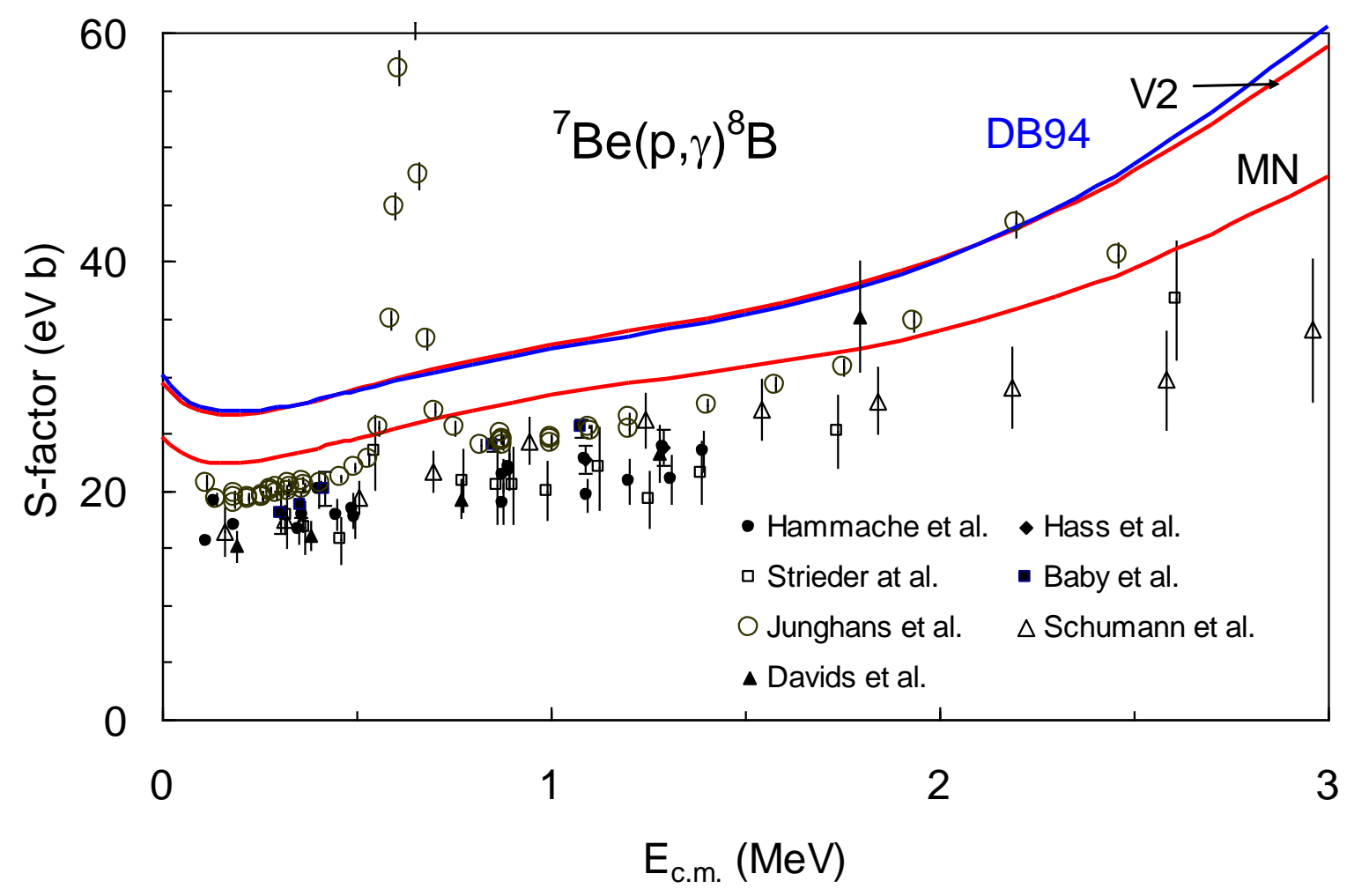

- Low energies $(E<100 \mathrm{keV})$ : energy dependence given by the Coulomb functions

- $2 \mathrm{NN}$ interactions (MN, V2): $\rightarrow$ the sensitivity can be evaluated

- Overestimation: due to the ${ }^{8} \mathrm{~B}$ ground state (cluster approximation) 


\section{Microscopic models}

\section{Cluster models}

- In general a good approximation, but do not allow the use of realistic NN interactions

- Example: $\alpha$ particle described by 4 0s orbitals

$\rightarrow$ intrinsic spin $=0$

$\rightarrow$ no spin-orbit, no tensor force, no 3-body force

$\rightarrow$ these terms are simulated by (central) NN interactions

\section{Ab initio models}

- No cluster approximation

- Use of realistic NN interactions (fitted on deuteron, NN phase shifts, etc.)

- Application: $d+d$ systems ${ }^{2} \mathrm{H}(d, \gamma){ }^{4} \mathrm{He},{ }^{2} \mathrm{H}(\mathrm{d}, \mathrm{p}){ }^{3} \mathrm{H},{ }^{2} \mathrm{H}(\mathrm{d}, \mathrm{n})^{3} \mathrm{He}$ two physics issues

- Analysis of the $d+d$ S factors (Big-Bang nucleosynthesis)

- Role of the tensor force in ${ }^{2} \mathrm{H}(\mathrm{d}, \gamma)^{4} \mathrm{He}$ 


\section{Microscopic models}

${ }^{2} \mathrm{H}(\mathrm{d}, \gamma)^{4} \mathrm{He} \mathrm{S}$ factor

- Ground state of ${ }^{4} \mathrm{He}=0+$

- $\mathrm{E} 1$ forbidden $\rightarrow$ main multipole is $\mathrm{E} 2 \rightarrow 2^{+}$to $0^{+}$transition $\rightarrow \mathrm{d}$ wave as initial state

- Experiment shows a plateau below $0.1 \mathrm{MeV}$ : typical of an $s$ wave

- Interpretation : the ${ }^{4} \mathrm{He}$ ground state contains an admixture of $\mathrm{d}$ wave final $0^{+}$state: $\Psi^{0+}=\Psi^{0+}(L=0, S=0)+\Psi^{0+}(L=2, S=2)=\left|0^{+}, 0>+\right| 0^{+}, 2>$ initial $2^{+}$state: $\Psi^{2+}=\Psi^{2+}(L=2, S=0)+\Psi^{2+}(L=0, S=2)=\left|2^{+}, 0>+\right| 2^{+}, 2>$

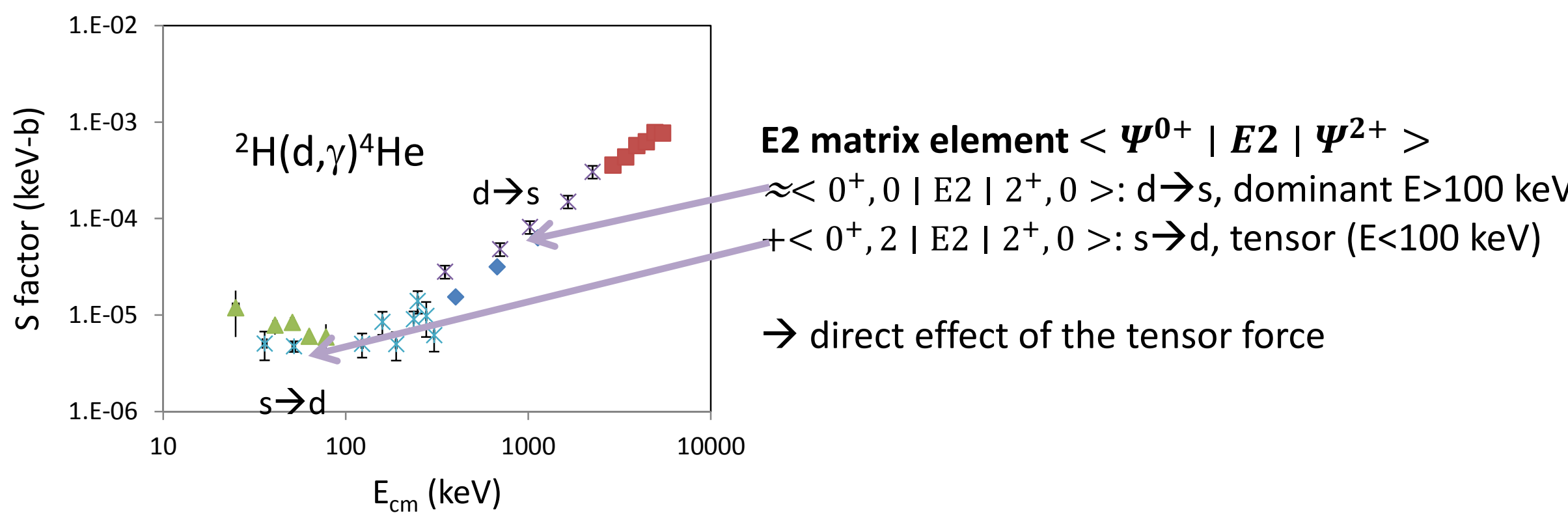




\section{Microscopic models}

Application: $d+d$ systems

- Collaboration Niigata (K. Arai, S. Aoyama, Y. Suzuki)-Brussels (D. Baye, P.D.) Phys. Rev. Lett. 107 (2011) 132502

- Mixing of $d+d,{ }^{3} \mathrm{H}+p,{ }^{3} \mathrm{He}+n$ configurations

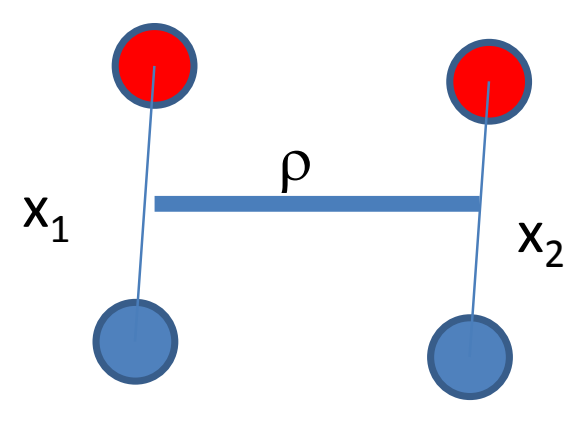

$d+d$

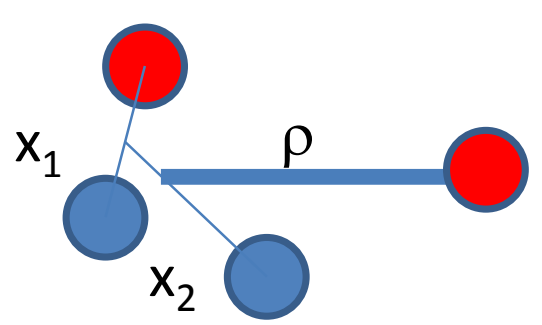

$3 \mathrm{H}+\mathrm{p}, 3 \mathrm{He}+\mathrm{n}$

- The total wave function is written as an expansion over a gaussian basis

- Superposition of several angular momenta

- 4-body problem (in the cluster approximation we would have: $x_{1}=x_{2}=0$ ) 


\section{Microscopic models}

We use 3 NN interactions:

- Realistic: Argonne AV8', G3RS

- Effective: Minnesota MN

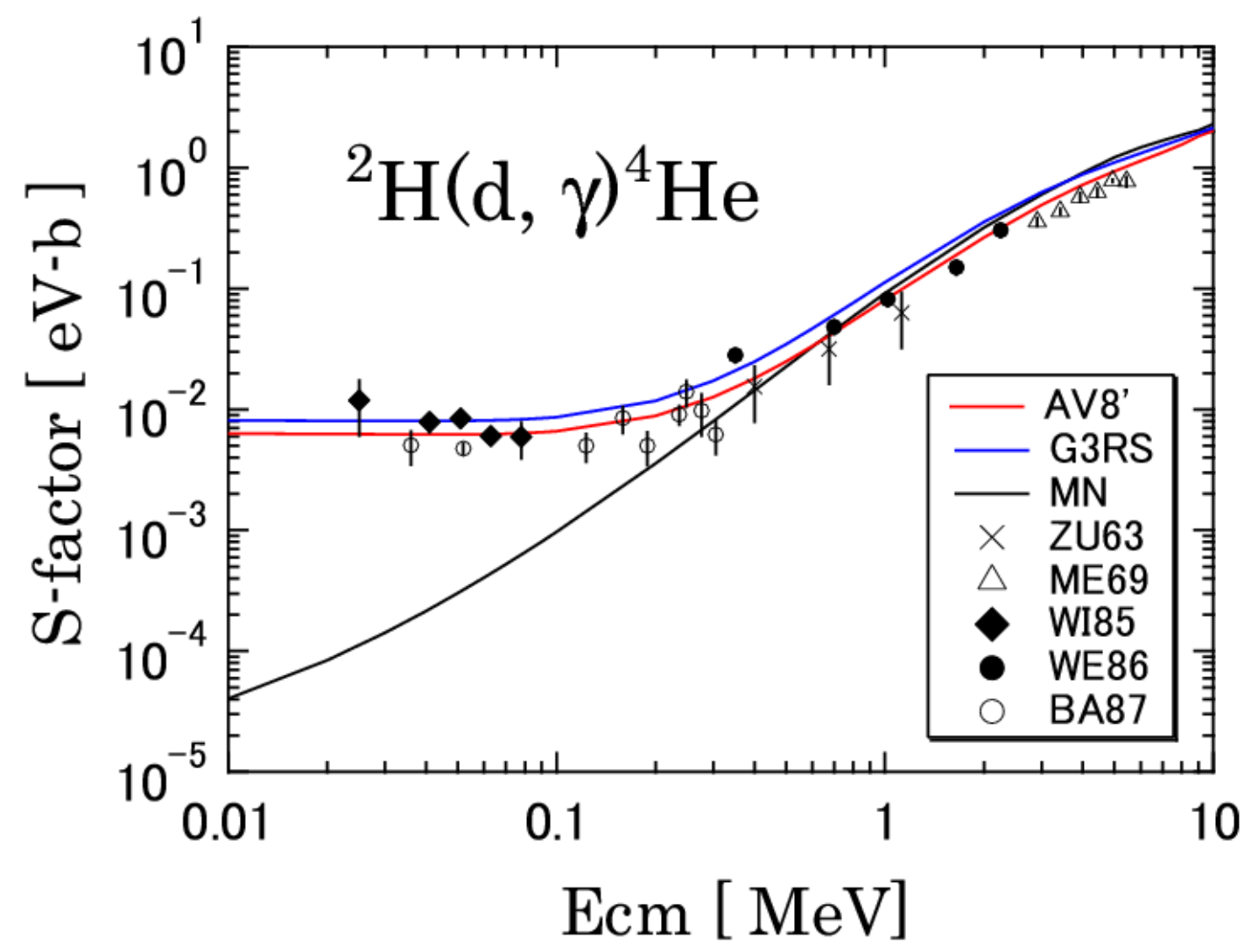

- No parameter

- MN does not reproduce the plateau (no tensor force)

- D wave component in ${ }^{4} \mathrm{He}$ : 13.8\% (AV8') $11.2 \%$ (G3RS) 


\section{Microscopic models}

Transfer reactions ${ }^{2} \mathrm{H}(\mathrm{d}, \mathrm{p})^{3} \mathrm{H},{ }^{2} \mathrm{H}(\mathrm{d}, \mathrm{n})^{3} \mathrm{He}$

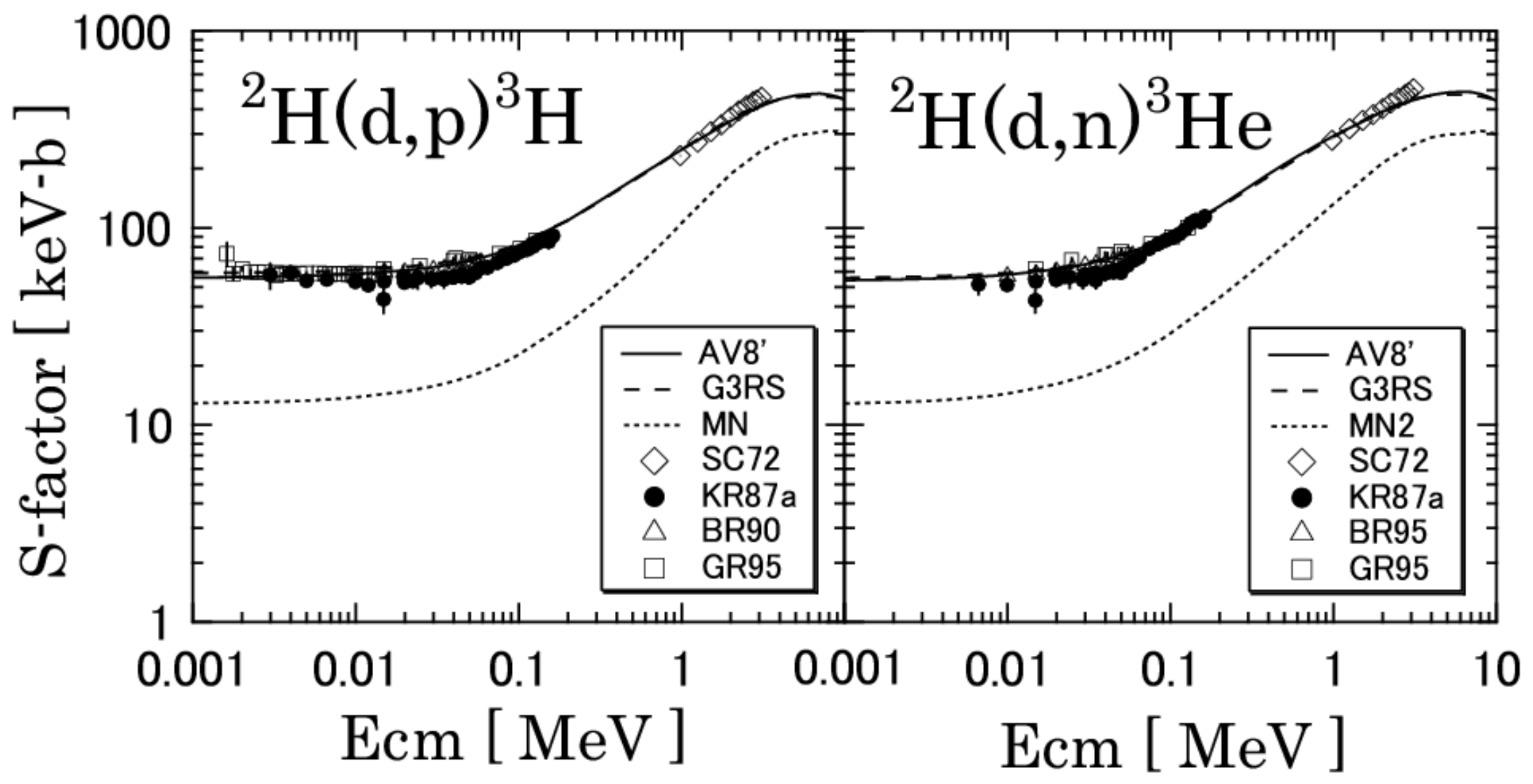




\section{Conclusions}

\section{Needs for nuclear astrophysics:}

- low energy cross sections

- resonance parameters

Experiment: direct and indirect approaches

Theory: various techniques

- fitting procedures ( $R$ matrix) $\rightarrow$ extrapolation

- non-microscopic models: potential, DWBA, etc.

- microscopic models:

cluster: developed since 1960's, applied to NA since 1980's

$>$ ab initio: problems with scattering states, resonances $\rightarrow$ limited at the moment

- Current challenges: new data on ${ }^{3} \mathrm{He}(\alpha, \gamma)^{7} \mathrm{Be}$, triple $\alpha$ process, ${ }^{12} \mathrm{C}(\alpha, \gamma){ }^{16} \mathrm{O}$, etc. $\mathrm{D}(\mathrm{d}, \gamma)^{4} \mathrm{He}: 4$ nucleons $\rightarrow 4$ clusters 